

\section{MAIL ORDERS}

Mail Orders (in English) for all NIST SRMs/RMs should be directed to:

Standard Reference Materials Program

National Institute of Standards and Technology 100 Bureau Drive, Stop 2322

Gaithersburg, MD 20899-2322

USA

Telephone: (301) 975-6776

Fax: (301) 948-3730

E-Mail: srminfo@nist.gov

www.nist.gov/srm

Each purchase order should give the number of units, SRM number, and name of each reference material requested.

\section{Example:}

1 unit, SRM 930e Glass Filters for Spectrophotometry

The following information must be included with each order:

- name of customer

- shipping address

billing address

- telephone number

fax number

- purchase order number

- a customer identification number, i.e., a social security number (SSN) for consumer customers, tax identification number (TIN) for commercial customers, or agency code (ALC) for U.S. Government customers

Note: NIST SRMs/RMs are only distributed in the units of issue listed in this catalog and its supplement (price list). All purchase orders must be in English. 


\section{Standard Reference Materials Catalog}

February 2002

Joylene W.L. Thomas, Editor

Yvonne A. Branden, Co-Editor

Robbin D. Howard, Co-Editor

Standard Reference Materials Program

Technology Services

National Institute of Standards and Technology

Gaithersburg, MD 20899-2320

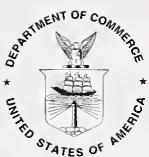

U.S. Department of Commerce

Donald L. Evans, Secretary

Technology Administration

Phillip J. Bond

Under Secretary of Commerce for Technology

National Institute of Standards and Technology Arden L. Bement, Jr., Director 
Certain commercial entities, equipment, or materials may be identified in this document in order to describe an experimental procedure or concept adequately. Such identification is not intended to imply recommendation or endorsement by the National Institute of Standards and Technology, nor is it intended to imply that the entities, materials, or equipment are necessarily the best available for the purpose.

National Institute of Standards and Technology Special Publication 260

Supersedes NIST SP 260, 1998-1999

100 pages (February 2002)

CODEN: NSPUE2

\section{U.S. GOVERNMENT PRINTING OFFICE}

WASHINGTON: 2002

For sale by the Superintendent of Documents, U.S. Government Printing Office Internet: bookstore.gpo.gov

Phone: (202) 512-1800

Fax: (202) 512-2250

Mail: Stop SSOP, Washington, DC 20402-0001 

IST Standard Reference Materials ${ }^{\oplus}\left(\mathrm{SRMs}^{\oplus}\right)$ are used by industry, government, and academia to ensure the highest quality measurements. This catalog lists over 1300 individual reference materials produced and sold by NIST, each with carefully assigned values for chemical composition and physical properties.

SRMs find use in calibrating instruments and in assuring the long-term integrity of quality assurance programs. They are also key mechanisms for verifying important measurement results and in developing new measurement methods. SRMs provide users with tools to assist in establishing traceability of measurement results to NIST.

Each SRM comes carefully packaged with documentation containing assigned values with stated uncertainties and a material safety data sheet, if applicable. Details on use, stability, and NIST analytical methods are also included.

For further information and prices, contact us at:

Telephone: (301) 975-6776

Fax: (301) 948-3730

E-mail: srminfo@nist.gov

www.nist.gov/srm 


\section{Engineering Materials}

$1 \mathrm{SIZING}$

1 Particle Size

2 Cement Turbidity and Fineness

2 Surface Area of Powders

2 Particle Count Materials

2 SURFACE FINISH

2 Abrasive Wear

3 Surface Roughness

3 FIRE RESEARCH

3 Surface Flammability

4 Smoke Density Chamber

4 Smoke Toxicity

4 Flooring Radiant Panel

5 NONDESTRUCTIVE

EVALUATION

5 Artificial Flaw for Eddy Current NDE

5 PERFORMANCE

ENGINEERING MATERIALS

5 Fracture Toughness of Steels (Charpy V-Notch Test Blocks)

5 Rockwell Hardness

6 Microindentation Hardness (Knoop and Vickers Test Blocks)

6 Coordinate Measuring Machine (CMM) Probe Performance

6 Tape Adhesion Testing

7 Bleached Kraft Pulps

7 Secondary Ferrite Number (FN) Materials

7 Fracture Toughness of Ceramics

7 Magnetic Moment Standards

\section{Food \& Agriculture}

9 Trace Elements in Food and Dairy Products

9 Wheat Hardness

10 Nutrition Composition

11 Trace Elements in

Botanicals

11 Fertilizers

11 Whole Biomass Feedstock

\section{Health \& Clinical}

13 Pure, Crystalline Standards

13 Biological Buffer Systems

14 Human Serum

15 Bovine Serum

15 Ethanol Solutions

16 DNA Profiling

16 Biomaterials

17 Drugs of Abuse in Urine

17 Toxic Substances in Urine

17 Miscellaneous Health-Related Standards

\section{Environmental}

19 ORGANICS

19 Gas Chromatography/Mass Spectrometry (GC/MS) and Characterizing Liquid Chromatography (LC) System Performance

20 Organic Contaminant Calibration Solutions

21 Organic Contaminants in Natural Matrix Materials

22 EPA: Organic Compounds Related to Water Analysis

23 INORGANICS

23 Metal Constituents in Natural Matrices: Air Particulate, Indoor Dust, Sediment, Sludge, Soil, and Water

24 Carbon Modified Silica

24 Used Auto Catalysts

24 Primary Gas Mixtures

28 FOSSIL FUELS

28 Metal Constituents in Fossil Fuels

28 High Purity Liquids for Fuel Rating

28 Trace Elements in Coals and Coke

29 Alcohols and Ethers [Oxygenates] in Reference Fuels

30 Sulfur in Fossil Fuels

31 Moisture in Oils and Alcohols

32 GEOLOGICAL MATERIALS

AND ORES

32 Ores

33 Ore Bioleaching Substrate

33 Chinese Ores

33 Clays

34 Rocks and Minerals

34 Refractories

35 MICROANALYSIS

35 Metals

35 Synthetic Glasses

35 Thin Film for Transmission Electron Microscope
36 Metallo-Organic Compounds

37 Lubricating Base Oils

37 Catalyst Characterization Material

37 Wear-Metals in Oil

\section{High Purity Materials}

39 Elemental Composition in High Purity Metals

40 Fine Gold Standards

40 Stoichiometric Standards

41 Microchemistry

42 Spectrometric Single Element Solutions

44 Anion Chromatography Solutions

44 Stable Isotopic Materials

45 Light Stable Isotopic Materials

\section{Industrial Materials}

47 FERROUS METALS

47 Steels

47 Plain Carbon Steels

48 Low Alloy Steels

50 Special Low Alloy Steels

50 High Alloy Steels

51 Stainless Steels

52 Specialty Steels

52 Tool Steels

53 Cast Steels, White Cast Irons, and Ductile Irons

53 Steelmaking Alloys

54 Cast Irons

55 High Temperature Alloys

55 Gases in Metals: Iron and Steel

56 NONFERROUS METALS

56 Aluminum Base Alloys

56 Cobalt Base Alloys

57 Copper "Benchmark"

57 Copper Base Alloys

58 Lead Base Alloys

58 Lead Base Materials

59 Nickel Oxides

59 Nickel Base Alloys

59 Trace Elements in Nickel Base Superalloys

60 Tin Base Alloys

60 Titanium Base Alloys

60 Hydrogen in Titanium

60 Zirconium Base Alloys

61 Zinc Base Alloys

61 CERAMICS AND GLASSES 61 Carbides

61 Cemented Tungsten Carbides

62 Glasses

62 Trace Elements

63 CEMENTS

63 Portland Cements

63 Portland Cement Clinkers 


\section{Physical Properties}

65 ION ACTIVITY

$65 \mathrm{pH}$ Calibration

66 Biological Buffer Systems

66 pD Calibration

66 Ion-Selective Electrode

Calibration

67 Electrolytic Conductivity

67 Positive Electrophoretic Mobility

68 POLYMERIC PROPERTIES 68 Molar Mass/Molecular Weight

69 Melt Flow Rate

69 Viscosity

69 Biomaterials

70 THERMODYNAMIC

PROPERTIES

70 Calorimetry - Combustion

70 Calorimetry - Solution

70 Enthalpy and Heat Capacity

71 Differential Scanning Calorimetry

71 Differential Thermal Analysis

71 Defining Fixed Points, International Temperature Scale of 1990 , ITS-90

72 Reference Points

72 Freezing Point, Melting Point, and Triple Point Cells

72 Thermal Expansion of Metal and Glass

72 Thermal Resistance of Glass, Silica, and Polystyrene

73 Vapor Pressure of Metals

73 Thermal Conductivity of Graphite and Iron

73 Laboratory Thermometer

73 Thermocouple Material, Platinum

74 OPTICAL PROPERTIES

74 Molecular Transmittance and Absorbance

75 Transmittance Wavelength Standards

75 Fluorescence

75 Specular Spectral Reflectance

75 Near Infrared Reflectance Wavelength Standard

76 Optical Rotation

76 Liquid Refractive Index

76 X-ray and Photographic Imaging

77 ELECTRICAL PROPERTIES

77 Electrical Resistivity and

Conductivity of Electrolytic Iron and Graphite

77 Electrical Resistivity and Conductivity of Silicon

OPTOELECTRONICS
78 METROLOGY

78 Optical Microscope Linewidth Measurement

79 Scanning Electron Microscope (SEM)

79 Depth Profiling

79 Solder Thickness for X-ray Fluorescense

80 Coating Thickness

80 Ellipsometry

81 oxygen Concentration in Silicon

81 Superconducting Critical Current

81 CERAMICS AND GLASSES

81 Chemical Resistance [Durability]

81 Electrical Properties

82 Viscosity

82 Viscosity Fixpoints

82 Relative Stress Optical Coefficient

82 Density (glass \& liquid)

83 Glass Liquidus Temperature

83 X-RAY SPECTROMETRY

83 X-ray Diffraction

83 X-ray Stage Calibration

\section{Radioactivity}

85 Radioactive Solutions

87 Radioactive Point Sources

87 Radiopharmaceuticals

88 Radon Emanation

88 Beryllium Isotopic Ratio Standard

88 Carbon-14 Dating

89 Natural Matrix Materials

89 Neutron Density Monitor Wire

89 Fission Track Glass

\section{Industrial Hygiene}

91 Materials on Filter Media

91 Trace Constituent Elements in Blank Filters

91 Respirable Silica

92 Lead in Paint, Dust, and Soil

93 Asbestos
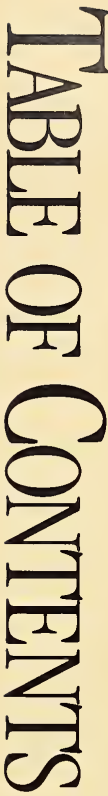


\section{ENGINEERTG MATERIALS}

1 Stzing

2 Surface Finish

3 Fire Research

5 Nondestructlve Evaluation

5 Performance Engineering Materials

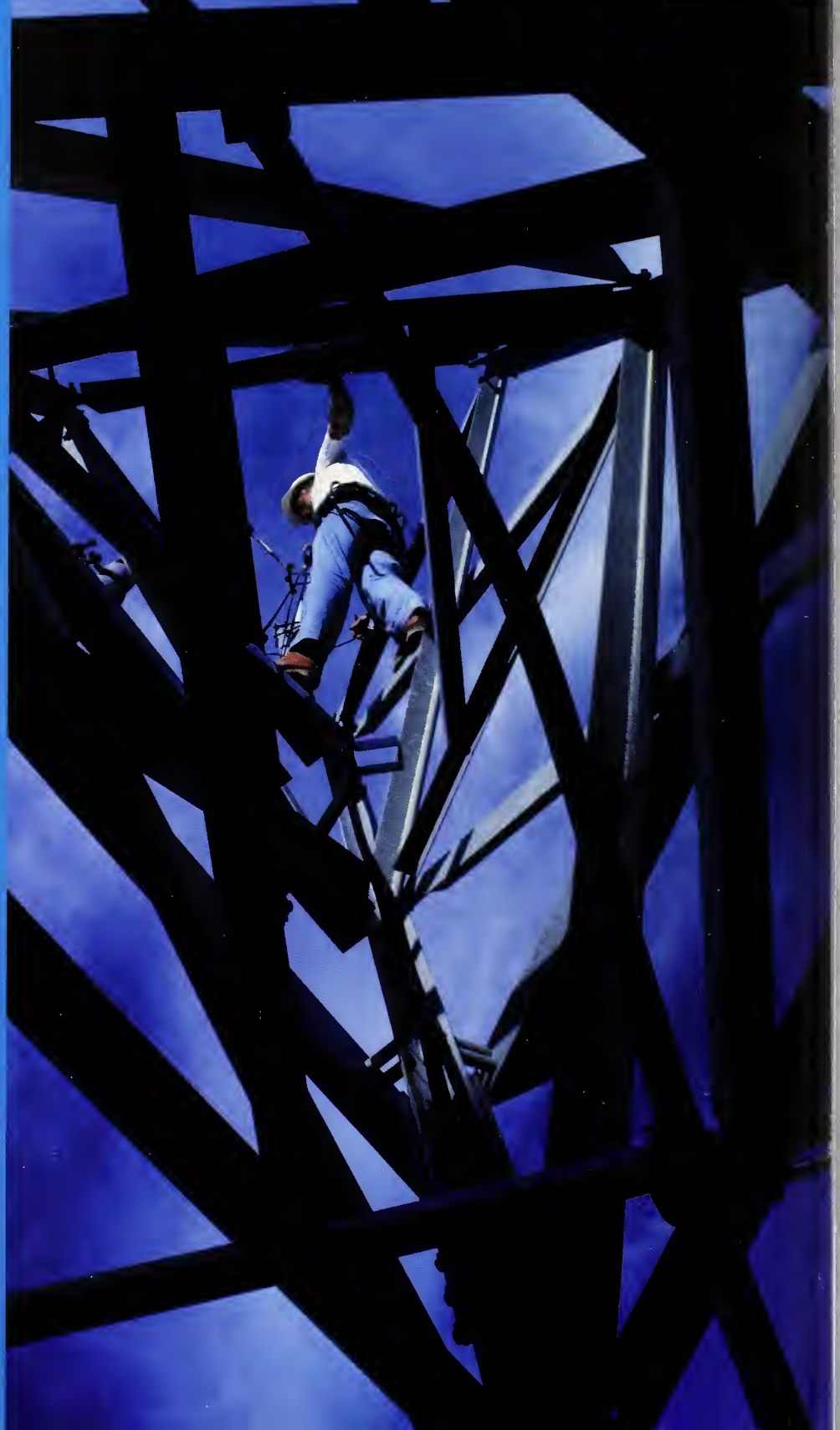




\section{S I Z I N G}

\section{Particle Size}

These SRMs are used for particle size measuring instruments, including light scattering, electrical zone flow-through counters, optical and scanning electron microscopes, sedimentation systems, and wire cloth sieving devices.

\section{SRM}

Glass Beads, Soda Lime

1003d (In Prep)

1004b

$1017 \mathrm{~b}$

$1018 b$

$1019 b$

Sand

RM 8010

(No. 30 to No. 325)

$3 \times 150 g$

Silicon Nitride (equiaxed)

659

$0.2 \mu \mathrm{m}$ to $10 \mu \mathrm{m}$

$5 \times 2.5 g$

43

70

87

200

\section{Unit Size (g)}
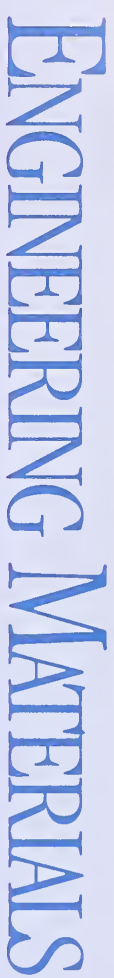

\section{Tungsten Carbide/Cobalt (spheroidal)}

$1984 \quad 9 \mu \mathrm{m}$ to $30 \mu \mathrm{m} \quad 14$

1985

$18 \mu \mathrm{m}$ to $55 \mu \mathrm{m}$

14

\section{Polystyrene Spheres}

Unit Size: $5 \mathrm{~mL}$ vial (unless otherwise noted)

$\begin{array}{ll}1690\left(\mathrm{O} .5 \% \text { in } \mathrm{H}_{2} \mathrm{O}\right) & 0.895 \mu \mathrm{m} \\ 1691\left(0.5 \% \text { in } \mathrm{H}_{2} \mathrm{O}\right) & 0.269 \mu \mathrm{m} \\ 1692\left(0.25 \% \text { in } \mathrm{H}_{2} \mathrm{O}\right) & 2.982 \mu \mathrm{m} \\ 1960 *\left(0.4 \% \text { in } \mathrm{H}_{2} \mathrm{O}\right) & 9.89 \mu \mathrm{m} \\ 1961 *\left(0.5 \% \text { in } \mathrm{H}_{2} \mathrm{O}\right) & 29.64 \mu \mathrm{m} \\ 1963\left(0.5 \% \text { in } \mathrm{H}_{2} \mathrm{O}\right) & 0.1007 \mu \mathrm{m} \\ 1965 \text { (Slide Mounted: } 1 \text { slide) } & 9.94 \mu \mathrm{m} \text { (hexagonal array) } \\ & 9.89 \mu \mathrm{m} \text { (unordered clusters) }\end{array}$

*Developed in cooperation with NASA 


\section{Cement Turbidity and Fineness}

This SRM is suitable for use with ASTM C 430-92, C 115-93, and C 204-92.

\begin{tabular}{|c|c|c|c|c|}
\hline SRM & Description & Properties Certified & Value & Unit Size \\
\hline \multirow[t]{3}{*}{$114 p$} & Portland Cement & Sieve Residue (45 $\mu \mathrm{m}$ (No. 325) Sieve) & $8.24 \%$ & 20 pouches $\times 10 \mathrm{~g}$ \\
\hline & & $\begin{array}{l}\text { Specific Surface Area } \\
\text { (Wagner Turbidimeter) }\end{array}$ & $2086 \mathrm{~cm}^{2} \cdot \mathrm{g}^{1}$ & \\
\hline & & $\begin{array}{l}\text { Specific Surface Area } \\
\text { (Blaine Air Permeability) }\end{array}$ & $3774 \mathrm{~cm}^{2} \cdot \mathrm{g}^{-1}$ & \\
\hline
\end{tabular}

Surface Area of Powders (Brunauer, Emmett, and Teller Method)

$\begin{array}{ll}\text { SRM } & \text { Description } \\ 1899 & \text { Silicon Nitride } \\ 1900 & \text { Silicon Nitride }\end{array}$

\section{Particle Count Materials}

These SRMs are suitable for use with particle sizing instrumentation, including optical counters, in accordance with National Fluid Power Association (NFPA) T2.9.6 R2-1998 and ISO/DIS 11171.

$\begin{array}{llll}\text { SRM } & \text { Description } & \text { Particle Concentration } & \text { Unit Size } \\ 2806 & \text { Medium Test Dust in Hydraulic Fluid } & 2.8 \mathrm{mg} / \mathrm{L} & 400 \mathrm{~mL} \\ \text { RM } 8631 & \text { Medium Test Dust } & 1 \mu \mathrm{m} \text { to } 50 \mu \mathrm{m} & 20 \mathrm{~g} \\ \text { RM } 8632 & \text { Ultrafine Test Dust } & 1 \mu \mathrm{m} \text { to } 20 \mu \mathrm{m} & 20 \mathrm{~g}\end{array}$

\section{S UR FA C E F I N I S H}

\section{Abrasive Wear}

This SRM is suitable for use with ASTM G 65, Procedure A.

SRM

Description

1857
D-2 Tool Steel

\begin{tabular}{|c|c|}
\hline \multicolumn{2}{|c|}{ Surface Area $\left(\mathrm{m}^{2} / \mathrm{g}\right)$} \\
\hline Multi-point & Single Point \\
\hline 10.67 & 10.52 \\
\hline 2.85 & 2.79 \\
\hline
\end{tabular}

Unit Size (g)

4

4 


\section{Surface Roughness}

Unit Size: $25 \mathrm{~mm} \times 34 \mathrm{~mm} \times 12 \mathrm{~mm}$

These SRMs are used for calibrating stylus instruments that measure surface roughness. These electroless-nickel coated steel blocks have a sinusoidal roughness profile machined on the top surface.

$\begin{array}{lll}\text { SRM } & \begin{array}{l}\text { Roughness, } \\ \mathbf{R}_{\mathrm{a}}(\boldsymbol{\mu m})\end{array} & \begin{array}{l}\text { Wavelength, } \\ \mathbf{D}(\mu \mathrm{m})\end{array} \\ & & \\ & \\ \text { Sinusoidal Roughness (Knoop Hardness 500) } & \\ 2071 \mathrm{~b} & 0.3137 & 100 \\ 2073 a & 0.034 & 100 \\ 2074 & 0.025 & 40 \\ 2075 & 0.012 & 800\end{array}$

\section{FIRE RESEAR C H}

\section{Surface Flammability}

This SRM is suitable for checking the operation of radiant panel test equipment in accordance with ASTM E 162-78.

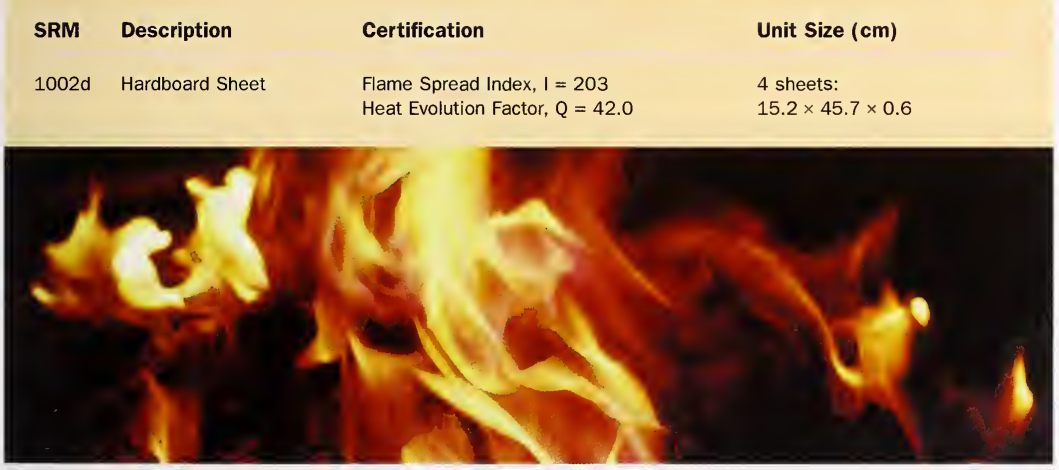




\section{Smoke Density Chamber}

These SRMs are suitable for use with National Fire Protection Agency (NFPA) 258-1998. SRM 1006d is also suitable for use with ASTM E 662-95.

$\begin{array}{cl}\text { SRM } & \text { Description } \\ 1006 \mathrm{~d} & \begin{array}{l}\text { Non-Flaming Exposure } \\ \text { Condition (paper) }\end{array} \\ 1007 \mathrm{~b} & \begin{array}{l}\text { Flaming Exposure } \\ \text { Condition (plastic) }\end{array}\end{array}$

Maximum Specific

Optical Density $\left(D_{m}\right.$ (corr.))

193

388 to 512
Unit Size (cm)

9 sheets:

$17.2 \times 25.4 \times 0.165$

1 sheet:

$25.4 \times 25.4 \times 0.076$

\section{Smoke Toxicity}

\begin{tabular}{|c|c|c|c|c|c|c|}
\hline \multirow[t]{2}{*}{ SRM } & \multirow[t]{2}{*}{ Description } & \multirow{2}{*}{$\begin{array}{l}\text { Combustion } \\
\text { on Mode }\end{array}$} & \multirow{2}{*}{$\begin{array}{l}\text { Observation } \\
\text { Time }\end{array}$} & \multicolumn{2}{|c|}{ Values } & \multirow{2}{*}{$\begin{array}{l}\text { Unit } \\
\text { Size }\end{array}$} \\
\hline & & & & $\mathbf{L C}_{50}$ & $\overline{\text { N-Gas }}$ & \\
\hline 1048 & $\begin{array}{l}\text { Cup Furnace Smoke } \\
\text { Toxicity Method } \\
\text { Standard } \\
\text { (ABS copolymer) }\end{array}$ & NonFlaming & $\begin{array}{l}\text { WE* } \\
\text { WE \& PE** } \\
\text { WE* } \\
\text { WE \& PE** }\end{array}$ & $\begin{array}{l}27 \\
25 \\
58 \\
53\end{array}$ & $\begin{array}{l}1.4 \\
1.5 \\
1.2 \\
1.2 \\
1.4\end{array}$ & $\begin{array}{l}8 \text { sheets: } \\
(16 \times 16 \times 0.76) \mathrm{mm}\end{array}$ \\
\hline 1049 & $\begin{array}{l}\text { University of Pittsburgh } \\
\text { I Smoke Toxicity Method } \\
\text { Standard } \\
\text { (Nylon 6/6) }\end{array}$ & & $\begin{array}{l}30 \text { min exposure, } \\
\text { plus } 10 \mathrm{~min} \\
\text { post-exposure }\end{array}$ & 4.4 & & $150 \mathrm{~g}$ \\
\hline
\end{tabular}

\section{Flooring Radiant Panel}

This SRM is suitable for use with ASTM E 648-78 and NFPA 253-1978.

$\begin{array}{ll}\text { SRM } & \text { Description } \\ 1012 & \begin{array}{l}\text { Flooring Radiant Panel } \\ \text { (Kraft Paperboard) }\end{array}\end{array}$

\section{Critical Radiant Flux}

$0.36 \mathrm{~W} / \mathrm{cm}^{2}$

\section{Unit Size $(\mathrm{cm})$}

3 sheets:

$104.1 \times 25.4 \times 0.305$ 


\section{NONDESTRUCTIVE EVALUATION}

\section{Artificial Flaw for Eddy Current NDE}

$\begin{array}{ll}\text { RM } & \text { Description } \\ 8458 & \text { Artificial Flaw (Aluminum Alloy) }\end{array}$

Flaw Size

Unit Size

$3.0 \mathrm{~mm} \times 0.1 \mathrm{~mm}$

$7 \mathrm{~cm} \times 7 \mathrm{~cm} \times 2 \mathrm{~cm}$

\section{PERFOR M A C E NGINEERING MATERIALS}

\section{Fracture Toughness of Steels (Charpy V-Notch Test Blocks)}

Unit Size: set of $10 \mathrm{~mm} \times 10 \mathrm{~mm} \times 54 \mathrm{~mm}$ specimens

These SRMs are suitable for use with ASTM E 23 and ISO/DIS 12736.

$\begin{array}{ll}\text { SRM } & \text { Description } \\ 2092 & \text { Low Energy (4340 Alloy Steel) } \\ 2096 & \text { High Energy (4340 Alloy Steel) } \\ 2098 & \text { Super High Energy (Maraging Steel) }\end{array}$

Energy Range (J)

13 to 20

88 to 136

176 to 244

\section{Rockwell Hardness}

Unit size: $60 \mathrm{~mm}$ diameter $\times 15 \mathrm{~mm}$

$\begin{array}{lll}\text { SRM } & \text { Description } & \text { Nominal Hardness (HRC) } \\ 2810 & \text { Rockwell C Scale Hardness - Low Range } & 25 \\ 2811 & \text { Rockwell C Scale Hardness - Mid Range } & 45 \\ 2812 & \text { Rockwell C Scale Hardness - High Range } & 62\end{array}$




\section{Microindentation Hardness (Knoop and Vickers Test Blocks)}

Unit Size: $1.15 \mathrm{~cm} \times 1.15 \mathrm{~cm}$ (unless otherwise noted)

These SRMs are suitable for use with ASTM E 384.

$\begin{array}{lll}\text { SRM Description } \quad \text { Load }(\mathrm{N}) & \begin{array}{l}\text { Hardness } \\ \left(\mathrm{kg} / \mathrm{mm}^{2}\right)\end{array}\end{array}$

Copper, Bright

1893

Knoop

$0.245,0.49,0.98$

125

Nickel, Bright

1895

Knoop

$0.245,0.49,0.98$

600

$1896 a$

Vickers

$0.245,0.49,0.98$

600

1905

Knoop

2.943

600

1906

Knoop

4.905

600

1907

Knoop

9.81

600

Silicon Nitride, Ceramic

$2830(22 \mathrm{~mm}$ diameter $\times 9.54 \mathrm{~mm})$

Knoop

19.6

1500

\section{Coordinate Measuring Machine (CMM) Probe Performance}

These SRMs are suitable for use with ANSI/ASME B89.4.1.

$\begin{array}{ll}\text { SRM } & \text { Description } \\ 2084 & \text { Tungsten Carbide Sphere } \\ 2084 \mathrm{R} & \text { Tungsten Carbide Sphere } \\ 2085 & \text { Stainless Steel Sphere }\end{array}$
Unit Size
$10 \mathrm{~mm}$ diameter (stem mounted with stand)
$10 \mathrm{~mm}$ diameter (stem mounted)
$25 \mathrm{~mm}$ diameter (stem mounted)

\section{Tape Adhesion Testing}

This SRM is suitable for use with ASTM D 2860 and ASTM D 3654 .

SRM Description
1810a Linerboard for Tape Adhesion Testing

\section{Unit Size}

50 sheets: $21.6 \mathrm{~cm} \times 28 \mathrm{~cm}$ 


\section{Bleached Kraft Pulps}

These RMs are intended primarily for use in fundamental studies on the physical properties of fibers and paper sheets. No extensive property measurements have been made on these materials beyond ensuring that they were within the control limits of the normal production run.

\begin{tabular}{lll} 
RM & Description & Unit Size \\
\hline $8495^{*}$ & Northern Softwood & 10 standard lap sheets: $0.5 \mathrm{~kg}$ each \\
$8496 *$ & Eucalyptus Hardwood & 10 standard lap sheets: $0.5 \mathrm{~kg}$ each
\end{tabular}

*Developed in cooperation with the Pulp Material Research Committee

\section{Secondary Ferrite Number (FN) Materials}

The RMs are suitable for use with ANSI/AWS A4.2 and IS0 8249.
RM Ferrite Number
Unit Size (mm)
$8480 \quad 0$ to 30
$10 \times 12 \times 20$
848130 to 120
$10 \times 12 \times 20$

\section{Fracture Toughness of Ceramics}

Unit Size: $3 \mathrm{~mm} \times 4 \mathrm{~mm} \times(45$ to 47$) \mathrm{mm}$

$\begin{array}{llll}\text { SRM } & \text { Description } & \begin{array}{l}\text { Fracture Toughness } \\ \left(\mathbf{M P a} \cdot \mathbf{m}^{1 / 2}\right)\end{array} & \begin{array}{l}\text { No. of } \\ \text { Specimens }\end{array} \\ 2100 & \text { Silicon Nitride Flexure Specimens } & 4.57 & 5\end{array}$

\section{Magnetic Moment Standards}

$\begin{array}{llll}\text { SRM } & \text { Description } & \text { Certified Property } & \text { Unit Size } \\ 762 & \text { Nickel Disk } & \text { Specific Magnetization } & \text { disk: } 6 \mathrm{~mm} \text { diameter } \times 0.13 \mathrm{~mm} \\ 772 a & \text { Nickel Sphere } & \text { Magnetic Moment } & \text { sphere: } 2.383 \mathrm{~mm} \text { diameter sphere }\end{array}$




\section{FOOD \& AGRGULURE}

9 Trace Elements in Food and Dairy Products

9 Wheat Hardness

10 Nutrition Composition

11 Trace Elements in Botanicals

11 Fertilizers

11 Whole Blomass Feedstock

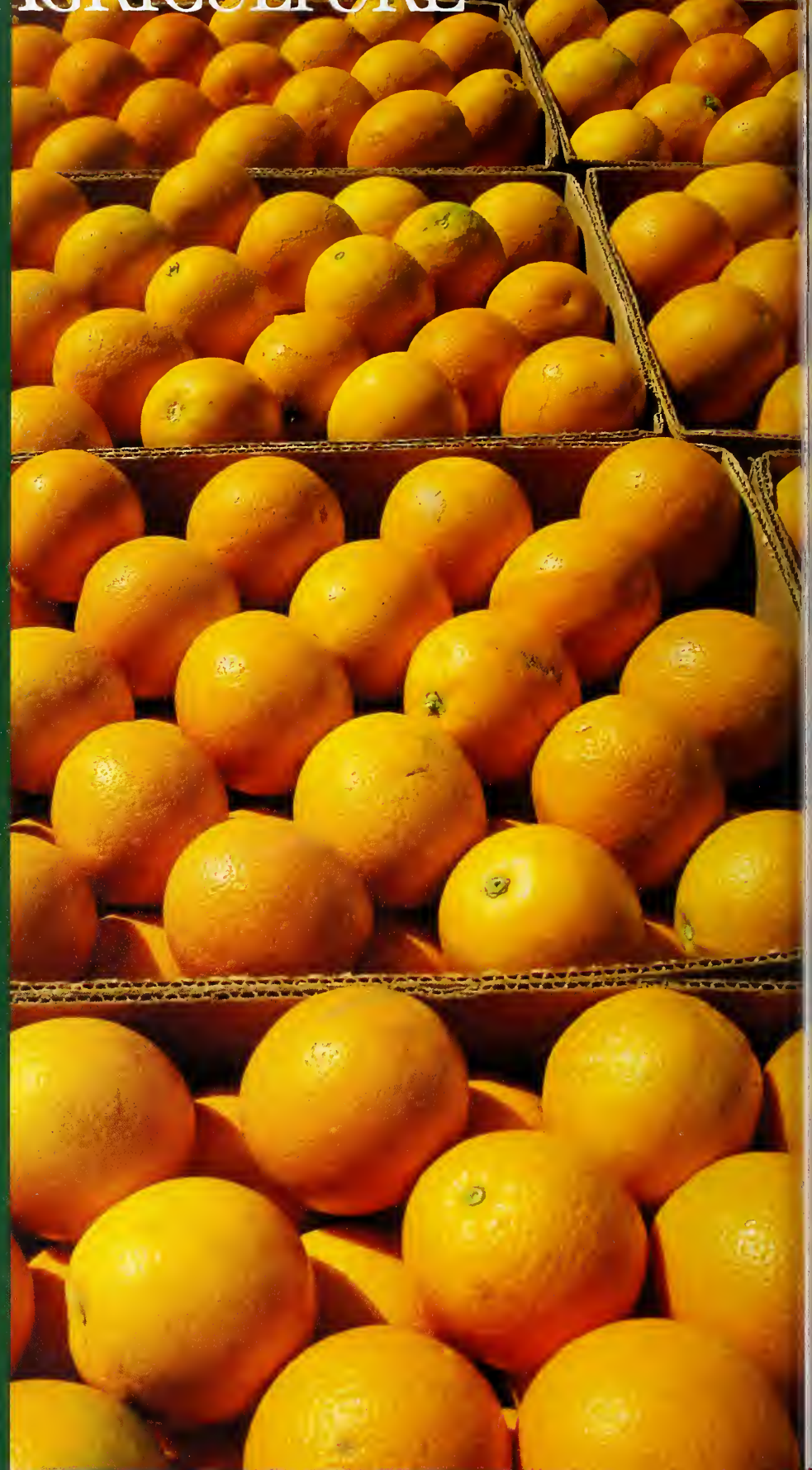


Trace Elements in Food and Dairy Products

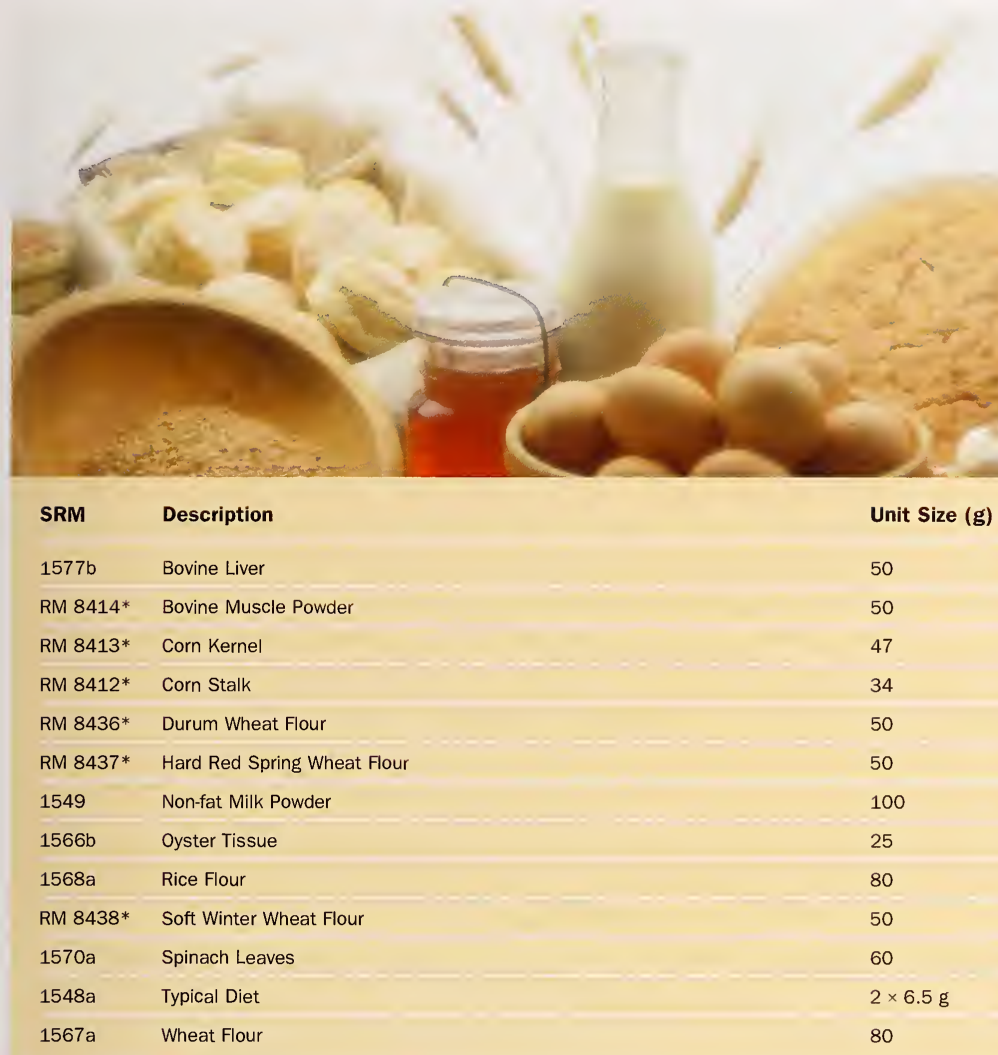

* Developed by Agriculture Canada in cooperation with NIST

\section{Wheat Hardness}

Unit Size: $50 \times 20 \mathrm{~g}$

RM Description

8441* Wheat Hardness

\section{Wheat Numbers}

Hard-1 through Hard-5

Soft-1 through Soft-5 


\section{Nutrition Composition}

Please visit our website to view the relevant certificate or report of investigation for available certified and non-certified values.

\section{NIST Food-Matrix SRMs and RMs}

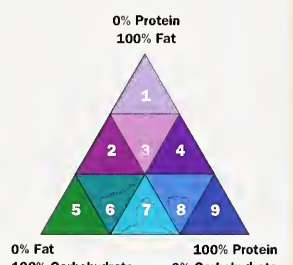

1. SRM 1563

2. SRM 2384

3. SRM $2387^{*}$

4. SRM 1546 RM 8415

5. SRM 2383 RM 8432

RM 8433 RM 8436

6. SRM 1846 RM 8435

SRM 1548 a SRM 1544

7. SRM 1566b SRM 1570a SRM 2385*

9. SRM 1946* SRM 1947* SRM 1974a RM 8418

*In prep

\begin{tabular}{|c|c|}
\hline SRM & Description \\
\hline 1544 & $\begin{array}{l}\text { Fatty Acids and Cholesterol } \\
\text { in Frozen Diet Composite }\end{array}$ \\
\hline 1546 & Meat Homogenate \\
\hline $1548 a$ & Typical Diet \\
\hline 1563 & $\begin{array}{l}\text { Cholesterol and Fat-Soluble } \\
\text { Vitamins in Coconut Oil }\end{array}$ \\
\hline $1589 a$ & $\begin{array}{l}\text { PCBs, Pesticides, and Dioxins/ } \\
\text { Furans in Human Serum }\end{array}$ \\
\hline 1845 & Whole Egg Powder \\
\hline 1846 & Infant Formula (milk-based) \\
\hline 2383 & Baby Food Composite \\
\hline
\end{tabular}

RM 8415* Whole Egg Powder

RM 8418* Wheat Gluten

RM 8432* Corn Starch

RM 8433* Corn Bran

RM 8435* Whole Milk Powder

RM 8436* Durum Wheat Flour

1570a Spinach Leaves

2384 Baking Chocolate

1566b Oyster Tissue

1974a Organics in Mussel Tissue (Mytilus Edulis)

* Developed by Agriculture Canada in cooperation with NIST

** Proximates are provided as reference values.

\section{Certified Constituents* *}

Cholesterol, Fatty Acids, Proximates

Cholesterol, Fatty Acids, Proximates,

Vitamins, Minerals

Proximates, Trace Elements, Total Dietary Fiber

Cholesterol, Ergocalciferol, dl- $\alpha$-Tocopheryl Acetate

Cholesterol, Triglycerides

Cholesterol

Minerals, Proximates, Vitamins, Fatty Acids

Carotenoids, Cholesterol, Minerals,

Proximates, Vitamins

Fatty Acids, Minerals, Proximates, Vitamins

Fatty Acids, Minerals, Proximates, Vitamins

Fatty Acids, Minerals, Proximates, Vitamins

Fatty Acids, Minerals, Proximates, Vitamins

Fatty Acids, Minerals, Proximates, Vitamins

Fatty Acids, Minerals, Proximates, Vitamins

Fatty Acids, Trace Elements, Proximates,

Total Dietary Fibers

Fat, Fatty Acids, Calcium, Iron, Caffeine, Theobromine, Catechins

Fatty Acids, Nitrogen, Proximates, Total Dietary Fiber, Trace Elements, Mercury, Methylmercury

Selected Proximate

$3 \times 15 \mathrm{~g}$

Unit Size (g)

$4 \times 15 g$

$4 \times 85 g$

$2 \times 6.5 \mathrm{~g}$

10 ampoules:

5 fortified,

5 natural

$5 \times 10 \mathrm{~mL}$

35

$10 \times 30 \mathrm{~g}$

$4 \times 70 \mathrm{~g}$

35

50

50

50

40

50

60

$5 \times 91 g$

25

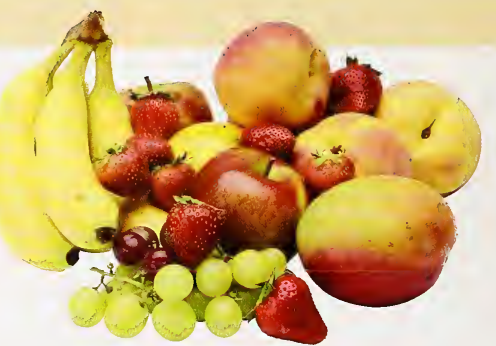




\section{Trace Elements in Botanicals}

$\begin{array}{lll}\text { SRM } & \text { Description } & \text { Unit Size (g) } \\ 1515 & \text { Apple Leaves } & 50 \\ 1547 & \text { Peach Leaves } & 50 \\ 1570 \mathrm{a} & \text { Spinach Leaves } & 50 \\ 1573 a & \text { Tomato Leaves } & 2 \times 25 \mathrm{~g} \\ 2695 * & \text { Fluoride in Vegetation } & 34 \\ \text { RM 8412 } & \text { Corn Stalk (Zea Mays) } & 47 \\ \text { RM } 8413 & \text { Corn Kernel (Zea Mays) } & \end{array}$

* Developed in cooperation with Aluminum Association, Inc.

\section{Fertilizers}

Unit Size: $90 \mathrm{~g}$

$\begin{array}{lll}\text { SRM } & \text { Description } & \text { Certified } \\ 120 \mathrm{c} & \text { Phosphate Rock (Florida) } & \text { Minerals } \\ 193 & \text { Potassium Nitrate } & \mathrm{N}, \mathrm{K} \\ 194 & \text { Ammonium Dihydrogen Phosphate } & \mathrm{N}, \mathrm{P} \\ 200 \mathrm{a} & \text { Potassium Dihydrogen Phosphate } & \mathrm{K}, \mathrm{P} \\ 694 & \text { Phosphate Rock (Western) } & \text { Minerals }\end{array}$

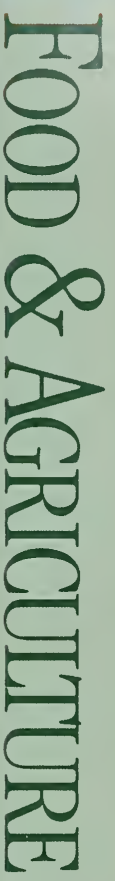

\section{Whole Biomass Feedstock*}

Unit Size: $5 \times 10 \mathrm{~g}$

$\begin{array}{lll}\text { RM } & \text { Description } & \text { Reference Constituents } \\ 8491 & \text { Sugarcane Bagasse } & \text { Ash, Ethanol Extractives, Acid Soluble Lignin, } \\ 8492 & \text { Populus Deltoides } & \text { Acid Insoluble Lignin, Total Lignin, Glucuronic Acid, } \\ 8493 & \text { Monterey Pine } & \text { Arabinan, Xylan, Mannan, Galactan, Glucan } \\ 8494 & \text { Wheat Straw } & \end{array}$

* Developed by the International Energy Agency (IEA) Biomass Annex, NREL, and NIST 


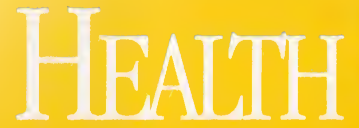

13 Pure, Crystalline Standards

13 Biological Buffer Systems

14 Human Serum

15 Bovine Serum

15 Ethanol Solutions

16 DNA Profiling

16 Biomaterials

17 Drugs of Abuse in Urine

17 Toxic Substances in Urine

17 Miscellaneous HealthRelated Standards 
Pure, Crystalline Standards

\begin{tabular}{|c|c|c|c|}
\hline SRM & Description & Purity (\%) & Unit Size (g) \\
\hline 938 & 4-Nitrophenol & $(99.75)^{*}$ & 15 \\
\hline 998 & Angiotensin I (Human) & 94.1 & 0.5 \\
\hline $916 a$ & Bilirubin & 98.3 & 0.1 \\
\hline $915 a$ & Calcium Carbonate & 99.9 & 20 \\
\hline $911 b$ & Cholesterol & 99.8 & 2 \\
\hline 921 & Cortisol (Hydrocortisone) & 98.9 & 1 \\
\hline $914 a$ & Creatinine & 99.7 & 10 \\
\hline $917 \mathrm{~b}$ & D-Glucose (Dextrose) & 99.7 & 50 \\
\hline 920 & D-Mannitol & 99.8 & 50 \\
\hline 937 & Iron Metal (Clinical) & 99.90 & 50 \\
\hline 928 & Lead Nitrate & 100.00 & 30 \\
\hline $924 a$ & Lithium Carbonate & 99.867 & 30 \\
\hline 929 & Magnesium Gluconate Dihydrate & $5.403 \mathrm{Mg}$ & 5 \\
\hline $918 a$ & Potassium Chloride & 99.9817 & 30 \\
\hline $919 a$ & Sodium Chloride & 99.89 & 30 \\
\hline 910 & Sodium Pyruvate & 98.7 & 25 \\
\hline 1595 & Tripalmitin & 99.5 & 2 \\
\hline $912 \mathrm{a}$ & Urea & 99.9 & 25 \\
\hline $913 a$ & Uric Acid & 99.6 & 10 \\
\hline 925 & VMA (4-hydroxy-3-methoxy-DL-mandelic acid) & 99.4 & 1 \\
\hline
\end{tabular}

*Values in parentheses are not certified and are given for information only.

\section{Biological Buffer Systems}

Unit Size: 60 grams

\begin{tabular}{|c|c|c|c|}
\hline \multirow[t]{2}{*}{ SRM } & \multirow[t]{2}{*}{ Description } & \multicolumn{2}{|c|}{$\mathrm{pH}(\mathrm{S})$ Values (at $37^{\circ} \mathrm{C}$ ) } \\
\hline & & 0.05 molal & 0.08 molal \\
\hline $\begin{array}{l}2181 \\
2182\end{array}$ & $\begin{array}{l}\text { HEPES Free Acid } \\
\text { NaHEPESate }\end{array}$ & $7.364 *$ & $7.373^{*}$ \\
\hline $\begin{array}{l}2183 \\
2184\end{array}$ & $\begin{array}{l}\text { MOPSO Free Acid } \\
\text { NaMOPSOate }\end{array}$ & $6.699 *$ & $6.694 *$ \\
\hline
\end{tabular}

*This $\mathrm{pH}$ results only when the two SRMs listed are used as an admixture in solution. 


\section{Human Serum}

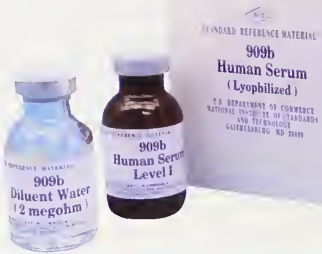

$\begin{array}{cl}\text { SRM } & \text { Description } \\ 1599 & \begin{array}{l}\text { Anticonvulsant } \\ \text { Drug Level Assay } \\ \text { (Valproic Acid and } \\ \text { Carbamazepine) }\end{array}\end{array}$

\section{Certified Constituents}

Reference Values

Form

No. of

Constituents

Levels

PCB Congeners (16),

PCB Congeners (9),

Lyophilized

Chlorinated Pesticides (5),

Total Cholesterol

Chlorinated Pesticides (5),

Total Cholesterol,

Triglycerides, "Free"

Cholesterol, Phospholipids

\begin{tabular}{|c|c|c|c|c|}
\hline 900 & $\begin{array}{l}\text { Antiepilepsy Drug } \\
\text { Level Assay }\end{array}$ & Antiepileptics (4) & - & Lyophilized \\
\hline 970 & $\begin{array}{l}\text { Ascorbic Acid in } \\
\text { Frozen Human } \\
\text { Serum }\end{array}$ & Total Ascorbic Acid & - & Frozen \\
\hline $1952 a$ & $\begin{array}{l}\text { Cholesterol in } \\
\text { Human Serum }\end{array}$ & Cholesterol & - & Lyophilized \\
\hline $956 a$ & $\begin{array}{l}\text { Electrolytes } \\
\text { in Frozen } \\
\text { Human Serum }\end{array}$ & Total Ca, Li, Mg, K, Na & Ionized $\mathrm{Ca}, \mathrm{Cl}$ & Frozen \\
\hline $968 \mathrm{c}$ & $\begin{array}{l}\text { Fat-Soluble } \\
\text { Vitamins, } \\
\text { Carotenoids, } \\
\text { and Cholesterol } \\
\text { in Human Serum }\end{array}$ & $\begin{array}{l}\text { Vitamins (4), } \\
\text { Cholesterol, } \\
\text { Carotenoids (4) }\end{array}$ & $\begin{array}{l}\text { Carotenoids (8), } \\
\text { Vitamin D }\end{array}$ & Lyophilized \\
\hline 965 & $\begin{array}{l}\text { Glucose in } \\
\text { Human Serum }\end{array}$ & Glucose & - & Frozen \\
\hline $909 b$ & Human Serum & $\begin{array}{l}\text { Organics (6), } \\
\text { Inorganics (6) }\end{array}$ & Bilirubin & Lyophilized \\
\hline $1951 a$ & Lipids in Frozen & $\begin{array}{l}\text { Total Cholesterol, } \\
\text { Total Glycerides } \\
\text { Triglycerides }\end{array}$ & $\begin{array}{l}\text { HDL-, LDL-, and } \\
\text { Total Cholesterol, } \\
\text { Triglycerides, } \\
\text { Free Glycerol }\end{array}$ & \\
\hline $1589 a$ & $\begin{array}{l}\text { PCBs, Pesticides, } \\
\text { Dioxins/Furans in } \\
\text { Serum }\end{array}$ & $\begin{array}{l}\text { PCB Congeners (16), } \\
\text { Chlorinated Pesticides (5), } \\
\text { Total Cholesterol }\end{array}$ & $\begin{array}{l}\text { PCB Congeners (9), } \\
\text { Chlorinated Pesticides (5), } \\
\text { Total Cholesterol (5), } \\
\text { Triglycerides, "Free" } \\
\text { Cholesterol, Phospholipids }\end{array}$ & Lyophilized \\
\hline
\end{tabular}




\section{Bovine Serum}

$\begin{array}{llllll}\text { SRM } & \text { Description } & \begin{array}{l}\text { Certified } \\ \text { Constituents }\end{array} & \begin{array}{l}\text { Reference } \\ \text { Constituents }\end{array} & \text { Form } & \begin{array}{l}\text { No. of } \\ \text { Levels }\end{array} \\ 927 c & \begin{array}{l}\text { Bovine Serum } \\ \text { Albumin (7\% Solution) }\end{array} & \text { Protein Concentration } & - & \text { Solution } & 1 \\ 1598 & \begin{array}{l}\text { Inorganic Constituents } \\ \text { in Bovine Serum }\end{array} & \text { Elements (13) } & - & \text { Frozen } & 1 \\ 955 b & \text { Lead in Bovine Blood } & \mathrm{Pb} & - & \text { Frozen } & 4 \\ 966 & \begin{array}{l}\text { Toxic Elements in } \\ \text { Bovine Blood }\end{array} & \mathrm{Pb}, \mathrm{Cd} & \text { Pb, Cd, Total Hg, } & \text { Frozen } & 2\end{array}$

\section{Ethanol Solutions}

This SRM is for use in the calibration of instruments and techniques for the determination of ethanol (ethyl alcohol) in breath and blood.
SRM
Description
Ethanol Mass
Fraction (\%)
Unit Size
$1828 \mathrm{a}$
Ethanol - Water Solutions
(Set: 5 ampoules)
95.60
$1 \times 20 \mathrm{~mL}$

1.9957
0.09480
0.02186
$2 \times 20 \mathrm{~mL}$
$1 \times 5 \mathrm{~mL}$
$1 \times 5 \mathrm{~mL}$

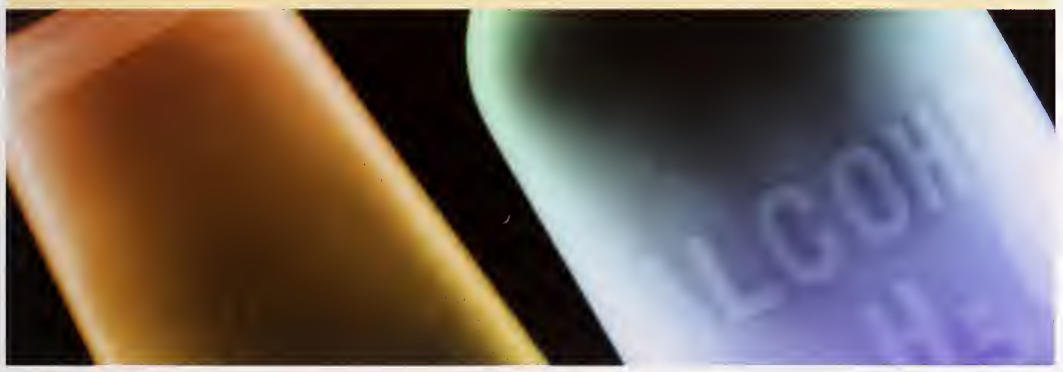




\section{DNA Profiling}

SRMs 2390 , and 2391a are intended for use in the standardization of forensic and paternity quality assurance procedures and instructional law enforcement or non-clinical research purposes. SRM 2392 is intended to provide quality control when performing the polymerase chain reaction (PCR) and sequencing of human mitochondrial DNA (mtDNA) for forensic identifications, medical diagnosis, or mutation detection. It may also be used as a control when amplifying (PCR) and sequencing any DNA.

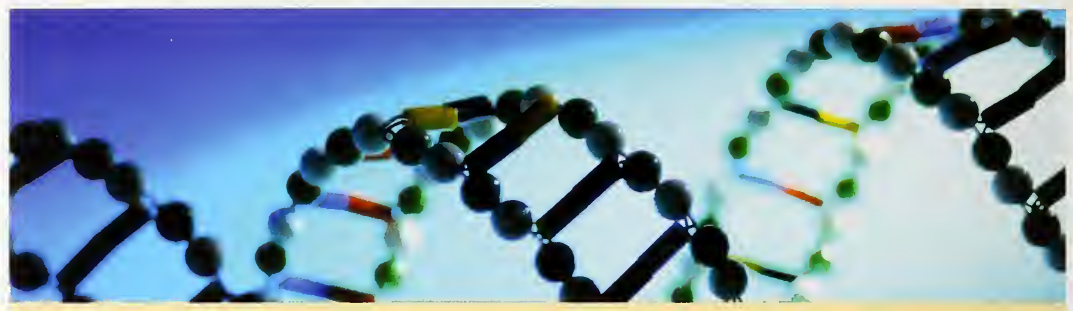

SRM Description

2390 DNA Profiling Standard - RFLP

2391a PCR-Based DNA Profiling Standard

2392 Mitochrondrial DNA Sequencing (Human)

\section{Unit Size}

20 components

12 components

3 components

\section{Biomaterials}

$\begin{array}{ll}\text { SRM } & \text { Description } \\ 2910 & \text { Calcium } \\ & \text { Hydroxyapatite } \\ & \\ \text { RM 8456 } & \begin{array}{l}\text { Ultra High Molecular } \\ \text { Weight Polyethylene }\end{array}\end{array}$

\section{Certified Properties}

Calcium Phosphorus

$\mathrm{Ca} / \mathrm{P}$ Molar Ratio Specific Surface Area Solubility Product

\section{Reference Properties}

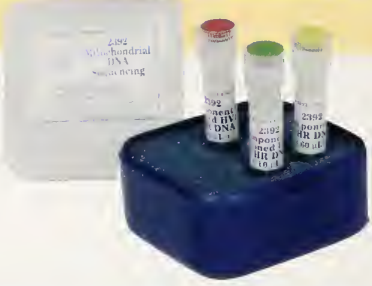

Young's Modulus

Yield Strength

Ultimate Strength

Elongation

\section{Unit Size}

$5 \mathrm{~g}$ (powder)

3 in diameter $\times 60$ in (bar) $(7.62 \mathrm{~cm}$ diameter $\times 152.4 \mathrm{~cm}$ ) 


\section{Drugs of Abuse in Urine}

\begin{tabular}{|c|c|c|c|c|c|}
\hline SRM & Description & $\begin{array}{l}\text { Certified } \\
\text { Constituents }\end{array}$ & $\begin{array}{l}\text { Reference } \\
\text { Constituent }\end{array}$ & Form & Unit Size \\
\hline 1508 & $\begin{array}{l}\text { Cocaine Metabolites } \\
\text { in Urine }\end{array}$ & Benzoylecgonine & & Lyophilized & $\begin{array}{l}3 \text { levels, } \\
\text { plus } 1 \text { blank }\end{array}$ \\
\hline RM 8444 & Cotinine in Urine & & $\begin{array}{l}\text { Cotinine (nicotine } \\
\text { metabolite) }\end{array}$ & Lyophilized & $\begin{array}{l}2 \text { levels, } \\
\text { plus } 1 \text { blank }\end{array}$ \\
\hline $1507 \mathrm{~b}$ & $\begin{array}{l}\text { Marijuana Metabolites } \\
\text { in Urine }\end{array}$ & $\mathrm{TH}-9-\mathrm{COOH}$ & & Lyophilized & $\begin{array}{l}3 \text { levels, } \\
\text { plus } 1 \text { blank }\end{array}$ \\
\hline 2381 & $\begin{array}{l}\text { Morphine and Codeine } \\
\text { in Urine }\end{array}$ & $\begin{array}{l}\text { Morphine and } \\
\text { Codeine }\end{array}$ & & Lyophilized & $\begin{array}{l}3 \text { levels, } \\
\text { plus } 1 \text { blank }\end{array}$ \\
\hline 2382 & $\begin{array}{l}\text { Morphine Glucuronide } \\
\text { in Urine }\end{array}$ & Free Morphine & & Lyophilized & $\begin{array}{l}3 \text { levels, } \\
\text { plus } 1 \text { blank }\end{array}$ \\
\hline 1511 & $\begin{array}{l}\text { Multi Drugs of Abuse } \\
\text { in Urine }\end{array}$ & Drugs of Abuse (5) & & Lyophilized & 1 level \\
\hline
\end{tabular}

\section{Toxic Substances in Urine}

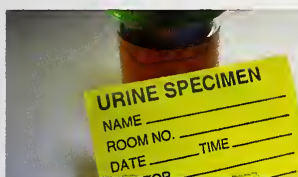

$\begin{array}{ll}\text { SRM } & \text { Description } \\ 2671 a & \text { Fluoride } \\ 2672 a & \text { Mercury }\end{array}$

No. of Levels

Unit Size

$4 \times 20 \mathrm{~mL}$

$4 \times 20 \mathrm{~mL}$

\section{Miscellaneous Health - Related Materials}

$\begin{array}{lllll}\text { SRM } & \text { Description } & \text { Certified Constituents } & \text { Form } & \text { Unit Size } \\ 2389 & \begin{array}{l}\text { Amino Acids in } \\ 0.1 \mathrm{~mol} / \mathrm{L} \mathrm{HCl}\end{array} & \text { Amino Acids (17) } & \text { Solution } & 5 \text { ampoules } \\ 1400 & \text { Bone Ash } & \text { Elements (8) } & \text { Powdered } & 50 \mathrm{~g} \\ 1486 & \text { Bone Meal } & \text { Elements (8) } & \text { Powdered } & 50 \mathrm{~g}\end{array}$


ENVIRONMIS SE 10

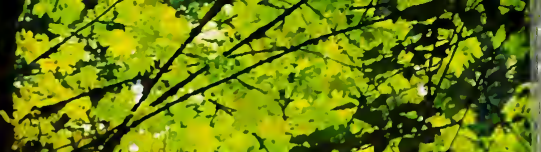

19 Organles

23 Inorganics

28 Fossil Fuels

32 Geological Materials and Ores 5.

35 Mlcroanalysis

36 Engine Wear Materials

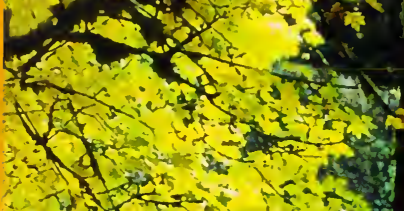

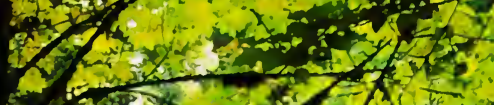

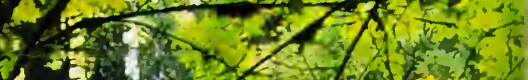

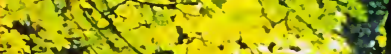

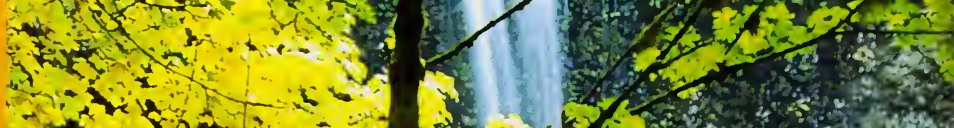

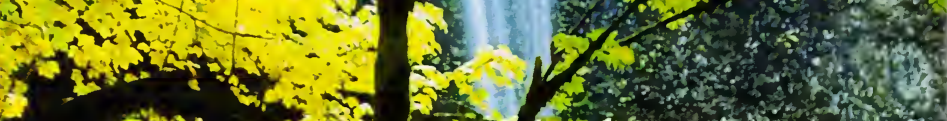

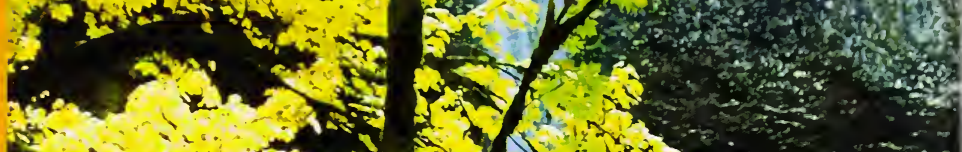

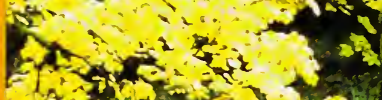

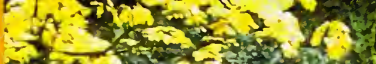

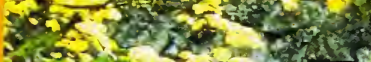

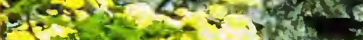

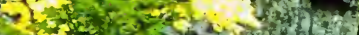

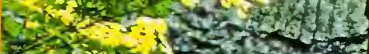

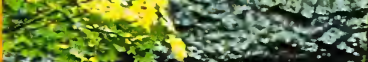

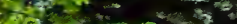

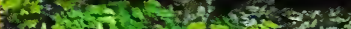
7 t.

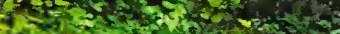

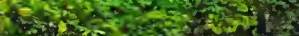

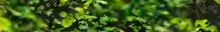
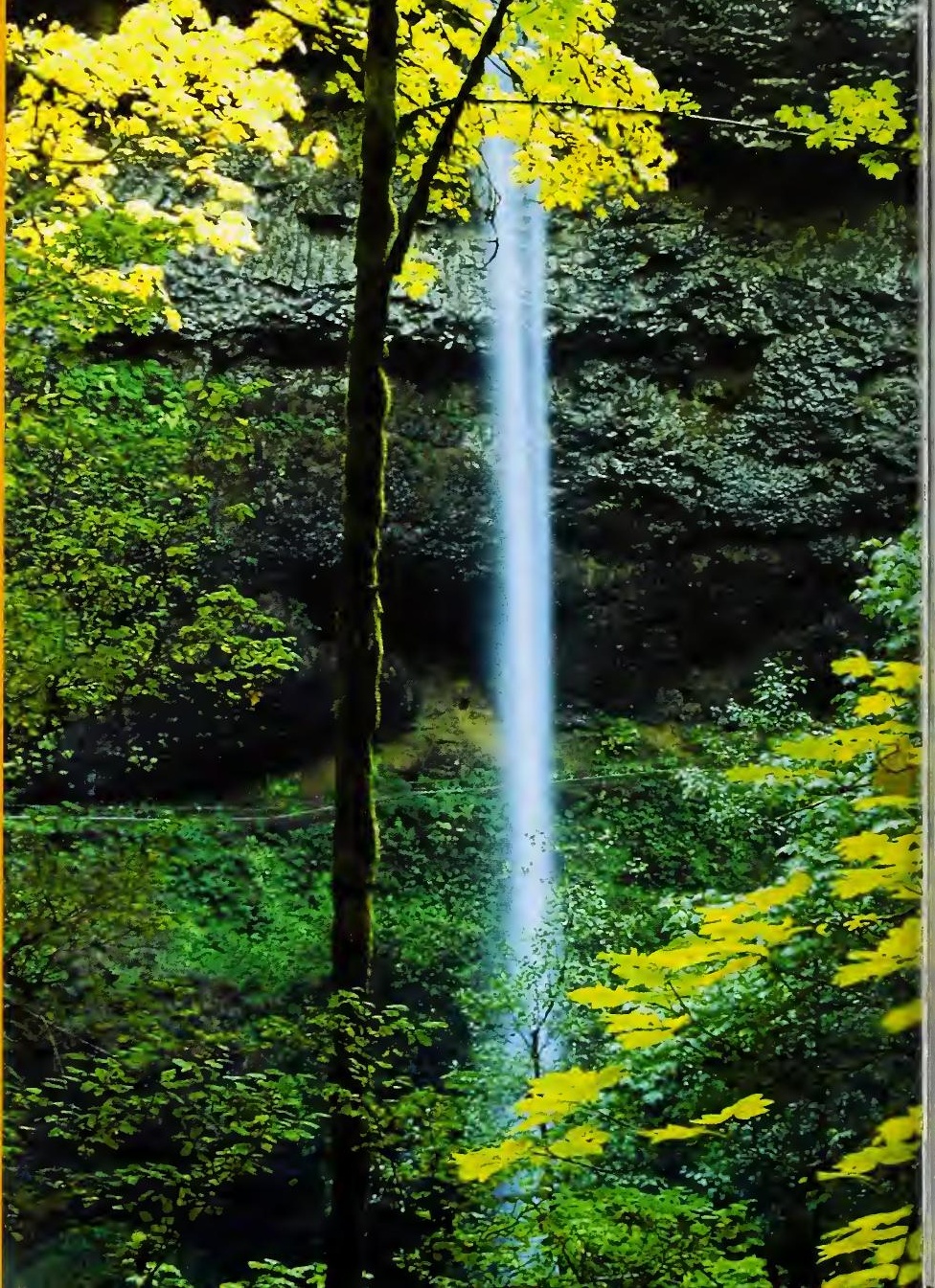

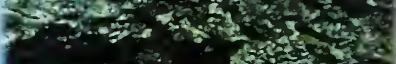




\section{OR G A N I C S}

Gas Chromatography/Mass Spectrometry (GC/MS) and Characterizing Liquid Chromatography (LC ) System Performance

\begin{tabular}{|c|c|c|c|c|c|}
\hline SRM & Description & $\begin{array}{l}\text { Certified } \\
\text { Constituents }\end{array}$ & Solvent & $\begin{array}{l}\text { No. of } \\
\text { Levels }\end{array}$ & Unit Size \\
\hline 1543 & GC/MS System & Methyl Stearate, Benzophenone & Hexane & 2 & $4 \times 1 \mathrm{~mL}$ \\
\hline RM 8443 & $\begin{array}{l}\text { Consists of } 5 \text { units } \\
\text { of SRM } 1543\end{array}$ & & & & \\
\hline 877 & LC Chiral Selectivity & various Chiral components & Ethanol & - & $5 \times 1 \mathrm{~mL}$ \\
\hline 870 & LC Performance & $\begin{array}{l}\text { Silanol Activity,Trace Metal } \\
\text { Activity, Hydrophobic Retention, } \\
\text { Methylene Selectivity }\end{array}$ & Methanol & 1 & $5 \times 1 \mathrm{~mL}$ \\
\hline $869 a$ & LC Selectivity & Shape Selectivity: PAHs (3) & Acetonitrile & 1 & $5 \times 1 \mathrm{~mL}$ \\
\hline
\end{tabular}




\section{Organic Contaminant Calibration Solutions}

\begin{tabular}{|c|c|c|c|c|}
\hline SRM & Description & $\begin{array}{l}\text { Certified } \\
\text { Constituents }\end{array}$ & $\begin{array}{l}\text { Non-Certified } \\
\text { Constituents }\end{array}$ & Unit Size \\
\hline RM 8467 & $4,4^{\prime}$-DDE (neat) & - & - & Vial: $100 \mathrm{mg}$ \\
\hline RM 8469 & 4,4'-DDT (neat) & - & - & Vial: $100 \mathrm{mg}$ \\
\hline RM 8466 & g-HCH (Lindane) (neat) & - & - & Vial: $100 \mathrm{mg}$ \\
\hline 1491 & $\begin{array}{l}\text { Aromatic Hydrocarbons } \\
\text { in Hexane/Toluene }\end{array}$ & PAHs (23) & PAHs (1) & 5 ampoules \\
\hline 2260 & $\begin{array}{l}\text { Aromatic Hydrocarbons } \\
\text { in Toluene }\end{array}$ & PAHs (23) & PAHs (1) & 5 ampoules \\
\hline 1493 & $\begin{array}{l}\text { Chlorinated Biphenyl } \\
\text { Congeners in } 2,2,4 \\
\text {-Trimethylpentane }\end{array}$ & PCBs (18) & PCBs (2) & 5 ampoules \\
\hline 2262 & $\begin{array}{l}\text { Chlorinated Biphenyl } \\
\text { Congeners in } 2,2,4- \\
\text { Trimethylpentane }\end{array}$ & PCBs (25) & PCBs (4) & 5 ampoules \\
\hline 2275 & Chlorinated Pesticide Solution-II & Pesticides (9) & - & 5 ampoules \\
\hline 1492 & Chlorinated Pesticides in Hexane & Pesticides (15) & - & 5 ampoules \\
\hline 2261 & Chlorinated Pesticides in Hexane & Pesticides (15) & - & 5 ampoules \\
\hline 2273 & DDTs and Metabolites in Solution & $\begin{array}{l}\text { DDTs, } \\
\text { Metabolites (7) }\end{array}$ & 5 ampoules & \\
\hline 1596 & $\begin{array}{l}\text { Dinitropyrene Isomers and } \\
\text { 1-Nitropyrene in Methylene Chloride }\end{array}$ & Nitro-PAHs (4) & - & 5 ampoules \\
\hline 1614 & Dioxin $(2,3,7,8$-TCDD) in Iso-octane & Dioxins (2) & Dioxins (2) & 6 ampoules \\
\hline 1639 & $\begin{array}{l}\text { Halocarbons (in Methanol) } \\
\text { for Water Analysis }\end{array}$ & Halocarbons (7) & - & 5 ampoules \\
\hline 1586 & $\begin{array}{l}\text { Isotopically Labeled and Unlabeled } \\
\text { Priority Pollutants in Methanol }\end{array}$ & $\begin{array}{l}\text { Priority pollutants } \\
\text { (10) }\end{array}$ & - & 6 ampoules \\
\hline 1587 & Nitrated PAHs in Methanol & Nitro-PAHs (6) & Nitro-PAHs (1) & 4 ampoules \\
\hline 2274 & PCB Congener Solution-II & PCBs (11) & & 5 ampoules \\
\hline 2269 & Perdeuterated PAH-I & $\begin{array}{l}\text { Perdeuterated } \\
\text { PAHs (5) }\end{array}$ & - & 5 ampoules \\
\hline 2270 & Perdeuterated PAH-II & $\begin{array}{l}\text { Perdeuterated } \\
\text { PAHs (6) }\end{array}$ & - & 5 ampoules \\
\hline $1647 d$ & $\begin{array}{l}\text { Priority Pollutant PAHs } \\
\text { (in Acetonitrile) }\end{array}$ & PAHs (16) & - & 5 ampoules \\
\hline 1584 & $\begin{array}{l}\text { Priority Pollutant Phenols } \\
\text { in Methanol }\end{array}$ & Phenols (10) & Phenols (1) & 5 ampoules \\
\hline 2276 & Three Planar PCBs in Solution & PCBs (3) & - & 5 ampoules \\
\hline
\end{tabular}




\section{Organic Contaminants in Natural Matrix Materials}

\begin{tabular}{|c|c|c|c|c|}
\hline SRM & Description & $\begin{array}{l}\text { Certified } \\
\text { Constituents }\end{array}$ & $\begin{array}{l}\text { Non-Certified } \\
\text { Constituents }\end{array}$ & Unit Size \\
\hline 1597 & $\begin{array}{l}\text { Complex Mixture of } \\
\text { PAHs from Coal Tar }\end{array}$ & PAHs (12) & PAHs/PASH/PANH (18) & 4 ampoules \\
\hline 1975 & Diesel Particulate Extract & PAHs (8) & PAHs (29), Nitro-PAHs & 4 ampoules \\
\hline 1650 & Diesel Particulate Material & PAHs (5), Nitro-PAHs (1) & $\begin{array}{l}\text { PAHs (6), Nitro-PAHs (3), } \\
\text { PAQ (1) }\end{array}$ & $0.01 \mathrm{~g}$ \\
\hline 2975 & Diesel Particulate Matter & $\begin{array}{l}\text { PAHs (11) } \\
\text { (Industrial Forklift) }\end{array}$ & $\begin{array}{l}\text { PAHs (28), Total Extractable } \\
\text { Mass, Particle Size Distribution }\end{array}$ & $1 \mathrm{~g}$ \\
\hline 2978 & $\begin{array}{l}\text { Mussel Tissue } \\
\text { (Organic Contaminants - } \\
\text { Raritan Bay, NJ) }\end{array}$ & $\begin{array}{l}\text { PAHs (7), PCB } \\
\text { Congeners (22), } \\
\text { Pesticides (12) }\end{array}$ & PAHs (20), PCBs (2) & $10 \mathrm{~g}$ \\
\hline 2977 & Mussel Tissue & $\begin{array}{l}\text { PAHs (14), PCB } \\
\text { Congeners (25), } \\
\text { Pesticides (7), Trace } \\
\text { Elements (6), } \\
\text { Methylmercury }\end{array}$ & $\begin{array}{l}\text { PAHs (16), } \\
\text { Trace Elements (9) }\end{array}$ & $10 \mathrm{~g}$ \\
\hline 2976 & Mussel Tissue & $\begin{array}{l}\text { Methylmercury, } \\
\text { Total Mercury, Trace } \\
\text { Elements (7) }\end{array}$ & Trace elements (20) & $25 g$ \\
\hline 1944 & $\begin{array}{l}\text { New York/New Jersey } \\
\text { Waterway Sediment }\end{array}$ & $\begin{array}{l}\text { PAHs (24), PCBs (35), } \\
\text { Pesticides (4), Trace } \\
\text { Elements (9) }\end{array}$ & $\begin{array}{l}\text { PAHs (32), Pesticides ( } 7 \text { ), } \\
\text { Trace Elements (20), } \\
\text { PCDDs/PCDFs (17), Particle } \\
\text { Size, Total Organic Carbon }\end{array}$ & $50 \mathrm{~g}$ \\
\hline $1588 a$ & Organics in Cod Liver Oil & PCBs (24), Pesticides (4) & $\begin{array}{l}\text { PCDDs/PCDFs (7), PCBs (34), } \\
\text { Pesticides (3) }\end{array}$ & 5 ampoules \\
\hline $1974 a$ & $\begin{array}{l}\text { Organics in Mussel Tissue } \\
\text { (Mytilus Edulis) (Frozen) }\end{array}$ & $\begin{array}{l}\text { PAHs (15), PCBs (20), } \\
\text { Pesticides (7), Total } \\
\text { Mercury, Methylmercury }\end{array}$ & $\begin{array}{l}\text { Aliphatics (16), Trace } \\
\text { Elements (32), PAHs (18), } \\
\text { PCBs (4), Pesticides (4), } \\
\text { Proximates, Calories }\end{array}$ & $3 \times 15 g$ \\
\hline 1580 & Organics in Shale Oil & $\begin{array}{l}\text { PAHs (5), Phenols ( } 3 \text { ), } \\
\text { PANH (1) }\end{array}$ & Phenols (6), PANH (1) & 5 ampoules \\
\hline 1945 & $\begin{array}{l}\text { Organics in Whale Blubber } \\
\text { (Frozen) }\end{array}$ & PCBs (27), Pesticides (15) & PCBs (2), Pesticides (2) & 2 bottles \\
\hline $1589 a$ & $\begin{array}{l}\text { PCBs, Pesticides, and } \\
\text { Dioxins/Furans in } \\
\text { Human Serum }\end{array}$ & $\begin{array}{l}\text { PCBs Congeners (16), } \\
\text { Pesticides (5), Total } \\
\text { Cholesterol }\end{array}$ & $\begin{array}{l}\text { CDC Lipid Laboratory: PCB } \\
\text { Cogeners (9), Pesticides } \\
\text { (5), Total Cholesterol, } \\
\text { Triglycerides, "Free" Cholesterol, } \\
\text { Phospholipids, PCDDs, PCDFs, } \\
\text { non-ortho, PCBs }\end{array}$ & $5 \times 10 \mathrm{~mL}$ \\
\hline 1582 & Petroleum Crude Oil & PAHs (5), PASH (1) & PAHs (5), Phenols (2), PANH (1) & 5 ampoules \\
\hline $1939 a$ & $\begin{array}{l}\text { Polychlorinated Biphenyls } \\
\text { (Congeners) in River } \\
\text { Sediment }\end{array}$ & PCBs (20), Pesticides (3) & PCBs (4) & $50 \mathrm{~g}$ \\
\hline $1649 a$ & Urban Dust & $\begin{array}{l}\text { PAHs (22), PCBs (35), } \\
\text { Pesticides (8) }\end{array}$ & $\begin{array}{l}\text { PAHs (22), Pesticide (1, } \\
\text { Mutagenic Activity), PCDD/ } \\
\text { PCDFs (17), Trace } \\
\text { Elements (32), Particle size, } \\
\text { Total Organic Carbon }\end{array}$ & $2.5 \mathrm{~g}$ \\
\hline 1648 & Urban Particulate Matter & Trace Elements (9) & Trace Elements (25), PAH (13) & $2 g$ \\
\hline
\end{tabular}




\section{EPA: Organic Compounds Related to Water Analysis}

Unit Size: $2 \times 2.5 \mathrm{~mL}$

These SRMs are intended primarily for the calibration of instrumentation and validation of methods for volatile or semivolatile organic compound determinations. Because of its miscibility with water, each SRM can also be used to fortify aqueous samples with known amounts of the organic compound.

Laboratory Accreditation: National Voluntary Laboratory Accreditation Program (NVLAP)

SRM
3011
3014
3012
3009
3000
3006
3002
3015
3008
3004
3003
3005
3016
3010
3001
3007

\section{Description}

1,1,1-Trichloroethane in Methanol

1,2,3-Trichloropropane in Methanol

1,2-Dichloroethane in Methanol

1,2-Dichloropropane in Methanol

Benzene in Methanol

Carbon Tetrachloride in Methanol

Ethylbenzene in Methanol

Isopropylbenzene in Methanol

Methylene Chloride in Methanol

$\mathrm{m}$-Xylene in Methanol

o-Xylene in Methanol

p-Xylene in Methanol

sec-Butylbenzene in Methanol

Tetrachloroethene (Tetrachloroethylene) in Methanol

Toluene in Methanol

Vinyldene in Methanol
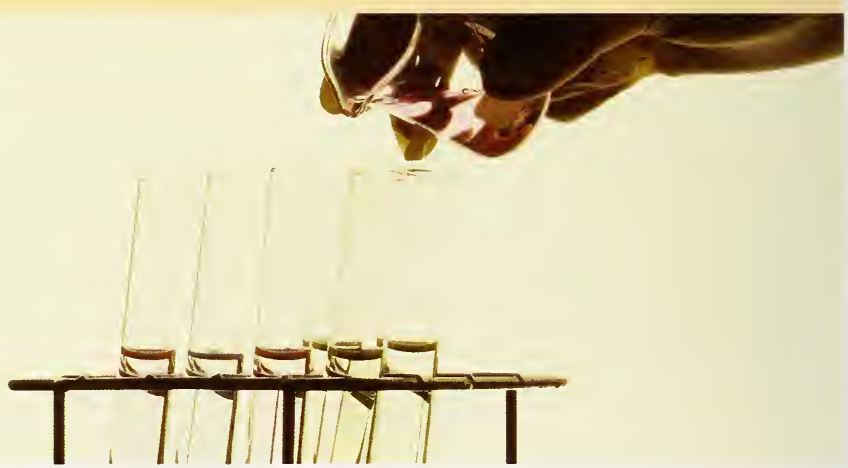

*Developed in support of the Chemical Calibration Providers of the Proficiency Testing Program with support by the U.S. Environmental Protection Agency (EPA). 


\section{NORG A N I C S}

Metal Constituents in Natural Matrices: Air Particulate, Indoor Dust, Sediment, Sludge, Soil, and Water

SRM Description

\section{Air Particulate}

2783

Air Particulate on Filter Media

1648

Urban Particulate Matter

Indoor Dust, Trace Elements in

$2583 \quad$ Nominal $90 \mathrm{mg} / \mathrm{kg}$ Lead

$2584 \quad$ Nominal $1 \%$ Lead

\section{Sediment}

RM 8704 Buffalo River Sediment 25 reference

1646a Estuarine Sediment

1944 New York/New Jersey Waterway Sediment

\section{Sludge}

2781 Domestic Sludge

2782 Industrial Sludge

Soil, Trace Elements in

2710 Montana Soil Highly Elevated Trace Element Concentrations

2711 Montana Soil Moderately Elevated Trace Element Concentrations

2709 San Joaquin Soil

2586 Nominal $500 \mathrm{mg} / \mathrm{kg}$ Lead

$2587 \quad$ Nominal $3000 \mathrm{mg} / \mathrm{kg}$ Lead

Water

1641d Mercury in Water

$1640 \quad$ Natural Water

$1643 e$
Trace Elements in Water (In Prep)
Elements

Unit Size

18 certified

9 reference

15 certified

5 certified

5 certified

10 reference

$8 g$

$50 \mathrm{~g}$

20 certified

72 certified

78 reference

$70 \mathrm{~g}$

$50 \mathrm{~g}$

10 certified

$40 \mathrm{~g}$

10 certified

16 reference

$70 \mathrm{~g}$

21 certified

$50 \mathrm{~g}$

24 certified

$50 \mathrm{~g}$

26 certified

$50 \mathrm{~g}$

4 certified

18 reference

$8 \mathrm{~g}$

4 certified

14 reference

$8 \mathrm{~g}$

2 filters,

plus 2 blanks

$$
\text { g }
$$

$g$

g

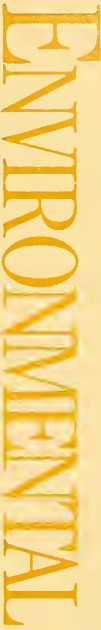




\section{Carbon Modified Silica}

Unit Size: $3 \times 1 \mathrm{~g}$

This SRM is chemically modified microparticulate silica intended for the calibration of instruments used to measure total carbon.

SRM

1216

Description

Carbon Modified Silica

$\begin{array}{ll}\text { Bottle } & \text { Mass Fraction (\%) } \\ \text { I } & 0.70 \\ \text { II } & 9.06 \\ \text { III } & 17.04\end{array}$

\section{Used Auto Catalysts}

Unit Size: $70 \mathrm{~g}$

$\begin{array}{lll}\text { SRM } & \text { Description } & \text { Elemental Composition } \\ 2557 & \text { Recycled Monolith } & \\ 2556 & \text { Recycled Pellet } & \mathrm{Pt}, \mathrm{Pd}, \mathrm{Rh}, \mathrm{Pb}\end{array}$

\section{Primary Gas Mixtures}

These SRMs are supplied in a DOT 3AL specification aluminum (6061 alloy) cylinder with a nominal pressure exceeding $12.4 \mathrm{MPa}$ that provides the user with approximately $0.73 \mathrm{~m}^{3}$ of usable mixture.

SRM

Nominal Amount-of-Substance $(\mu \mathrm{mol} / \mathrm{mol})$

Ambient Non-Methane Organics in Nitrogen

(15 components in large cylinder)

1800

$5 \mathrm{nmol} / \mathrm{mol}$

Carbon Dioxide in Air

(Certified for $\mathrm{CO}_{2}$ )

$1671 a$

340

$1672 a$

350

1676

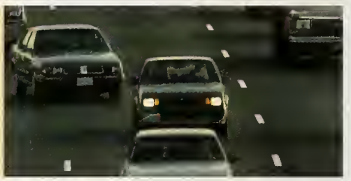

365 
Primary Gas Mixtures (continued)

SRM

Nominal Amount of Substance Fraction ( $\mu \mathrm{mol} / \mathrm{mol}$ )

Carbon Monoxide in Air

(Certified for $\mathrm{CO}$ )

$\begin{array}{ll}2612 a & 10 \\ 2613 a & 20 \\ 2614 a & 45\end{array}$

Carbon Dioxide in Nitrogen

(Certified for $\mathrm{CO}_{2}$ )

$\begin{array}{lc}1674 b^{*} & 7 \mathrm{~mol} \% \\ 1675 b * & 14 \mathrm{~mol} \% \\ 2619 a & 0.5 \mathrm{~mol} \% \\ 2620 a & 1.0 \mathrm{~mol} \% \\ 2621 a & 1.5 \mathrm{~mol} \% \\ 2622 a & 2.0 \mathrm{~mol} \% \\ 2623 a & 2.5 \mathrm{~mol} \% \\ 2624 a & 3.0 \mathrm{~mol} \% \\ 2625 a * & 3.5 \mathrm{~mol} \% \\ 2626 a & 4.0 \mathrm{~mol} \% \\ 2745 * & 16 \mathrm{~mol} \%\end{array}$

Carbon Monoxide in Nitrogen

(Certified for CO)

$\begin{array}{ll}1677 \mathrm{c} * & 10 \\ 1678 \mathrm{c} * & 50 \\ 1679 \mathrm{c} * & 100 \\ 1680 \mathrm{~b} * & 500 \\ 1681 \mathrm{~b} * & 1000 \\ 2635 \mathrm{a} * & 25 \\ 2636 \mathrm{a} * & 250 \\ 2637 \mathrm{a} * & 2500 \\ 2638 \mathrm{a} * & 5000 \\ 2639 a & 1 \mathrm{~mol} \% \\ 2640 a & 2 \mathrm{~mol} \mathrm{\%} \\ 2641 \mathrm{a} & 4 \mathrm{~mol} \% \\ 2642 a * & 8 \mathrm{~mol} \%\end{array}$

*Available as a NIST Traceable Reference Material (NTRM); from commercial suppliers.

(continued)

A suppliers list is available on our website. 
Primary Gas Mixtures (continued)

SRM

Carbon Monoxide in Nitrogen

(Certified for $\mathrm{CO}$ ) continued

$2740 a$

$2741 a$

Hydrogen Sulfide in Nitrogen

(Certified for $\mathrm{H}_{2} \mathrm{~S}$ )

2730

2731

Methane in Air

(Certified for $\mathrm{CH}_{4}$ )

$1658 a$

$1659 a$

1660a (also certified for $\mathrm{C}_{3} \mathrm{H}_{8}$ )

2750

2751

Nitric Oxide in Nitrogen

(Certified for NO)

$1683 b *$

$1684 b *$

$1685 b *$

$1686 b^{*}$

$1687 \mathrm{~b} *$

2629a*

2630*

2631a*

2735

$2736 a$

Oxides of Nitrogen in Air

(Certified for $\mathrm{NO}_{x}$ )

2660a*
Nominal Amount of Substance Fraction ( $\mu \mathrm{mol} / \mathrm{mol}$ )

$10 \mathrm{~mol} \mathrm{\%}$

$13 \mathrm{~mol} \%$

5

20

1

10

4 (methane)

1 (propane)

50

100

50

100

250

500

1000

20

1500

3000

800

2000

100

*Available as a NIST Traceable Reference Material (NTRM); from commercial suppliers.

(continued) A suppliers list is available on our website. 
Primary Gas Mixtures (continued)

SRM

Oxygen in Nitrogen (Certified for $\mathrm{O}_{2}$ )

2657a*

2658a*

2659a*

Propane in Air (Certified for $\mathrm{CH}_{4}$ )

$\begin{array}{ll}1660 a & 4 \text { (methane) } \\ \left.\text { (also certified for } \mathrm{C}_{3} \mathrm{H}_{8}\right) & 1 \text { (propane) } \\ 1666 \mathrm{~b} & 10 \\ 1667 \mathrm{~b} & 50 \\ 1668 \mathrm{~b} * & 100 \\ 1669 \mathrm{~b} & 500 \\ 2764 & 0.25\end{array}$

Propane in Nitrogen (Certified for $\mathrm{C}_{3} \mathrm{H}_{8}$ )

$2643 a$

$2644 a$

$2645 a$

$2646 a$

$2647 a$

$2648 a$

Sulfur Dioxide in Nitrogen (Certified for $\mathrm{SO}_{2}$ )

$\begin{array}{ll}1661 a * & 500 \\ 1662 a * & 1000 \\ 1663 a * & 1500 \\ 1664 a * & 2500 \\ 1693 a * & 50 \\ 1694 a * & 100 \\ 1696 a * & 3500\end{array}$

100

250

Nominal Amount of substance Fraction ( $\mu \mathrm{mol} / \mathrm{mol}$ )

$2 \mathrm{~mol} \%$

$10 \mathrm{~mol} \%$

$21 \mathrm{~mol} \%$

500

1000

2500

5000

3500

*Available as a NIST Traceable Reference Material (NTRM); from commercial suppliers. A suppliers list is available on our website.

The gas NTRM program was established in 1992 in partnership with the U.S. EPA and specialty gas companies as a means for providing end users with the wide variety of certified gas standards needed to implement the Emissions Trading Provision of the 1990 Clean Air Act. 


\author{
FOSSIL FUELS \\ Metal Constituents in Fossil Fuels

\begin{tabular}{|c|c|}
\hline SRM & $\mathrm{Pb}$ Concentration \\
\hline 2713 & Lead in Reference Fuels (19.4 $\mu \mathrm{g} / \mathrm{g} \mathrm{Pb})$ \\
\hline 2714 & Lead in Reference Fuels $(28.1 \mu \mathrm{g} / \mathrm{g} \mathrm{Pb})$ \\
\hline $1634 c$ & Trace Elements in Fuel Oil "No. 6" (As, Co, Ni, Pb, S, Se, V) \\
\hline RM 8505 & Vanadium in Crude Oil \\
\hline
\end{tabular}

\section{Unit Size}

$6 \times 20 \mathrm{~mL}$

$6 \times 20 \mathrm{~mL}$

$100 \mathrm{~mL}$

$250 \mathrm{~mL}$

\section{High Purity Liquids for Fuel Rating}

Unit Size: $100 \mathrm{~mL}$

$\begin{array}{lll}\text { SRM } & \text { Description } & \text { Purity (\%) } \\ 1816 \mathrm{a} & \text { Isooctane }(2,2,4-\text { Trimethylpentane) } & 99.987 \\ 1815 \mathrm{a} & \mathrm{n} \text {-Heptane } & 99.987\end{array}$

\section{Trace Elements in Coals and Coke}

$\begin{array}{lll}\text { SRM } & \text { Description } & \text { Unit Size (g) } \\ 2719 & \text { Calcined Petroleum Coke } & 50 \\ 1632 \mathrm{c} & \text { Coal (Bituminous) } & 50 \\ 1635 & \text { Coal (Subbituminous) } & 75 \\ 1633 \mathrm{~b} & \text { Coal Fly Ash } & 75 \\ 2689 & \text { Coal Fly Ash } & 3 \times 10 \mathrm{~g} \\ 2690 & \text { Coal Fly Ash } & 3 \times 10 \mathrm{~g} \\ 2691 & \text { Coal Fly Ash } & 3 \times 10 \mathrm{~g} \\ 2718 & \text { Green Petroleum Coke } & 50\end{array}$


Alcohols and Ethers [0xygenates] in Reference Fuels
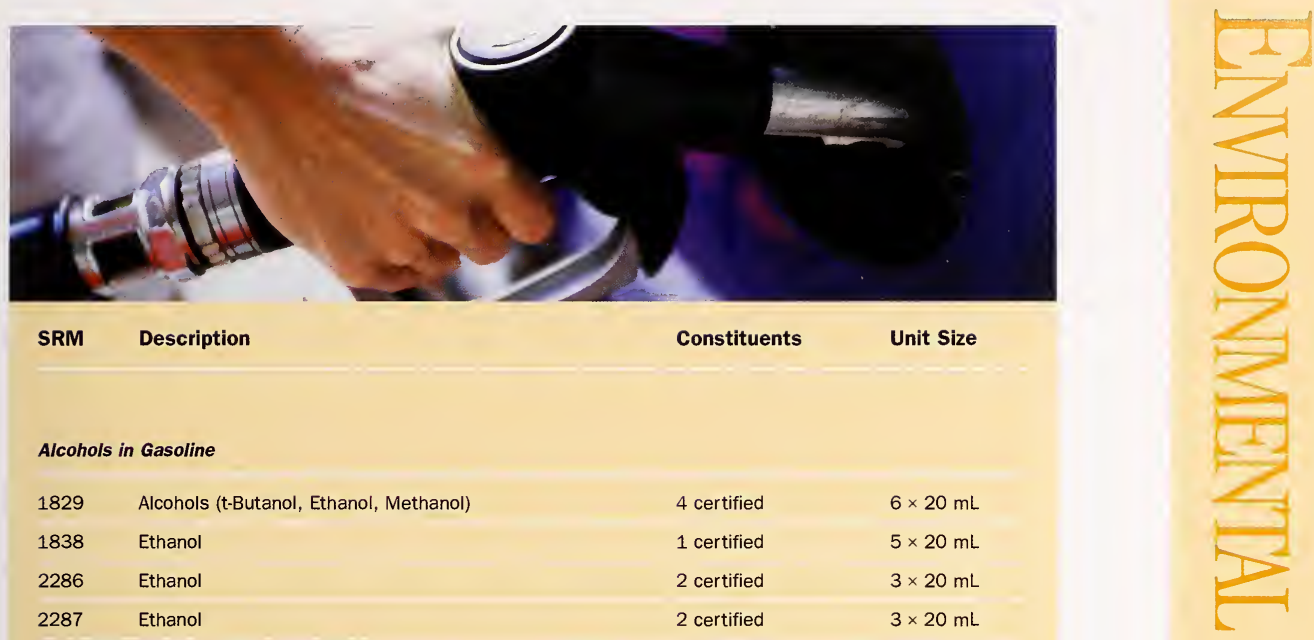

Alcohols in Gasoline

$\begin{array}{llll}1829 & \text { Alcohols (t-Butanol, Ethanol, Methanol) } & 4 \text { certified } & 6 \times 20 \mathrm{~mL} \\ 1838 & \text { Ethanol } & 1 \text { certified } & 5 \times 20 \mathrm{~mL} \\ 2286 & \text { Ethanol } & 2 \text { certified } & 3 \times 20 \mathrm{~mL} \\ 2287 & \text { Ethanol } & 2 \text { certified } & 3 \times 20 \mathrm{~mL} \\ 1839 & \text { Methanol } & 1 \text { certified } & 5 \times 20 \mathrm{~mL} \\ 1837 & \text { Methanol } & 2 \text { certified } & 5 \times 20 \mathrm{~mL}\end{array}$

Ethers in Gasoline

Unit Size: $3 \times 20 \mathrm{~mL}$

$\begin{array}{lll}2288 & \text { t-Amyl Methyl Ether } & 2 \text { certified } \\ 2289 & \text { t-Amyl Methyl Ether } & 2 \text { certified } \\ 2290 & \text { Ethyl t-Butyl Ether ETBE } & 2 \text { certified } \\ 2291 & \text { Ethyl t-Butyl Ether ETBE } & 2 \text { certified } \\ 2292 & \text { Methyl t-Butyl Ether MTBE } & 2 \text { certified } \\ 2293 & \text { Methyl t-Butyl Ether MTBE } & 2 \text { certified }\end{array}$

\section{Ethers and Ethanol in Reformulated Gasoline} Unit Size: $2 \times 20 \mathrm{~mL}$

$\begin{array}{lll}2294 & 11 \% \text { MTBE } & 4 \text { certified } \\ 2295 & 15 \% \text { MTBE } & 26 \text { reference } \\ & & 4 \text { certified } \\ 2296 & 13 \% \text { ETBE } & 26 \text { reference } \\ & & 4 \text { certified } \\ 2297 & 10 \% \text { Ethanol } & 26 \text { reference } \\ & & 4 \text { certified } \\ & & 26 \text { reference }\end{array}$




\section{Sulfur in Fossil Fuels}

SRM

\section{Coke Foundry \\ Unit Size: $50 \mathrm{~g}$}

2775 Foundry Coke

2776 Foundry Coke

0.825

Diesel Fuel Oil

Unit Size: $10 \times 10 \mathrm{~mL}$

2724b Sulfur in Diesel Fuel Oil

\section{Gasolines}

Unit Size: $2 \times 20 \mathrm{~mL}$ (unless otherwise noted)

2299 Gasoline (High Octane) $(5 \times 20 \mathrm{~mL})$ (In Prep)

2298 Reformulated Gasoline $(5 \times 20 \mathrm{~mL}$ ) (In Prep)

2294 Reformulated Gasoline (11\% MTBE)

0.00409

2295 Reformulated Gasoline (15\% MTBE)

0.0308

2296 Reformulated Gasoline (nominal $13 \%$ ETBE)

0.00400

2297

Reformulated Gasoline (nominal $10 \%$ Ethanol)

0.03037

\section{Kerosine}

Unit Size: $100 \mathrm{~mL}$

1616a Sulfur in Kerosine

0.01462

$1617 a$ Sulfur in Kerosine

0.17307

\section{Petroleum Coke}

Unit Size: $50 \mathrm{~g}$

2719 Trace Elements in Calcined Petroleum Coke

0.8877

2718 Trace Elements in Green Petroleum Coke

4.7032

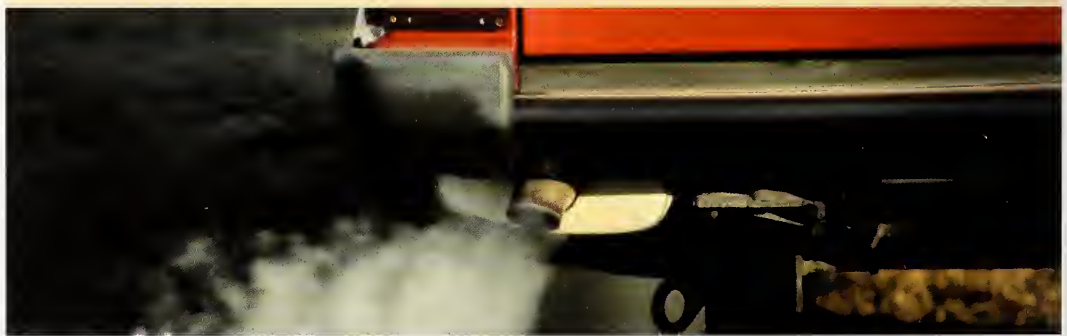


Sulfur in Fossil Fuels (continued)

\begin{abstract}
Residual Fuel Oil
Unit Size: $100 \mathrm{~mL}$

1619b Sulfur in Residual Fuel Oil

1620c Sulfur in Residual Fuel Oil

1621e Sulfur in Residual Fuel Oil

1622e Sulfur in Residual Fuel Oil

1623c Sulfur in Residual Fuel Oil

2717a Sulfur in Residual Fuel Oil

SRM Description
\end{abstract}

\section{Crude Oil}

Unit Size: $5 \times 10 \mathrm{~mL}$

2721 Crude Oil

2722 Crude Oil

\section{Coals}

Unit Size: $50 \mathrm{~g}$ (unless otherwise noted)

$\begin{array}{ll}\text { 2683b } & \text { Sulfur and Mercury in Coal } \\ 2684 \mathrm{~b} & \text { Sulfur and Mercury in Coal } \\ 2685 \mathrm{~b} & \text { Sulfur and Mercury in Coal } \\ 2692 \mathrm{~b} & \text { Sulfur and Mercury in Coal } \\ 2682 \mathrm{~b} & \text { Sulfur and Mercury in Coal (Subbituminous) } \\ 1632 \mathrm{c} & \text { Trace Elements in Coal Bituminous } \\ 1635 & \text { Trace Elements in Coal (Subbituminous) (75 g) }\end{array}$

0.6960

4.561

0.9480

2.1468

0.3806

2.9957

$\% \mathrm{~S}$

$\mathrm{Hg}(\mu \mathrm{g} / \mathrm{kg})$

$\begin{array}{ll}1.5832 & 0.0525 \\ 0.21037 & 0.1441\end{array}$

1.95590 .0

$3.076 \quad 97.4$

$4.730 \quad 146.2$

$1.170 \quad 133.3$

$0.4917 \quad 108.8$

$1.462 \quad 93.8$

$0.3616 \quad 10.9$

\section{Moisture in Oils and Alcohols}

$\begin{array}{ll}\text { SRM } & \text { Description } \\ \text { RM 8509 } & \text { Methanol } \\ \text { RM 8507 } & \text { Mineral Oil } \\ \text { RM 8510 } & \text { Moisture in Methanol } \\ \text { RM 8506a } & \text { Transformer Oil } \\ 2890 & \text { Water Saturated 1-Octanol }\end{array}$

Unit Size $(\mathrm{mL})$

$5 \mathrm{~mL}$

$10 \mathrm{~mL}$

$5 \mathrm{~mL}$

$5 \times 9.5 \mathrm{~mL}$

$5 \times 2 \mathrm{~mL}$ 


\section{GEOLOGICAL MATERIALS AND ORES}

\section{Ores}

$M|N| N G$

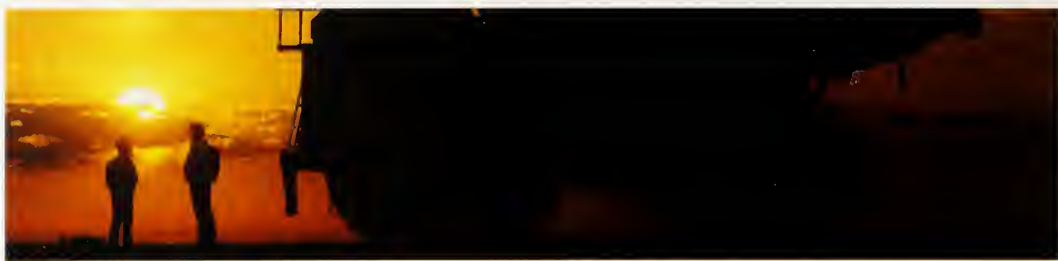

SRM Description

699 Alumina (Reduction Grade)

69b Bauxite, Arkansas

697 Bauxite, Dominican

698 Bauxite, Jamaican

696 Bauxite, Surinam

1835 Borate Ore

330 Copper Ore Mill Heads

331 Copper Ore Mill Tails

79a Fluorspar, Customs Grade

180 Fluorspar, High Grade

886 Gold Ore, Refractory

670 Iron Ore, Canada

690 Iron Ore, Canada

692 Iron Ore, Labrador

693 Iron Ore, Nimba

691 Iron Oxide, Reduced

182 Lithium Ore (Petalite)

181 Lithium Ore (Spodumene)

25d Manganese Ore

120c Phosphate Rock, Florida

694 Phosphate Rock, Western

600 Rutile Ore

2430 Scheelite Ore

277 Tungsten Concentrate

113b Zinc Concentrate
Unit Size (g)

60

60

60

60

60

60

100

100

120

120

200

90

100

100

100

100

45

45

60

90

90

90

100

45

100 


\section{Ore Bioleaching Substrate}

This RM is for use as a bioleaching substrate and for testing bioleaching rates.

RM Description

Unit Size (g)

8455 Pyrite Ore

100

\section{Chinese Ores}

Unit Size: $100 \mathrm{~g}$

These RMs are a well characterized series of skarn deposit ores developed and certified by the Hubei Geological Research Laboratory, Hubei Province, China.

\begin{tabular}{ll} 
RM & Description \\
8600 & Copper \\
8601 & Copper \\
8602 & Lead \\
8603 & Lead \\
8605 & Molybdenum \\
8606 & Molybdenum \\
8607 & Tungsten \\
8608 & Tungsten \\
8604 & Zinc \\
\hline
\end{tabular}

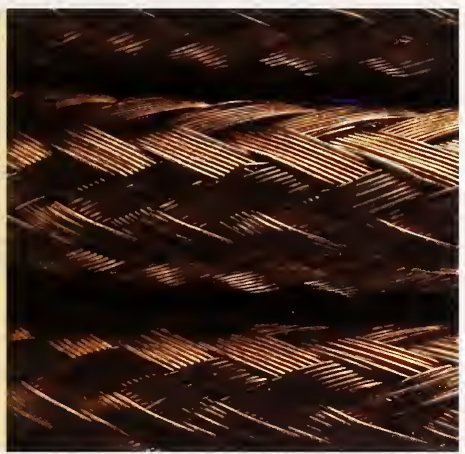

COPPER WIRE

\section{Clays}

$\begin{array}{lll}\text { SRM } & \text { Description } & \text { Unit Size (g) } \\ 679 & \text { Brick Clay } & 75 \\ 97 \mathrm{~b} & \text { Flint Clay } & 60 \\ 98 \mathrm{~b} & \text { Plastic Clay } & 60\end{array}$




\section{Rocks and Minerals}

$\begin{array}{lll}\text { SRM } & \text { Description } & \text { Unit Size (g) } \\ 688 & \text { Basalt Rock } & 60 \\ 70 a & \text { Feldspar, Potash } & 40 \\ 99 a & \text { Feldspar, Soda } & 40 \\ 81 a & \text { Glass Sand } & 75 \\ 165 a & \text { Glass Sand (Low Iron) } & 75 \\ 1413 & \text { Glass Sand (High Alumina) } & 75 \\ 1 c & \text { Limestone, Argillaceous } & 50 \\ 88 \mathrm{~b} & \text { Limestone, Dolomite } & 75 \\ 278 & \text { Obsidian Rock } & 35\end{array}$

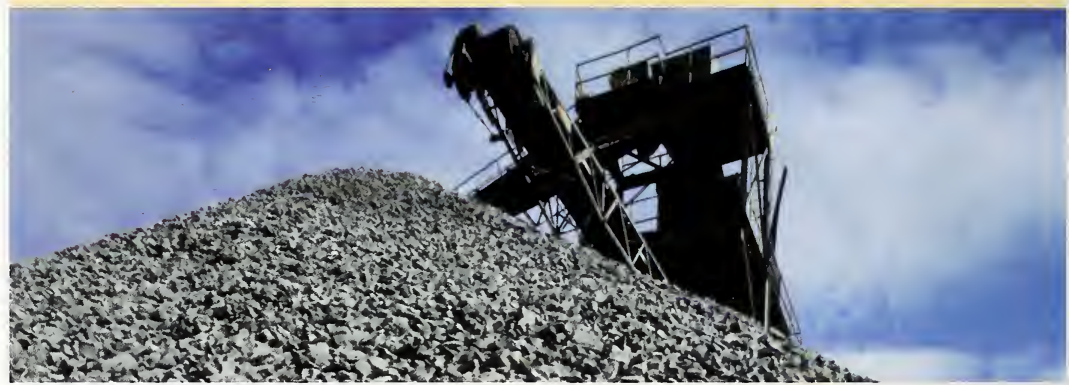

\section{Refractories}

$\begin{array}{llr}\text { SRM } & \text { Description } & \text { U } \\ 76 a & \text { Burnt Refractory (Al203-40\%) } & 75 \\ 77 a & \text { Burnt Refractory (Al203-60\%) } & 75 \\ 78 a & \text { Burnt Refractory (Al203-70\%) } & 75 \\ 198 & \text { Silica Brick } & 45 \\ 199 & \text { Silica Brick } & 45 \\ 154 b & \text { Titanium Dioxide } & 9\end{array}$




\section{MICROANALYSIS}

\section{Metals}

$\begin{array}{lll}\text { SRM } & \text { Description } & \text { Unit Size } \\ 482 & \text { Gold-Copper Wires for Microprobe Analysis } & \text { wires: } 6 \\ 481 & \text { Gold-Silver Wires for Microprobe Analysis } & \text { wires: } 6 \\ 480 & \text { Tungsten-20\% Molybdenum Alloy Electron Microprobe Standard } & \text { rod: } 1\end{array}$

\section{Synthetic Glasses}

$\begin{array}{lll}\text { SRM } & \text { Description } & \text { Unit Size } \\ 1873 & \begin{array}{l}\text { Barium-Zinc-Silicate Glasses for Microanalysis } \\ (\mathrm{K}-458, \mathrm{~K}-489, \mathrm{~K}-963)\end{array} & \text { rod: } 2 \mathrm{~mm} \times 2 \mathrm{~mm} \times 20 \mathrm{~mm} \\ 2066 & \begin{array}{l}\text { Glass Microspheres } \\ (\mathrm{K}-411)\end{array} & \text { glass microspheres: } 50 \mathrm{mg} \\ 1872 & \begin{array}{l}\text { Lead-Germanate Glasses for Microanalysis } \\ (\mathrm{K}-453, \mathrm{~K}-491, \mathrm{~K} 968)\end{array} & \text { rod: } 2 \mathrm{~mm} \times 2 \mathrm{~mm} \times 20 \mathrm{~mm}\end{array}$

\section{Thin Film for Transmission Electron Microscope}

$\begin{array}{llll}\text { SRM } & \text { Description } & \text { Certified Element } & \text { Unit Size } \\ \text { 2063a } & \text { Microanalysis Thin Film Mineral Glass } & \text { Ar, Ca, Fe, Mg, O, Si } & 1 \text { glass film }\end{array}$

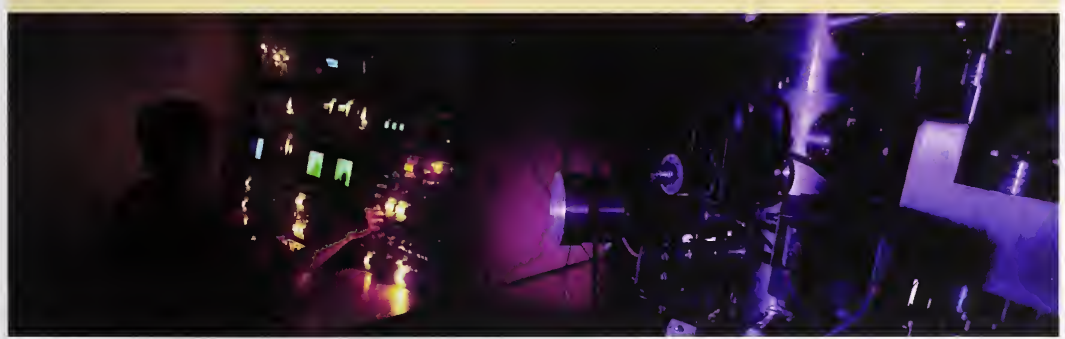

ELECTRON MICROSCOPE 


\section{ENGINE WEAR MATERIALS \\ Metallo-Organic Compounds}

Unit Size: $5 \mathrm{~g}$

These SRMs are for preparing solutions in oils of known and reproducible concentrations of metals.

\begin{tabular}{|c|c|c|}
\hline SRM & Description & Elemental Composition \\
\hline $1075 a$ & Aluminum 2-Ethylhexanoate & $8.07 \mathrm{Al}$ \\
\hline $1051 b$ & Barium Cyclohexanebutyrate & $28.7 \mathrm{Ba}$ \\
\hline $1080 a$ & Bis (1-phenyl-1,3-butanediono)copper (II) & $16.37 \mathrm{Cu}$ \\
\hline $1052 b$ & Bis(1-phenyl-1,3-butanediono)oxovanadium (IV) & $13.01 \mathrm{~V}$ \\
\hline $1053 a$ & Cadmium Cyclohexanebutyrate & $24.8 \mathrm{Cd}$ \\
\hline $1057 b$ & Dibutyltin bis (2-ethylhexanoate) (tin) & $22.95 \mathrm{Sn}$ \\
\hline $1059 c$ & Lead Cyclohexanebutyrate & $37.5 \mathrm{~Pb}$ \\
\hline $1060 a$ & Lithium Cyclohexanebutyrate & $4.1 \mathrm{Li}$ \\
\hline $1065 b$ & Nickel Cyclohexanebutyrate & $13.89 \mathrm{Ni}$ \\
\hline $1066 a$ & Octaphenylcyclotetrasiloxane & $14.14 \mathrm{Si}$ \\
\hline $1077 a$ & Silver 2-Ethylhexanoate & $42.60 \mathrm{Ag}$ \\
\hline $1069 b$ & Sodium Cyclohexanebutyrate & $12.0 \mathrm{Na}$ \\
\hline $1070 a$ & Strontium Cyclohexanebutyrate & $20.7 \mathrm{Sr}$ \\
\hline $1071 b$ & Triphenyl Phosphate & $9.48 P$ \\
\hline $1078 b$ & Tris (1-phenyl-1,3-butanediono)chromium (III) & $9.6 \mathrm{Cr}$ \\
\hline $1079 b$ & Tris (1-phenyl-1,3-butanediono)iron (III) & $10.45 \mathrm{Fe}$ \\
\hline $1073 b$ & Zinc Cyclohexanebutyrate & $16.66 \mathrm{Zn}$ \\
\hline
\end{tabular}




\section{Lubricating Base Oils}

These SRMs are for determining the concentrations of a single element in lubricating base oil. SRMs 1818a and 1819a consist of five bottles, approximately $20 \mathrm{~g}$ of liquid each; SRM 1836 consists of four sets of four ampoules, each ampoule containing approximately $4 \mathrm{~g}$ of liquid.

\begin{tabular}{lllllll} 
SRM & Description & \multicolumn{6}{l}{ Elemental Composition $\mathbf{( m g / k g )}$} \\
\cline { 3 - 7 } & & I & II & III & IV & V \\
1818 a & Total Chlorine & 31.6 & 60.0 & 78.2 & 154.4 & 234.0 \\
1836 & Total Nitrogen & 9.0 & 50.9 & 113.3 & 166.2 & \\
$1819 a$ & Total Sulfur & 423.5 & 741.1 & 4022 & 4689 & 6135
\end{tabular}

\section{Catalyst Characterization Material}

This RM is for determining the activity of FCC Catalysts by Microactivity Test and is distributed by NIST in cooperation with ASTM.
RM
Description
Unit Size
8590 High Sulfur Gas Oil Feed
$946 \mathrm{~mL}$

\section{Wear-Metals in Oil}

$\begin{array}{llc}\text { SRM } & \text { Description } & \text { Unit Size } \\ 1848 & \text { Lubricating Oil Additive Package } & 100 \mathrm{~mL} \\ 1084 \mathrm{a} & \text { Wear-Metals } & 5 \times 1.6 \mathrm{~g} \\ 1085 \mathrm{~b} & \text { Wear-Metals } & 5 \times 1.2 \mathrm{~g} \\ 1083 & \text { Wear-Metals (Base Oil) } & 150 \mathrm{~mL}\end{array}$




\section{HIGH PURITY MATERIALS}

39 Elemental Composition in High Purity Metals

40 Fine Gold Standards

40 Stoichlometric Standards

41 Microchemistry

42 Spectrometric Single Element Solutions

44 Anion Chromatography Solutions

44 Stable Isotopic Materials

45 Light Stable Isotopic Materials 


\section{Elemental Composition in High Purity Metals}

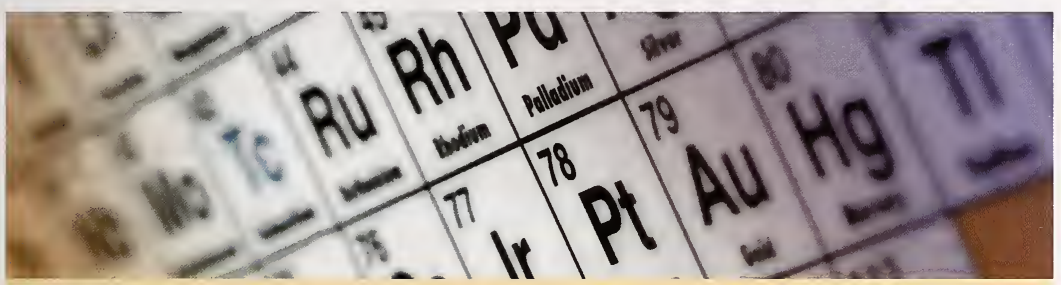

$\begin{array}{ll}\text { SRM } & \text { Description } \\ 685 \mathrm{R} & \text { High Purity Gold } \\ 685 \mathrm{~W} & \text { High Purity Gold } \\ 680 \mathrm{a}(\mathrm{L} 1) & \text { High Purity Platinum } \\ 680 \mathrm{a}(\mathrm{L} 2) & \text { High Purity Platinum } \\ 682 & \text { High Purity Zinc } \\ 885 & \text { Refined Copper } \\ 726 & \text { Selenium, Intermediate Purity } \\ 683 & \text { Zinc Metal } \\ 728 & \text { Zinc, Intermediate Purity }\end{array}$

\section{Unit Size}

$\begin{array}{ll}\text { rod: } & 5.9 \mathrm{~mm} \text { diameter } \times 25 \mathrm{~mm} \\ \text { wire: } & 1.4 \mathrm{~mm} \text { diameter } \times 102 \mathrm{~mm} \\ \text { wire: } & 0.51 \mathrm{~mm} \text { diameter } \times 10 \mathrm{~cm} \\ \text { wire: } & 0.51 \mathrm{~mm} \text { diameter } \times 1 \mathrm{~m} \\ \text { semicirc: } & 57 \mathrm{~mm} \\ \text { pin: } & 200 \mathrm{~g} \\ \text { shot: } & 450 \mathrm{~g} \\ \text { semicirc: } & 57 \mathrm{~mm} \\ \text { shot: } & 450 \mathrm{~g}\end{array}$
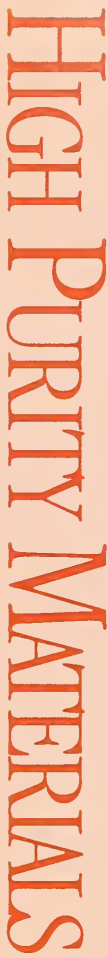


\section{Fine Gold Standards}

These RMs are a series of fine gold and gold bullion products developed and certified by the Royal Canadian Mint (RCM), Ottawa, Canada and distributed by NIST. The fine gold RMs are primarily intended for use as calibration standards for the determination of trace elements by solid sample spectrometric methods; the gold bullion RMs are primarily intended for use as quality control check standards for fire assay. There are five sets of RMs in the gold bullion series (RMs 8068-8082) available in three forms: disc ( $25 \mathrm{~mm}$ diameter $\times 20 \mathrm{~mm}$ ); wire ( $2 \mathrm{~mm}$ diameter); and foil $(35 \mathrm{~mm} \times 40 \mathrm{~mm} \times 1 \mathrm{~mm})$. There are six sets of RMs in the fine gold series (RMs 8050-8067) available in three forms: block $(25 \mathrm{~mm} \times 25 \mathrm{~mm} \times 2.5 \mathrm{~mm})$; wire ( $2 \mathrm{~mm}$ diameter $)$; and turnings $(25 \mathrm{~g})$.

\section{Stoichiometric Standards}

These SRMs are defined as primary, working, and secondary standards in accordance with recommendations of the Analytical Chemistry Section of the International Union of Pure and Applied Chemistry [Ref. Analyst 90, 251 (1965)]. These definitions are as follows:

- Primary Standard: a commercially available substance of purity $100 \% \pm 0.02 \%$ (Purity $99.98+\%$ )

- Working Standard: a commercially available substance of purity $100 \% \pm 0.05 \%$ (Purity 99. 95+ \%)

- Secondary Standard: a substance of lower purity which can be standardized against a primary grade standard

\begin{tabular}{|c|c|c|c|c|}
\hline SRM & Description & Certified Use & $\begin{array}{l}\text { Stoichiometric } \\
\text { Purity }(\%)\end{array}$ & Unit Size (g) \\
\hline 951 & Boric Acid & $\begin{array}{l}\text { Acidimetric and Boron } \\
\text { Isotopic Value }\end{array}$ & 100.00 & 100 \\
\hline $84 \mathrm{k}$ & Potassium Hydrogen Phthalate & Acidimetric Standard & 99.9911 & 60 \\
\hline $350 a$ & Benzoic Acid & Acidimetric Standard & 99.9958 & 30 \\
\hline 351 & Sodium Carbonate & Acidimetric Standard & 99.9796 & 50 \\
\hline $723 d$ & $\begin{array}{l}\text { Tris(hydroxymethyl)aminomethane } \\
\text { In Prep }\end{array}$ & Acidimetric Standard & - & - \\
\hline 987 & Strontium Carbonate & Assay and Isotopic Values & 99.98 & 1 \\
\hline $999 a$ & Potassium Chloride & $\begin{array}{l}\text { Assay Values for: } \\
\text { 1. Potassium Chloride } \\
\text { 2. Postassium } \\
\text { 3. Chloride }\end{array}$ & $\begin{array}{l}99.9817 \\
52.4354 \\
47.5463\end{array}$ & 60 \\
\hline $136 \mathrm{e}$ & Potassium Dichromate & Oxidimetric Standard & 99.984 & 60 \\
\hline $17 \mathrm{e}$ & Sucrose & Polarimetric Standard & 99.950 & 60 \\
\hline $917 b$ & D-Glucose (Dextrose) & Polarimetric Standard & 99.7 & 50 \\
\hline $40 \mathrm{~h}$ & Sodium Oxalate & Reductometric Standard & 99.972 & 60 \\
\hline $83 d$ & Arsenic Trioxide & Reductometric Standard & 99.9926 & 60 \\
\hline
\end{tabular}




\section{Microchemistry}

Unit Size: $2 g$
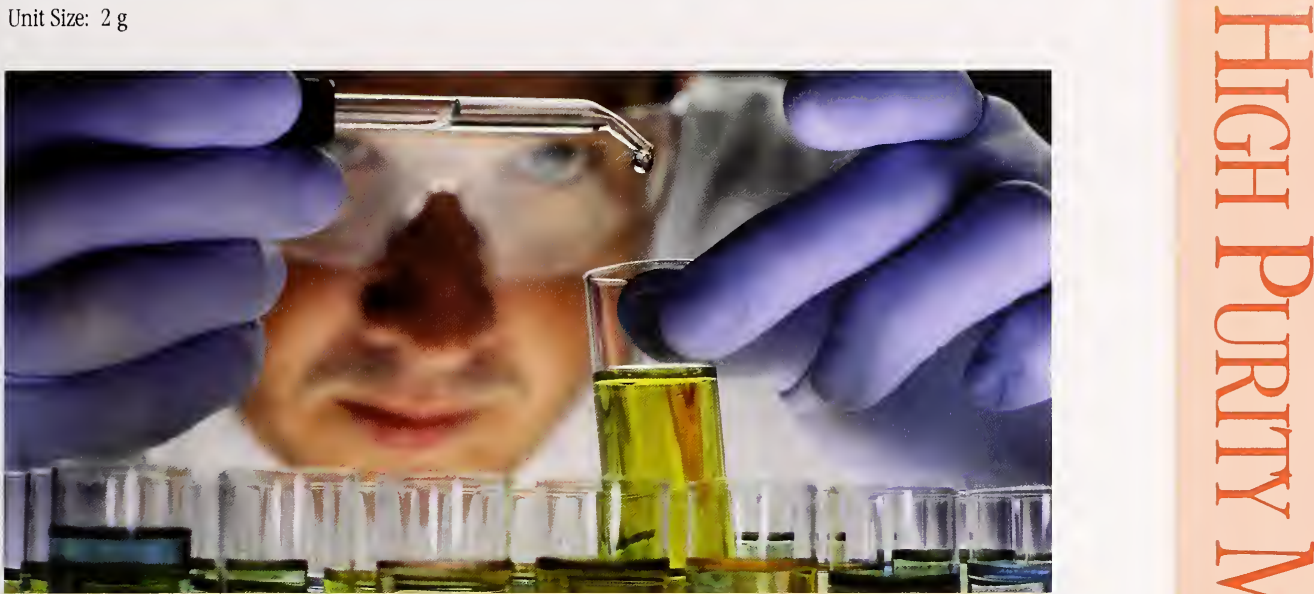

SRM Description

Certified Component

$\begin{array}{lll}141 \mathrm{~d} & \text { Acetanilide } & \mathrm{C}, \mathrm{H}, \mathrm{N}, \mathrm{O} \\ 142 & \text { Anisic Acid } & \mathrm{CH}_{3} \mathrm{O}- \\ 143 \mathrm{~d} & \text { Cystine } & \mathrm{C}, \mathrm{H}, \mathrm{N}, \mathrm{S}, \mathrm{O} \\ 2144 & \text { m-Chlorobenzoic Acid } & \mathrm{Cl} \\ 148 & \text { Nicotinic Acid } & \mathrm{C}, \mathrm{H}, \mathrm{N} \\ 2143 & \text { p-Flurobenzoic Acid } & \mathrm{F} \\ 2141 & \text { Urea } & \mathrm{N}\end{array}$




\section{Spectrometric Single Element Solutions}

Unit Size: $50 \mathrm{~mL}$

These SRMs are intended as standard solutions for use in calibrating instruments used in atomic spectrometry, including atomic absorption spectrometry, inductively coupled plasma optical spectrometry, and inductively coupled plasma mass spectrometry.

\begin{tabular}{|c|c|c|}
\hline SRM & Element & Nominal Acid Concentration \\
\hline 3101a & Aluminum & $\mathrm{HNO}_{3} 10 \%$ \\
\hline $3102 a$ & Antimony & $\mathrm{HNO}_{3} 10 \%+\mathrm{HF} 2 \%$ \\
\hline $3103 a$ & Arsenic & $\mathrm{HNO}_{3} 15 \%$ \\
\hline $3104 a$ & Barium & $\mathrm{HNO}_{3} 1 \%$ \\
\hline $3105 a$ & Beryllium & $\mathrm{HNO}_{3} 10 \%$ \\
\hline 3106 & Bismuth & $\mathrm{HNO}_{3} 10 \%$ \\
\hline 3107 & Boron & $\mathrm{H}_{2} \mathrm{O}$ \\
\hline 3108 & Cadmium & $\mathrm{HNO}_{3} 10 \%$ \\
\hline $3109 a$ & Calcium & $\mathrm{HNO}_{3} 10 \%$ \\
\hline 3110 & Cerium & $\mathrm{HNO}_{3} 10 \%$ \\
\hline $3111 \mathrm{a}$ & Cesium & $\mathrm{HNO}_{3} 1 \%$ \\
\hline $3112 a$ & Chromium & $\mathrm{HNO}_{3} 10 \%$ \\
\hline 3113 & Cobalt & $\mathrm{HNO}_{3} 10 \%$ \\
\hline 3114 & Copper & $\mathrm{HNO}_{3} 10 \%$ \\
\hline $3115 a$ & Dysprosium & $\mathrm{HNO}_{3} 10 \%$ \\
\hline $3116 a$ & Erbium & $\mathrm{HNO}_{3} 10 \%$ \\
\hline $3117 a$ & Europium & $\mathrm{HNO}_{3} 16 \%$ \\
\hline $3118 a$ & Gadolinium & $\mathrm{HNO}_{3} 10 \%$ \\
\hline $3119 a$ & Gallium & $\mathrm{HNO}_{3} 10 \%$ \\
\hline $3120 a$ & Germanium & $\mathrm{HNO}_{3} 10 \%+\mathrm{HF} 2 \%$ \\
\hline 3121 & Gold & $\mathrm{HNO}_{3} 5 \%+\mathrm{HF} 2 \%$ \\
\hline 3122 & Hafnium & $\mathrm{HNO}_{3} 10 \%+\mathrm{HF} 2 \%$ \\
\hline 3123a & Holmium & $\mathrm{HNO}_{3} 16 \%$ \\
\hline $3124 a$ & Indium & $\mathrm{HNO}_{3} 10 \%$ \\
\hline $3126 a$ & Iron & $\mathrm{HNO}_{3} 10 \%$ \\
\hline $3127 a$ & Lanthanum & $\mathrm{HNO}_{3} 10 \%$ \\
\hline 3128 & Lead & $\mathrm{HNO}_{3} 10 \%$ \\
\hline $3129 a$ & Lithium & $\mathrm{HNO}_{3} 1 \%$ \\
\hline $3130 a$ & Lutetium & $\mathrm{HNO}_{3} 10 \%$ \\
\hline $3131 a$ & Magnesium & $\mathrm{HNO}_{3} 10 \%$ \\
\hline 3132 & Manganese & $\mathrm{HNO}_{3} 10 \%$ \\
\hline
\end{tabular}

(continued) 
Spectrometric Single Element Solutions (continued)

\begin{tabular}{|c|c|c|}
\hline SRM & Element & Nominal Acid Concentration \\
\hline 3133 & Mercury & $\mathrm{HNO}_{3} 10 \%$ \\
\hline 3134 & Molybdenum & $\mathrm{HCl} 10 \%$ \\
\hline $3135 a$ & Neodymium & $\mathrm{HNO}_{3} 10 \%$ \\
\hline 3136 & Nickel & $\mathrm{HNO}_{3} 10 \%$ \\
\hline 3137 & Niobium & $\mathrm{HNO}_{3} 10 \%+\mathrm{HF} 2 \%$ \\
\hline 3138 & Palladium & $\mathrm{HCl} 10 \%$ \\
\hline $3139 a$ & Phosphorus & $\mathrm{HNO}_{3} \mathrm{O} .8 \%$ \\
\hline 3140 & Platinum & $\mathrm{HCl} 10 \%$ \\
\hline $3141 a$ & Potassium & $\mathrm{HNO}_{3} 1 \%$ \\
\hline $3142 a$ & Praseodymium & $\mathrm{HNO}_{3} 10 \%$ \\
\hline 3143 & Rhenium & $\mathrm{HNO}_{3} 10 \%$ \\
\hline 3144 & Rhodium & $\mathrm{HCl} 10 \%$ \\
\hline $3145 a$ & Rubidium & $\mathrm{HNO}_{3} 1 \%$ \\
\hline $3147 a$ & Samarium & $\mathrm{HNO}_{3} 10 \%$ \\
\hline $3148 a$ & Scandium & $\mathrm{HNO}_{3} 10 \%$ \\
\hline 3149 & Selenium & $\mathrm{HNO}_{3} 10 \%$ \\
\hline 3150 & Silicon & $\mathrm{H}_{2} \mathrm{O}$ \\
\hline 3151 & Silver & $\mathrm{HNO}_{3} 10 \%$ \\
\hline $3152 a$ & Sodium & $\mathrm{HNO}_{3} 1 \%$ \\
\hline 3153a & Strontium & $\mathrm{HNO}_{3} 10 \%$ \\
\hline 3154 & Sulfur & $\mathrm{H}_{2} \mathrm{SO}_{4} 0.1 \%$ \\
\hline 3155 & Tantalum & $\mathrm{HNO}_{3} \quad 10 \%+\mathrm{HF} 2 \%$ \\
\hline 3156 & Tellurium & $\mathrm{HCl} 20 \%$ \\
\hline $3157 a$ & Terbium & $\mathrm{HNO}_{3} 16 \%$ \\
\hline 3158 & Thallium & $\mathrm{HNO}_{3} 10 \%$ \\
\hline 3159 & Thorium & $\mathrm{HNO}_{3} 10 \%$ \\
\hline $3160 a$ & Thulium & $\mathrm{HNO}_{3} 10 \%$ \\
\hline $3161 a$ & Tin & $\mathrm{HNO}_{3} 5 \%+\mathrm{HF} 2 \%$ \\
\hline $3162 a$ & Titanium & $\mathrm{HNO}_{3} 10 \%+\mathrm{HF} 2 \%$ \\
\hline 3163 & Tungsten & $\mathrm{HNO}_{3} 7 \%+\mathrm{HF} 4 \%$ \\
\hline 3164 & Uranium & $\mathrm{HNO}_{3} 10 \%$ \\
\hline 3165 & Vanadium & $\mathrm{HNO}_{3} 10 \%$ \\
\hline $3166 a$ & Ytterbium & $\mathrm{HNO}_{3} 16 \%$ \\
\hline $3167 a$ & Yttrium & $\mathrm{HNO}_{3} 10 \%$ \\
\hline $3168 a$ & Zinc & $\mathrm{HNO}_{3} 10 \%$ \\
\hline 3169 & Zirconium & $\mathrm{HNO}_{3} 10 \%+\mathrm{HF} 2 \%$ \\
\hline
\end{tabular}




\section{Anion Chromatography Solutions}

Unit Size: $50 \mathrm{~mL}$

These SRMs are single component solutions prepared gravimetrically for use in anion chromatography or any other technique that requires aqueous standard solutions for calibration of control materials.

$\begin{array}{ll}\text { SRM } & \text { Description } \\ 3184 & \text { Bromide (In Prep) } \\ 3182 & \text { Chloride } \\ 3183 & \text { Fluoride } \\ 3185 & \text { Nitrate } \\ 3186 & \text { Phosphate } \\ 3181 & \text { Sulfate }\end{array}$

\section{Stable Isotopic Materials}

\begin{tabular}{ll} 
SRM & Description \\
951 & Boron Isotope Standard \\
952 & Enriched ${ }^{10} \mathrm{~B}$ Isotope Standard \\
$975 a$ & Chlorine Isotope Standard \\
976 & Copper Isotope Standard \\
977 & Bromine Isotope Standard \\
978 a & Silver Isotope Standard \\
979 & Chromium Isotope Standard \\
980 & Magnesium Isotope Standard \\
981 & Lead Isotope Standard, Natural \\
982 & Lead Isotope Standard, \\
& 208Pb/206Pb Equal Atom \\
983 & Lead Isotope Standard, Radiogenic \\
984 & Rubidium Isotope Standard \\
985 & Potassium Isotope Standard \\
986 & Nickel Isotope Standard \\
987 & Strontium Isotope Standard \\
991 & Nitrate Spike Isotope Standard, ${ }^{206} \mathrm{~Pb}$ \\
994 & Gallium Isotope Standard \\
997 & Thallium Isotope Standard \\
\hline 9 &
\end{tabular}

Nominal Concentration $\mathbf{( m g / k g})$
1000
1000
1000
1000
1000
1000

$\begin{array}{ll}\text { Chemical Form } & \text { Unit Size (g) } \\ \text { Boric Acid } & 100 \\ \text { Boric Acid } & 0.25 \\ \text { Sodium Chloride } & 0.25 \\ \text { Metal } & \text { disk: } 0.4 \\ \text { Sodium Bromide } & 0.25 \\ \text { Silver Nitrate } & 0.25 \\ \text { Chromium Nitrate } & 0.25 \\ \text { Metal } & 0.25 \\ \text { Metal } & \text { wire: } 1.0 \\ \text { Metal } & \text { wire: } 1.0 \\ & \\ \text { Metal } & \text { wire: } 1.0 \\ \text { Rubidium Chloride } & 0.25 \\ \text { Potassium Chloride } & 1.0 \\ \text { Metal } & 0.5 \\ \text { Strontium Carbonate } & 1.0 \\ \text { Nitric Acid } & 15 \\ \text { Metal } & \text { disk: } 0.25 \\ \text { Metal } & \text { rod: } 0.25\end{array}$




\section{Light Stable Isotopic Materials}

These RMs are distributed by NIST on behalf of the International Atomic Energy Agency (IAEA). At the request of the IAEA, quantities of these materials are limited to one unit of each RM per laboratory every 3 years.

\begin{tabular}{|c|c|c|}
\hline \multirow[t]{4}{*}{ Isotopic Ratio Legend: } & 1. $\mathrm{D} / \mathrm{H}$ & 5. ${ }^{30} \mathrm{Si} /{ }^{28} \mathrm{Si}$ \\
\hline & 2. ${ }^{18} \mathrm{O} /{ }^{16} \mathrm{O}$ & 6. ${ }^{15} \mathrm{~N} /{ }^{14} \mathrm{~N}$ \\
\hline & 3. ${ }^{13} \mathrm{C} /{ }^{12} \mathrm{C}$ & 7. ${ }^{34} \mathrm{~S} /{ }^{32} \mathrm{~S}$ \\
\hline & 4. ${ }^{6} \mathrm{Li} /{ }^{7} \mathrm{Li}$ & \\
\hline
\end{tabular}

\begin{tabular}{|c|c|c|c|}
\hline $\mathbf{R M}$ & Description & Isotopic Ratios & Unit Size \\
\hline 8535 & VSMOW-Water & 1,2 & $20 \mathrm{~mL}$ \\
\hline 8536 & GISP-Water & 1,2 & $20 \mathrm{~mL}$ \\
\hline 8537 & SLAP-Water & 1,2 & $20 \mathrm{~mL}$ \\
\hline 8538 & NBS30-Biotite & $1,2,3$ & $2 g$ \\
\hline 8539 & NBS22-Oil & $1,2,3$ & $1 \mathrm{~mL}$ \\
\hline 8540 & PEFI-Polyethylene & $1,2,3$ & $\sim 2 \mathrm{mg}$ \\
\hline 8541 & USGS24-Graphite & $1,2,3$ & $0.8 \mathrm{~g}$ \\
\hline 8542 & Sucrose ANU-Sucrose & $1,2,3$ & $1 \mathrm{~g}$ \\
\hline 8543 & NBS18-Carbonatite & 2,3 & $0.4 \mathrm{~g}$ \\
\hline 8544 & NBS18-Limestone & 2,3 & $0.4 \mathrm{~g}$ \\
\hline 8545 & LSVEC-Lithium Carbonate & 3,4 & $0.4 \mathrm{~g}$ \\
\hline 8546 & NBS28-Silica Sand (0ptical) & 2,5 & $0.4 \mathrm{~g}$ \\
\hline 8547 & IAEA-N1-Ammonium Sulfate & 6 & $0.4 \mathrm{~g}$ \\
\hline 8548 & IAEA-N2-Ammonium Sulfate & 6 & $0.4 \mathrm{~g}$ \\
\hline 8549 & IAEA-N3-Potassium Nitrate & 6 & $0.4 \mathrm{~g}$ \\
\hline 8550 & USGS25-Ammonium Sulfate & 6 & $0.4 \mathrm{~g}$ \\
\hline 8551 & USGS26-Ammonium Sulfate & 6 & $0.4 \mathrm{~g}$ \\
\hline 8552 & NSVEC-Gaseous Nitrogen & 6 & $300 \mu \mathrm{mol}$ \\
\hline 8553 & Soufre de Lacq - Elemental Sulfur & 2,7 & $0.5 \mathrm{~g}$ \\
\hline 8554 & IAEA-S1-Silver Sulfide & 2,7 & $0.5 \mathrm{~g}$ \\
\hline 8555 & IAEA-S2-Silver Sulfide & 2,7 & $0.5 \mathrm{~g}$ \\
\hline 8556 & NBS123-Sphalerite & 2,7 & $0.5 \mathrm{~g}$ \\
\hline 8557 & NBS127-Barium Sulfate & 2,7 & $0.5 \mathrm{~g}$ \\
\hline 8558 & USGS32-Potassium Nitrate & 6 & $0.5 \mathrm{~g}$ \\
\hline 8562 & $\mathrm{CO}_{2}$-Heavy, Paleomarine Origin & 2,3 & 2 tubes: $9 \mathrm{~mm}$ diameter $\times 300 \mathrm{~mm}$ \\
\hline 8563 & $\mathrm{CO}_{2}$-Light, Petrochemical Origin & 2,3 & 2 tubes: $9 \mathrm{~mm}$ diameter $\times 300 \mathrm{~mm}$ \\
\hline 8564 & $\begin{array}{l}\mathrm{CO}_{2} \text {-Biogenic, Modern } \\
\text { Biomass Origin }\end{array}$ & 2,3 & 2 tubes: $9 \mathrm{~mm}$ diameter $\times 300 \mathrm{~mm}$ \\
\hline
\end{tabular}




\section{INDUSTRIAL MATERIAIS}

47 Ferrous Metals

56 Nonferrous Metals

61 Ceramics and Glasses

63 Cements

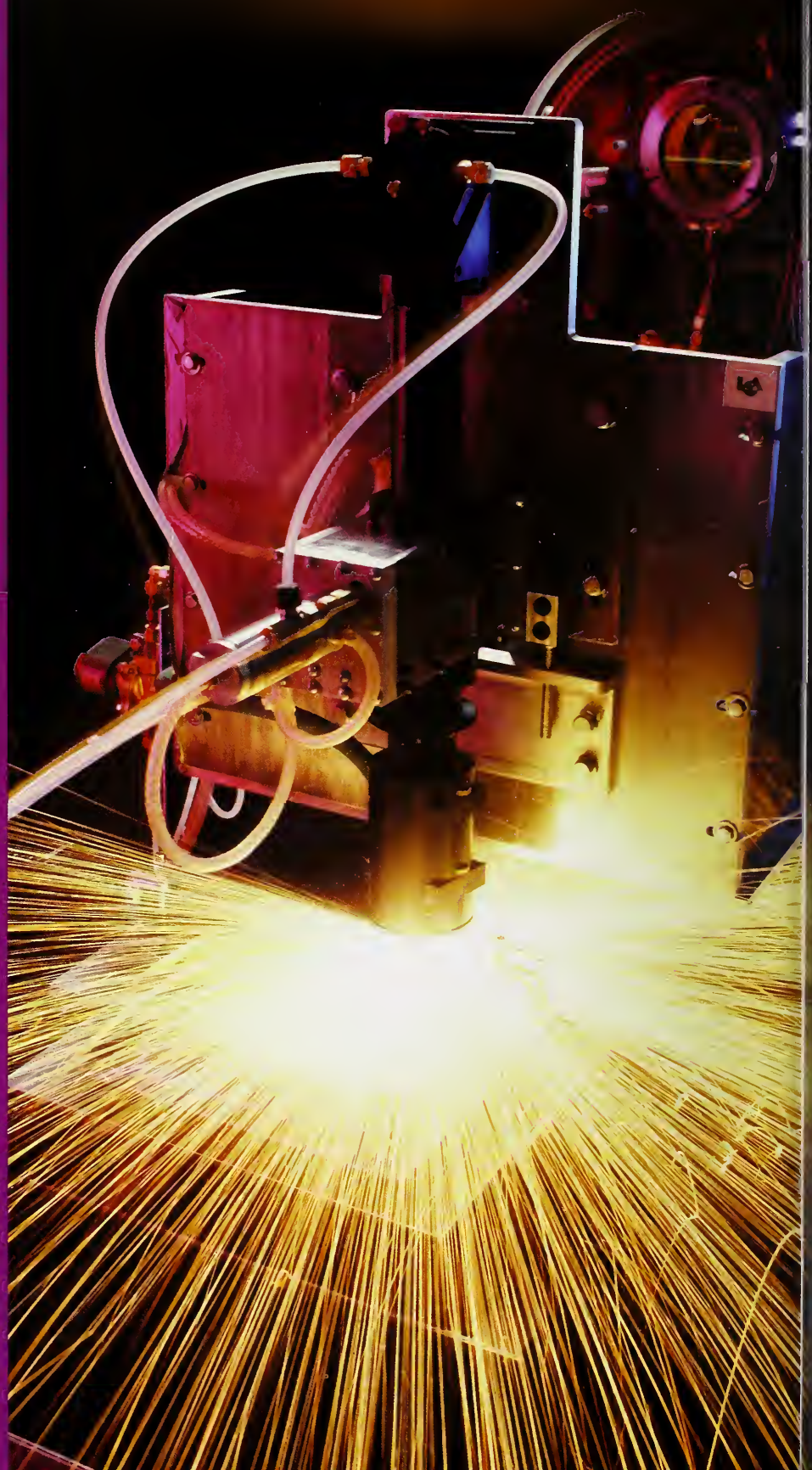




\section{FERROUS METALS}

\section{Steels}

These SRMs consist of selected steel alloys that provide a wide range of analytical values for relevant elements. Please visit our website to view the relevant certificate or report of investigation for all available certified and non-certified values. These RMs are a series of skarn deposit ores developed and certified by the Hubei Geological Research Laboratory, Hubei Province, China.

\section{Plain Carbon Steels (chip)}

Unit Size: $150 \mathrm{~g}$ (unless otherwise noted)

$\begin{array}{ll}\text { SRM } & \text { Description } \\ 178 & 0.4 \text { C Basic Oxygen Furnace Steel } \\ 13 \mathrm{~g} & 0.6 \% \text { Carbon Steel } \\ 20 \mathrm{~g} & \text { AISI } 1045 \text { Steel } \\ 14 \mathrm{~g} & \text { AISI } 1078 \text { Carbon Steel } \\ 368 & \text { AISI 1211 Steel } \\ 19 \mathrm{~h} & \text { Basic Electric Steel, 0.2 \% Carbon } \\ & \\ \text { Basic } 0 \text { pen-Hearth Steel } \\ 15 \mathrm{~h} & 0.1 \% \text { Carbon } \\ 11 \mathrm{~h} & 0.2 \% \text { Carbon } \\ 12 \mathrm{~h} & 0.4 \% \text { Carbon } \\ 152 \mathrm{a} & 0.5 \% \text { Carbon (Tin-Bearing) } \\ 337 \mathrm{a} & 1 \% \text { Carbon (300 g) } \\ \end{array}$
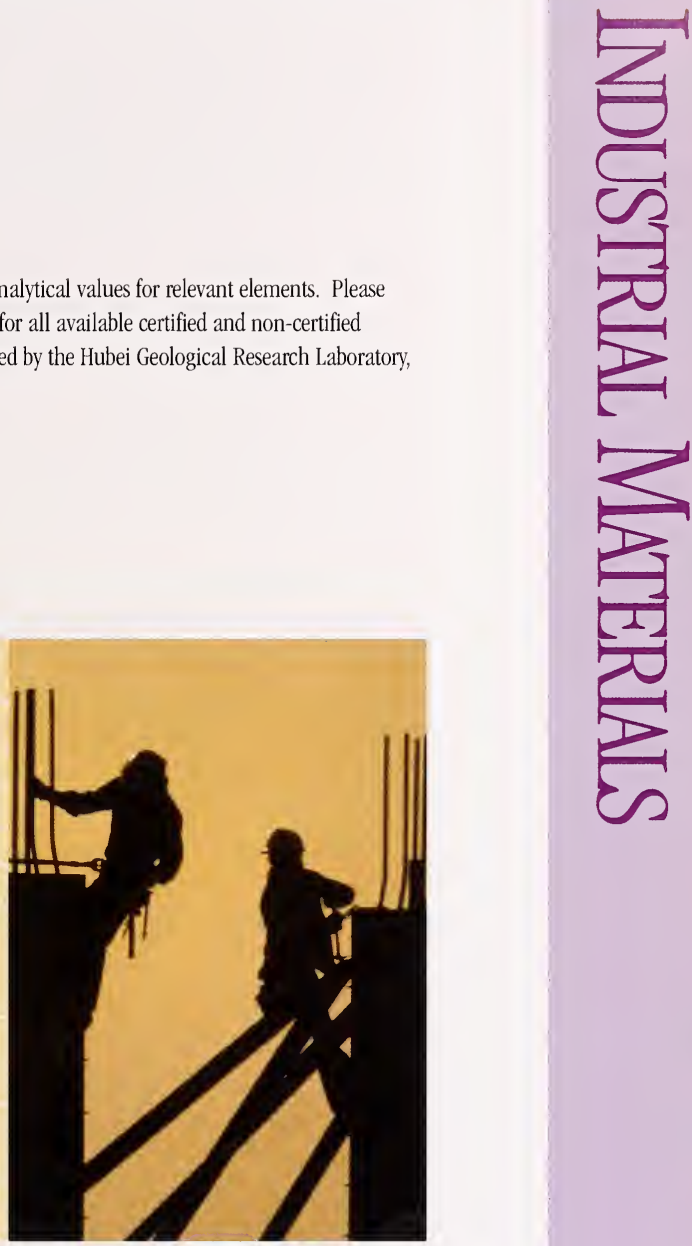


\section{Low Alloy Steels (disk and rod)}

Nominal Sizes for Solid Steel SRMs:

600 Series: $3.2 \mathrm{~mm}$ diameter $\times 51 \mathrm{~mm}$

1100 and 1200 Series: $31 \mathrm{~mm}$ diameter $\times 19 \mathrm{~mm}$

1700 Series: $34 \mathrm{~mm}$ diameter $\times 19 \mathrm{~mm}$

A "C" preceding the SRM number indicates a chill cast sample; $31 \mathrm{~mm}$ diameter $\times 19 \mathrm{~mm}$.

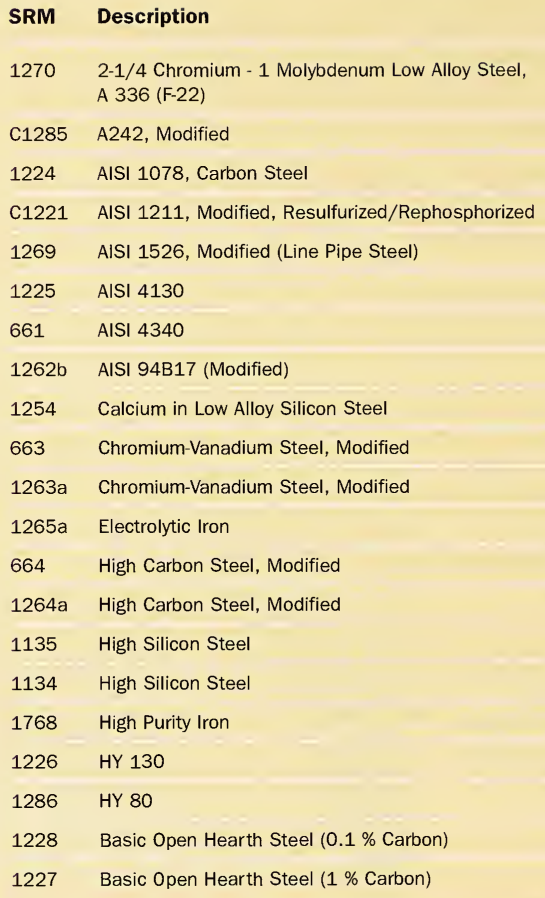


Low Alloy Steels (disk and rod) (continued)

$\begin{array}{ll}\text { SRM } & \text { Description } \\ 1761 & \text { Low Alloy Steel } \\ 1762 & \text { Low Alloy Steel } \\ 1763 & \text { Low Alloy Steel } \\ 1764 & \text { Low Alloy Steel } \\ 1765 & \text { Low Alloy Steel } \\ 1766 & \text { Low Alloy Steel } \\ 1767 & \text { Low Alloy Steel }\end{array}$

\section{Low Alloy Steels (chip)}

Unit Size: $150 \mathrm{~g}$ (unless otherwise noted)

\begin{tabular}{|c|c|}
\hline SRM & Description \\
\hline $72 \mathrm{~g}$ & AISI 4130 \\
\hline 293 & AISI $8620(\mathrm{Cr}-\mathrm{Ni}-\mathrm{Mo})$ \\
\hline $139 b$ & AISI $8640(\mathrm{Cr}-\mathrm{Ni}-\mathrm{Mo})$ \\
\hline 291 & ASTM A213 (Cr - Mo) \\
\hline 163 & Chromium Steel (100 g) \\
\hline $36 b$ & Chromium-Molybdenum Steel \\
\hline 155 & Chromium-Tungsten Steel \\
\hline $129 c$ & SAE 112 High Sulfur \\
\hline 2171 & HSLA $100(6 \mathrm{Ni}-\mathrm{Cr}-\mathrm{Cr}-\mathrm{Cu}-\mathrm{Mo})$ \\
\hline $106 b$ & Nitralloy ${ }^{\mathrm{TM}} \mathrm{G}(\mathrm{Cr}-\mathrm{Mo}-\mathrm{Al})$ \\
\hline $32 \mathrm{e}$ & SAE $3140(\mathrm{Ni}-\mathrm{Cr})$ \\
\hline $100 \mathrm{~b}$ & SAE $340(\mathrm{Mn})$ \\
\hline $33 \mathrm{e}$ & SAE 4820 (Ni) \\
\hline $30 f$ & SAE $6150(\mathrm{Cr}-\mathrm{V})$ \\
\hline \multicolumn{2}{|c|}{ Silicon Steels } \\
\hline 179 & High Silicon Steel \\
\hline $125 b$ & High Silicon Steel, Calcium-Bearing \\
\hline $131 f$ & Low Carbon Silicon Steel \\
\hline
\end{tabular}




\section{Special Low Alloy Steels (chip and pin)}

Unit Size: $150 \mathrm{~g}$ (unless otherwise noted)

$\begin{array}{ll}\text { SRM } & \text { Description } \\ 2159 & \text { Low Alloy Steel (pin - 200 g) } \\ 2160 & \text { Low Alloy Steel (pin - 200 g) } \\ 2166 & \text { Low Alloy Steel } \\ 2167 & \text { Low Alloy Steel } \\ 361 & \text { AISI 4340 Steel } \\ 362 & \text { AISI 94B17, Modified } \\ 363 & \text { Chromium-Vanadium Steel, Modified } \\ 364 & \text { High Carbon Steel, Modified } \\ 2168 & \text { High Purity Iron }\end{array}$

\section{High Alloy Steels (chip)}

Unit Size: $150 \mathrm{~g}$ (unless otherwise noted)

$\begin{array}{ll}\text { SRM } & \text { Description } \\ 345 a & \text { Cu Precipitation Hardening Steel }(15 \mathrm{Cr}-4 \mathrm{Ni}) \\ 344 & \text { Mo Precipitation Hardening Steel }(15 \mathrm{Cr}-7 \mathrm{Ni}) \\ 126 \mathrm{c} & \text { High Nickel Steel (36 \% Ni) } \\ 868 & \text { High Temperature Alloy (Fe-Ni-Co) (100 g) } \\ 348 \mathrm{a} & \text { High Temperature Alloy A286 (Ni-Cr) } \\ 862 & \text { High Temperature Alloy L605 (100 g) } \\ 346 a & \text { Valve Steel }\end{array}$

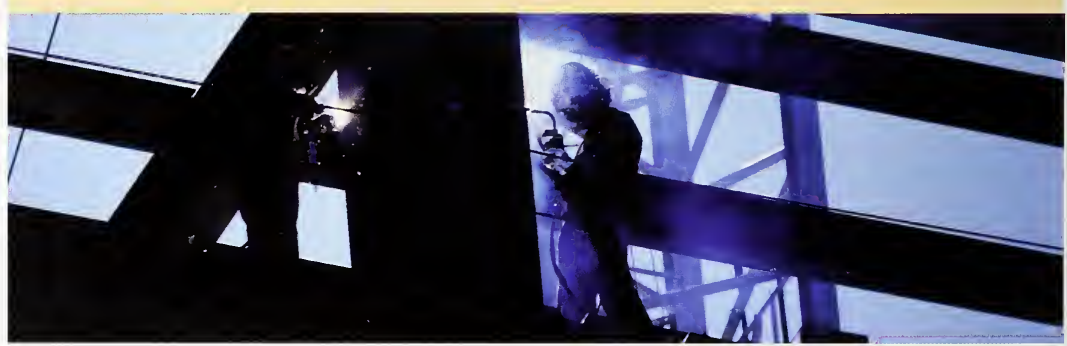




\section{Stainless Steels (chip)}

Unit Size: $150 \mathrm{~g}$ (unless otherwise noted)

$\begin{array}{ll}\text { SRM } & \text { Description } \\ 339 & \text { SAE } 303 \mathrm{Se}(17 \mathrm{Cr}-9 \mathrm{Ni}-0.2 \mathrm{Se}) \\ 101 \mathrm{~g} & \text { AISI } 304 \mathrm{~L}(18 \mathrm{Cr}-10 \mathrm{Ni}) \\ 343 \mathrm{a} & \text { AISI } 431(16 \mathrm{Cr}-2 \mathrm{Ni}) \\ 123 \mathrm{c} & \text { AISI } 348(17 \mathrm{Cr}-11 \mathrm{Ni}-0.6 \mathrm{Nb}) \\ 121 \mathrm{~d} & \text { AISI } 321(17 \mathrm{Cr}-11 \mathrm{Ni}-0.3 \mathrm{Ti}) \\ 160 \mathrm{~b} & \text { AISI } 316(18 \mathrm{Cr}-12 \mathrm{Ni}-2 \mathrm{Mo}) \\ 166 \mathrm{c} & \text { AISI } 316 \mathrm{~L} \mathrm{Low} \mathrm{Carbon} \mathrm{Stainless} \mathrm{Steel}(100 \mathrm{~g}) \\ 893 & \text { SAE } 405(\mathrm{Cr}) \\ 895 & \text { SAE } 201(\mathrm{Cr}-\mathrm{Mn}) \\ 73 \mathrm{C} & \text { SAE } 420(13 \% \mathrm{Cr})\end{array}$

\section{Stainless Steels (disk)}

Unit Size: $32 \mathrm{~mm}$ diameter $\times 19 \mathrm{~mm}$

$\begin{array}{ll}\text { SRM } & \text { Description } \\ 1219 & \text { AISI } 431(16 \mathrm{Cr}-2 \mathrm{Ni}) \\ 1172 & \text { AISI } 348(17 \mathrm{Cr}-11 \mathrm{Ni}-0.6 \mathrm{Nb}) \\ 1223 & \text { Chromium Steel } \\ 1297 & \text { SAE } 201 \\ 1295 & \text { SAE } 405 \\ \text { C1296 } & \text { SAE } 460 \\ \text { C1153a } & \text { (17Cr - 9Ni) } \\ \text { C1152a } & \text { (18Cr - 11Ni) } \\ 1155 & \text { AISI 316 (18Cr - 12Ni - 2Mo }) \\ \text { C1154a } & \text { Stainless Steel, }(19 \mathrm{Cr}-13 \mathrm{Ni}) \\ \text { C1151a } & \text { Stainless Steel, }(23 \mathrm{Cr}-7 \mathrm{Ni}) \\ 1171 & \text { AISI 321 (17 Cr - 11Ni - 0.3Ti) }\end{array}$




\section{Specialty Steels (disk)}

$\begin{array}{lll}\text { SRM } & \text { Description } & \text { Unit Size } \\ 1158 & \text { High Nickel Steel, } 36 \% \text { Nickel } & 32 \mathrm{~mm} \text { diameter } \times 19 \mathrm{~mm} \\ 1772 & \text { S-7 Tool Steel } & 34 \mathrm{~mm} \text { diameter } \times 19 \mathrm{~mm} \\ 1157 & \text { AlSI M2, Tool Steel } & 32 \mathrm{~mm} \text { diameter } \times 19 \mathrm{~mm} \\ 1233 & \text { Valve Steel } & 35 \mathrm{~mm} \text { diameter } \times 19 \mathrm{~mm}\end{array}$

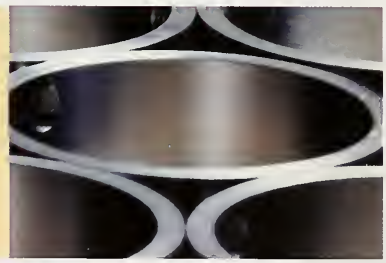

\section{Tool Steels (chip)}

Unit Size: $150 \mathrm{~g}$
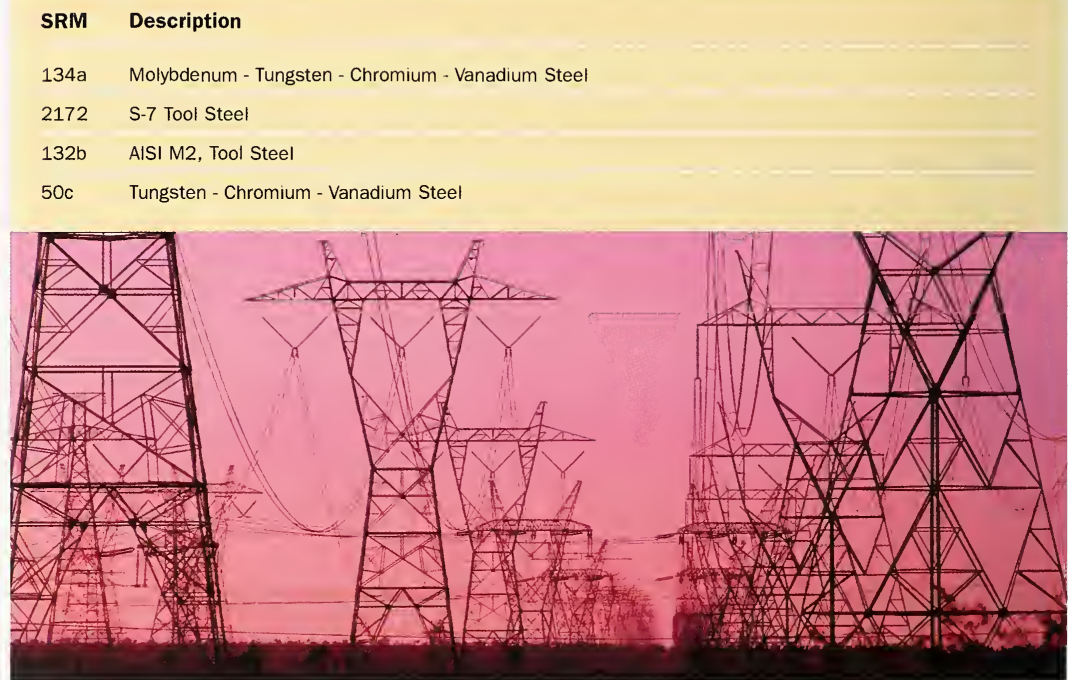
Cast Steels, White Cast Irons, and Ductile Irons (disk)

Unit Size: $32 \mathrm{~mm}$ diameter $\times 19 \mathrm{~mm}$

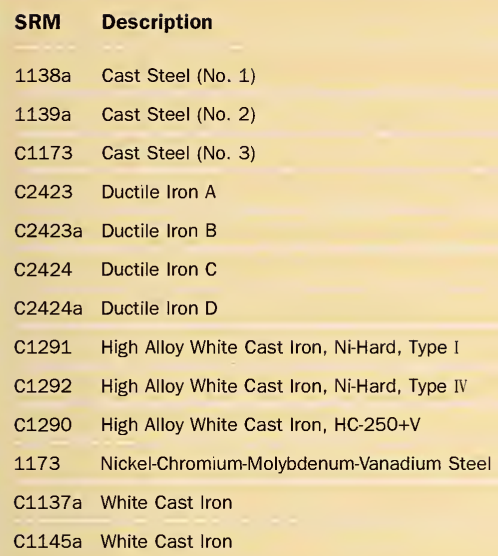

\section{Steelmaking Alloys (fine powder)}

Unit Size: $150 \mathrm{~g}$

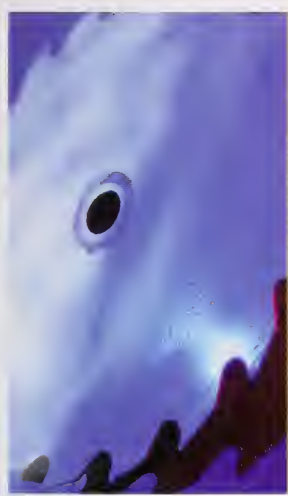

$\begin{array}{ll}\text { SRM } & \text { Description } \\ 57 a & \text { Silicon Metal } \\ 58 a & \text { Ferrosilicon (73\% Silicon-Regular Grade) } \\ 59 a & \text { Ferrosilicon } \\ 64 c & \text { High Carbon Ferrochromium } \\ 68 c & \text { High Carbon Ferromanganese } \\ 90 & \text { Ferrophosphorus } \\ 195 & \text { Ferrosilicon (75 \% Silicon High Purity Grade) } \\ 196 & \text { Low Carbon Ferrochromium } \\ 347 & \text { Magnesium Ferrosilicon } \\ 689 & \text { Silicon Ferrochromium }\end{array}$




\section{Cast Irons (chip)}

Unit Size: $150 \mathrm{~g}$

$\begin{array}{ll}\text { SRM } & \text { Title } \\ 4 \mathrm{~L} & \text { Cast Iron } \\ 5 \mathrm{~m} & \text { Cast Iron } \\ 6 \mathrm{~g} & \text { Cast Iron } \\ 122 \mathrm{i} & \text { Cast Iron } \\ 7 \mathrm{~g} & \text { High Phosphorus Cast Iron } \\ 115 \mathrm{a} & \text { Copper-Nickel-Chromium Cast Iron } \\ 341 & \text { Ductile Cast Iron } \\ 334 & \text { Gray Cast Iron (Carbon and Sulfur) } \\ 890 & \text { High-Alloy White Cast Iron, HC 250+V } \\ 891 & \text { High-Alloy White Cast Iron, Nickel-Hard, Type I } \\ 892 & \text { High-Alloy White Cast Iron, Nickel-Hard, Type IV } \\ 82 \mathrm{~b} & \text { Nickel Chromium Cast Iron } \\ 107 \mathrm{c} & \text { Nickel-Chromium-Molybdenum Cast Iron } \\ 342 \mathrm{a} & \text { Nodular Cast Iron } \\ 338 & \text { White Cast Iron, Carbon and Sulfur }\end{array}$

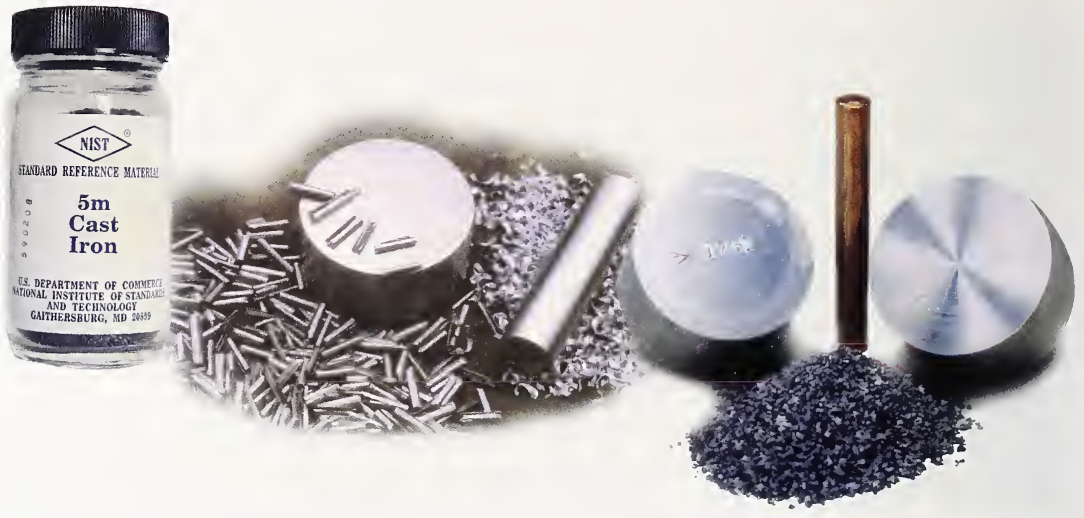




$\begin{array}{ll}\text { SRM } & \text { Description } \\ 866 & \text { Incoloy }{ }^{\mathrm{TM}} 800 \\ 867 & \text { Incoloy }^{\mathrm{TM}} 825 \\ 1230 & \text { High Temperature Alloy A286 } \\ 1246 & \text { Incoloy } 800 \\ 1247 & \text { Incoloy }{ }^{\mathrm{TM}} 825 \\ 1250 & \text { High Temperature Alloy (Fe - Ni - Co) } \\ \text { C2400 } & \text { High Alloy Steel, ACl 17/4 PH } \\ \text { C2401 } & \text { High Alloy Steel ACl-CD-4M Cu }\end{array}$

\section{Unit Size}

$100 \mathrm{~g}$

$100 \mathrm{~g}$

disk: $32 \mathrm{~mm}$ diameter $\times 19 \mathrm{~mm}$

disk: $35 \mathrm{~mm}$ diameter $\times 19 \mathrm{~mm}$

disk: $35 \mathrm{~mm}$ diameter $\times 19 \mathrm{~mm}$

disk: $32 \mathrm{~mm}$ diameter $\times 19 \mathrm{~mm}$

disk: $32 \mathrm{~mm}$ diameter $\times 19 \mathrm{~mm}$

disk: $32 \mathrm{~mm}$ diameter $\times 19 \mathrm{~mm}$

\section{Gases in Metals: Iron and Steel (rod)}

These SRMs are certified for oxygen content. Materials certified for nitrogen are noted.

$\begin{array}{lll}\text { SRM } & \text { Description } & \text { Rod Size (mm) } \\ 1089 * & \begin{array}{l}\text { Gasometric Standard, set includes: } \\ \\ \text { SRM 1095 AISI 4340 Steel }\end{array} & \\ & \text { SRM 1096 AISI 94B17 Steel, Modified** } & 6.4 \times 102 \\ & \text { SRM 1097 Cr-V Steel, Modified } & 6.4 \times 102 \\ & \text { SRM 1098 High Carbon Steel** } & 6.4 \times 102 \\ & \text { SRM 1099 Electrolytic Iron } & 6.4 \times 102 \\ 1754 & \text { AISI 4320 Oxygen in Low Alloy Steel,** } & 6.4 \times 102 \\ 1090 & \text { Oxygen in Ingot Iron } & 9.5 \times 9.5 \times 102 \\ 1094 & \text { Oxygen in Maraging Steel } & 6.35 \times 102 \\ 1091 \text { a } & \text { AISI 431 Oxygen in Stainless Steel } & 0.6 \times 82 \\ 1093 & \text { Oxygen in Valve Steel } & 7.9 \times 102\end{array}$

* These SRMs are sold only as a set designated SRM 1089.

** In addition to being certified for oxygen, these SRMs are also certified for nitrogen. 


\section{NONFERROUS METALS \\ Aluminum Base Alloys (chip and disk)}

SRMs 1710 through 1715 are specially prepared to include low levels of cadmium and lead encountered in the analysis of recycled aluminum.

$\begin{array}{ll}\text { SRM } & \text { Description } \\ 87 a & \text { Silicon - Aluminum Alloy } \\ 855 a & \text { Aluminum Casting Alloy } 356 \\ 856 a & \text { Aluminum Casting Alloy } 380, \text { Fine Millings } \\ 858 & \text { Alloy } 6011, \text { Modified } \\ 1258 & \text { Alloy } 6011, \text { Modified } \\ 859 & \text { Alloy } 7075 \\ 1259 & \text { Alloy } 7075 \\ 1710 & \text { Alloy } 3004 \\ 1711 & \text { Alloy } 3004 \\ 1712 & \text { Alloy } 3004 \\ 1713 & \text { Alloy } 5182 \\ 1714 & \text { Alloy } 5182 \\ 1715 & \text { Alloy } 5182\end{array}$

Unit Size

$75 \mathrm{~g}$

$30 \mathrm{~g}$

$30 \mathrm{~g}$

$35 \mathrm{~g}$

disk: $35 \mathrm{~mm}$ diameter $\times 19 \mathrm{~mm}$

$35 \mathrm{~g}$

disk: $35 \mathrm{~mm}$ diameter $\times 19 \mathrm{~mm}$ disk: $63 \mathrm{~mm}$ diameter $\times 19 \mathrm{~mm}$ disk: $63 \mathrm{~mm}$ diameter $\times 19 \mathrm{~mm}$ disk: $63 \mathrm{~mm}$ diameter $\times 19 \mathrm{~mm}$ disk: $63 \mathrm{~mm}$ diameter $\times 19 \mathrm{~mm}$ disk: $63 \mathrm{~mm}$ diameter $\times 19 \mathrm{~mm}$ disk: $63 \mathrm{~mm}$ diameter $\times 19 \mathrm{~mm}$

\section{Cobalt Base Alloys (chip and disk)}

$\begin{array}{ll}\text { SRM } & \text { Description } \\ 862 & \text { High Temperature Alloy L605 } \\ 1242 & \text { High Temperature Alloy L605 } \\ 1775 & \text { Refractory Alloy MP-35-N } \\ 2175 & \text { Refractory Alloy MP-35-N }\end{array}$

Unit Size

chip: $100 \mathrm{~g}$

disk: $35 \mathrm{~mm}$ diameter $\times 19 \mathrm{~mm}$

disk: $35 \mathrm{~mm}$ diameter $\times 19 \mathrm{~mm}$

chip: $50 \mathrm{~g}$ 


\section{Copper "Benchmark" (chip and rod)}

Unit Size: Chip: $50 \mathrm{~g}$

Rod: $6.4 \mathrm{~mm} \times 103 \mathrm{~mm}$

\begin{tabular}{lr}
\multicolumn{2}{c}{ SRM } \\
\hline Chip & Rod \\
395 & 495 \\
396 & 496 \\
& 457 \\
398 & 498 \\
399 & 499 \\
400 & 500 \\
454 &
\end{tabular}
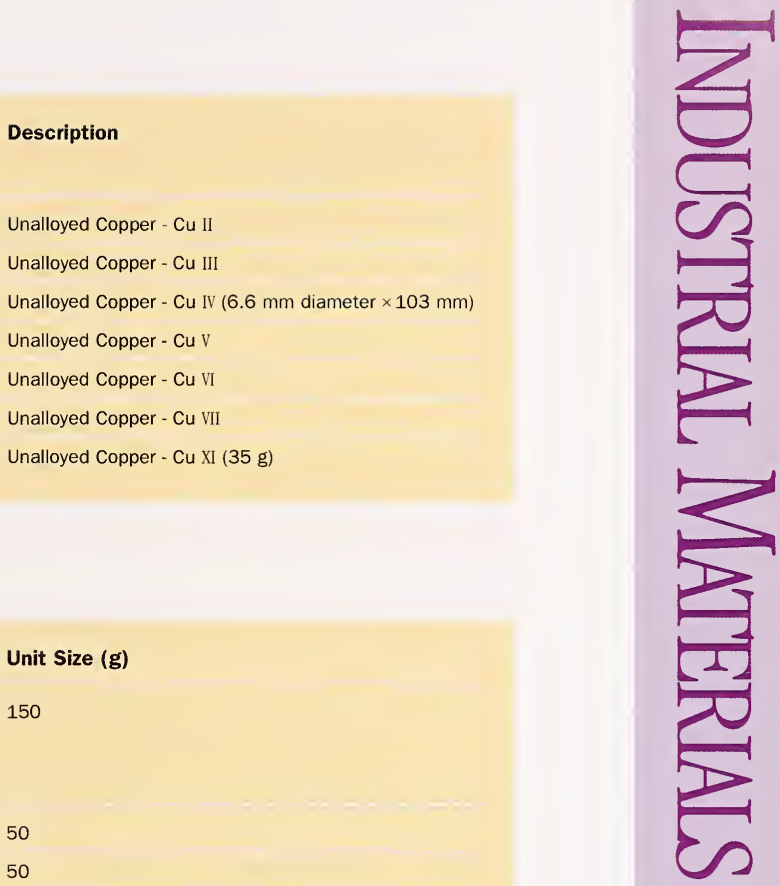

\section{Copper Base Alloys (chip and rod)}

$\begin{array}{ll}\text { SRM } & \text { Description } \\ 158 a & \text { Silicon, Bronze }\end{array}$

Beryllium-Copper

$\begin{array}{ll}458 & 17510 \\ 459 & 17200 \\ 460 & 17300\end{array}$

\section{Phosphor-Bronze}

871

CDA 521

872

CDA 544

\section{Cupro-Nickel}

$\begin{array}{ll}874 & 10 \% \text { CDA 706, High-Purity } \\ 875 & 10 \% \text { CDA 706, Doped }\end{array}$

Nickel-Silver

$\begin{array}{ll}879 & \text { CDA } 762 \\ 880 & \text { CDA } 770 \\ 1034 & \text { Unalloyed Copper } \\ 1035 & \text { Leaded-Tin Bronze Alloy }\end{array}$

\section{Unit Size (g)}

150

50

50

50

100

100

100

100

100

100

rod: $6.35 \mathrm{~mm}$ diameter $\times 103 \mathrm{~mm}$

50

(continued) 


\section{Copper Base Alloys (block and disk)}

The 1100 series SRMs are wrought disks $32 \mathrm{~mm}$ diameter $\times 19 \mathrm{~mm}$. The $\mathrm{C} 1100$ series SRMs are chill cast blocks $32 \mathrm{~mm}$ square $\times 19 \mathrm{~mm}$. Both forms have nearly identical elemental compositions.

\begin{tabular}{|c|c|c|}
\hline \multicolumn{2}{|c|}{ SRM } & \multirow[t]{2}{*}{ Description } \\
\hline Disk & Block & \\
\hline 1104 & & Free-Cutting Brass \\
\hline 1107 & & Naval Brass B \\
\hline 1108 & & Naval Brass C \\
\hline 1110 & & Red Brass B \\
\hline 1111 & & Red Brass C \\
\hline 1112 & $\mathrm{C} 1112$ & Gilding Metal A \\
\hline 1113 & C1113 & Gilding Metal B \\
\hline 1114 & C1114 & Gilding Metal C \\
\hline 1115 & C1115 & Commercial Bronze A \\
\hline 1116 & C1116 & Commercial Bronze B \\
\hline \multirow[t]{2}{*}{1117} & $\mathrm{C} 1117$ & Commercial Bronze C \\
\hline & $\mathrm{C} 1122$ & Beryllium-Copper \\
\hline $1276 a$ & & CDA 715 Cupro-Nicke \\
\hline
\end{tabular}

\section{Lead Base Alloys (disk and powder forms)}

\begin{tabular}{ll}
\multicolumn{2}{c}{ SRM } \\
\hline Powder & Disk \\
1129 & \\
$127 \mathrm{~b}$ & 1131 \\
$53 \mathrm{e}$ & 1132
\end{tabular}

\author{
Description \\ Solder $63 \mathrm{Sn}-37 \mathrm{~Pb}$ \\ Solder $40 \mathrm{Sn}-60 \mathrm{~Pb}$ \\ Lead Base Bearing Metal \\ $(84 \mathrm{~Pb}-10 \mathrm{Sb}-6 \mathrm{Sn})$
}

$\begin{array}{ll}\frac{\text { Unit Size }(\mathbf{g})}{\text { Powder }} & \text { Disk } \\ 200 & \\ 150 & 32 \mathrm{~mm} \text { diameter } \times 19 \mathrm{~mm} \\ 150 & 32 \mathrm{~mm} \text { diameter } \times 19 \mathrm{~mm}\end{array}$

\section{Lead Base Materials (disk)}

Unit Size: $50 \mathrm{~mm}$ diameter $\times 16 \mathrm{~mm}$

$\begin{array}{ll}\text { SRM } & \text { Description } \\ \text { C2415 } & \text { Battery Lead } \\ \text { C2416 } & \text { Bullet Lead } \\ \text { C2417 } & \text { Lead Base Alloy } \\ \text { C2418 } & \text { High Purity Lead }\end{array}$




\section{Nickel Oxides (powder)}

Unit Size: $25 \mathrm{~g}$

$\begin{array}{ll}\text { SRM } & \text { Description } \\ 671 & \text { Nickel Oxide No. } 1 \\ 672 & \text { Nickel Oxide No. } 2 \\ 673 & \text { Nickel Oxide No. } 3\end{array}$

Nickel Base Alloys (chip and disk)

\begin{tabular}{|c|c|}
\hline SRM & Description \\
\hline $349 a$ & Waspaloy ${ }^{\top M}$ \\
\hline 864 & Inconel ${ }^{\mathrm{MM}} 600$ \\
\hline 865 & Inconel ${ }^{\mathrm{MM}} 625$ \\
\hline 882 & Nickel-Copper Alloy (65Ni - 31Cu - 3Al) \\
\hline 1159 & Electronic and Magnetic Alloy Ni-Fe \\
\hline 1160 & Electronic and Magnetic Alloy Ni-Mo \\
\hline 1243 & Waspaloy ${ }^{\top M}$ \\
\hline 1244 & Inconel ${ }^{\top M} 600$ \\
\hline C1248 & Nickel-Copper Alloy (66Ni - 30Cu) \\
\hline 1249 & Inconel ${ }^{\mathrm{MM}} 718$ \\
\hline 2402 & Hastelloy ${ }^{\top M} \mathrm{C}$ \\
\hline
\end{tabular}

Unit Size
$150 \mathrm{~g}$
$100 \mathrm{~g}$
$100 \mathrm{~g}$
$100 \mathrm{~g}$
disk: $31 \mathrm{~mm}$ diameter $\times 19 \mathrm{~mm}$
disk: $31 \mathrm{~mm}$ diameter $\times 19 \mathrm{~mm}$
disk: $34 \mathrm{~mm}$ diameter $\times 19 \mathrm{~mm}$
disk: $35 \mathrm{~mm}$ diameter $\times 19 \mathrm{~mm}$
disk: $32 \mathrm{~mm}$ diameter $\times 19 \mathrm{~mm}$
disk: $41 \mathrm{~mm}$ diameter $\times 19 \mathrm{~mm}$
disk: $32 \mathrm{~mm}$ diameter $\times 19 \mathrm{~mm}$

Trace Elements in Nickel Base Superalloys (chip)

Unit Size: $35 \mathrm{~g}$

$\begin{array}{ll}\text { SRM } & \text { Description } \\ 897 & \text { "Tracealloy" A } \\ 898 & \text { "Tracealloy" B } \\ 899 & \text { "Tracealloy" C }\end{array}$

\section{Elemental Composition}

$\mathrm{Pb}, \mathrm{Sc}, \mathrm{Te}, \mathrm{TI}$ 
Tin Base Alloys (chip)

SRM Description

54d Tin Base Bearing Metal

Titanium Base Alloys (chip and disk)
Unit Size

$170 \mathrm{~g}$

$\begin{array}{ll}\text { SRM } & \text { Description } \\ 641 & 8 \mathrm{Mn}(\mathrm{A}) \\ 642 & 8 \mathrm{Mn}(\mathrm{B}) \\ 643 & 8 \mathrm{Mn}(\mathrm{C}) \\ 647 & 6 \mathrm{Al}-2 \mathrm{Mo}-2 \mathrm{Sn}-4 \mathrm{Zr} \\ 648 & 5 \mathrm{Al}-2 \mathrm{Sn}-2 \mathrm{Cr}-4 \mathrm{Mo} \\ 649 & 15 \mathrm{~V}-3 \mathrm{Al}-2 \mathrm{Cr}-3 \mathrm{Sn} \\ 650 & \text { Unalloyed Titanium A } \\ 651 & \text { Unalloyed Titanium B } \\ 654 \mathrm{~b} & 6 \mathrm{Al}-4 \mathrm{~V} \\ 1128 & 15 \mathrm{~V}-3 \mathrm{Al}-3 \mathrm{Cr}-3 \mathrm{Sn} \\ 2431 & 6 \mathrm{Al}-2 \mathrm{Sn}-4 \mathrm{Zr}-6 \mathrm{Mo} \\ 2432 & 10 \mathrm{~V}-2 \mathrm{Fe}-3 \mathrm{Al} \\ 2433 & 8 \mathrm{Al}-1 \mathrm{Mo}-1 \mathrm{~V}\end{array}$

Hydrogen in Titanium (platelet)

SRM

Description

352c Hydrogen in Unalloyed Titanium

\section{Unit Size (g)}

disk: $32 \mathrm{~mm}$ diameter $\times 19 \mathrm{~mm}$

disk: $32 \mathrm{~mm}$ diameter $\times 19 \mathrm{~mm}$

disk: $32 \mathrm{~mm}$ diameter $\times 19 \mathrm{~mm}$

50

50

50

30

30

disk: $31 \mathrm{~mm}$ diameter $\times 19 \mathrm{~mm}$ disk: $35 \mathrm{~mm}$ diameter $\times 19 \mathrm{~mm}$ 50

50

50

\section{Zirconium Base Alloys (chip)}

SRM

Description

$360 \mathrm{~b}$
Unit Size

$20 \mathrm{~g}$

$100 \mathrm{~g}$ 
Zinc Base Alloys (chip and disk)

$\begin{array}{ll}\text { SRM } & \text { Description } \\ 94 \mathrm{C} & \text { Die Casting Alloy } \\ 625 & \text { ASTM AG 40A Die Casting Alloy } \\ 626 & \text { ASTM AG 40A Die Casting Alloy } \\ 627 & \text { ASTM AG 40A Die Casting Alloy } \\ 628 & \text { ASTM AC 41A Die Casting Alloy } \\ 629 & \text { ASTM AC 41A Die Casting Alloy } \\ 630 & \text { ASTM AC 41A Die Casting Alloy } \\ 631 & \text { Zinc spelter, Modified } \\ 1736 & \text { Zinc-Aluminum (.31 \% Al) Die Casting Alloy } \\ 1737 & \text { Zinc-Aluminum (.63 \% Al) Die Casting Alloy } \\ 1738 & \text { Zinc-Aluminum (.10 \% Al) Die Casting Alloy } \\ 1739 & \text { Zinc-Aluminum (.21 \% Al) Die Casting Alloy } \\ 1740 & \text { Zinc-Aluminum (.42\% Al) Die Casting Alloy } \\ 1741 & \text { Zinc-Aluminum (.52\% Al) Die Casting Alloy } \\ 1742 & \text { Zinc-Aluminum (.79\% Al) Die Casting Alloy } \\ 2139 & \text { Zinc-Aluminum (.80 \% Al) Die Casting Alloy }\end{array}$

\section{CERAMICSAND GLASSES Carbides (powder)}

$\begin{array}{ll}\text { SRM } & \text { Description } \\ 112 b & \text { Silicon Carbide } \\ 276 b & \text { Tungsten Carbide }\end{array}$

\section{Cemented Tungsten Carbides (powder)}

Unit Size: $100 \mathrm{~g}$

\section{Unit Size}

chip: $150 \mathrm{~g}$

disk: $44 \mathrm{~mm}$ diameter $\times 19 \mathrm{~mm}$ disk: $44 \mathrm{~mm}$ diameter $\times 19 \mathrm{~mm}$ disk: $44 \mathrm{~mm}$ diameter $\times 19 \mathrm{~mm}$ disk: $44 \mathrm{~mm}$ diameter $\times 19 \mathrm{~mm}$ disk: $44 \mathrm{~mm}$ diameter $\times 19 \mathrm{~mm}$ disk: $44 \mathrm{~mm}$ diameter $\times 19 \mathrm{~mm}$ disk: $45 \mathrm{~mm}$ diameter $\times 19 \mathrm{~mm}$ disk: $50.8 \mathrm{~mm}$ diameter $\times 12.7 \mathrm{~mm}$ disk: $50.8 \mathrm{~mm}$ diameter $\times 12.7 \mathrm{~mm}$ disk: $50.8 \mathrm{~mm}$ diameter $\times 12.7 \mathrm{~mm}$ disk: $50.8 \mathrm{~mm}$ diameter $\times 12.7 \mathrm{~mm}$ disk: $50.8 \mathrm{~mm}$ diameter $\times 12.7 \mathrm{~mm}$ disk: $50.8 \mathrm{~mm}$ diameter $\times 12.7 \mathrm{~mm}$ disk: $50.8 \mathrm{~mm}$ diameter $\times 12.7 \mathrm{~mm}$ chip: $100 \mathrm{~g}$

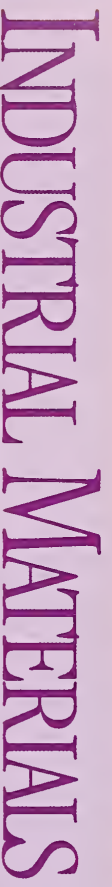

Unit Size (g)

80

75 


\section{Glasses (powder and solid)}

$\begin{array}{lll}\text { SRM } & \text { Description } & \text { Unit Size }(\mathbf{g}) \\ 81 a & \text { Glass Sand } & 75 \\ 89 & \text { Lead-Barium } & 45 \\ 92 & \text { Low-Boron Soda-Lime Powder } & 45 \\ 93 a & \text { High-Boron Borosilicate } & \text { wafer: } 32 \mathrm{~mm} \text { diameter } \times 6 \mathrm{~mm} \\ 165 a & \text { Glass Sand (low Iron) } & 75 \\ 620 & \text { Soda-Lime, Flat } & 3 \text { platelets: } 35 \mathrm{~mm} \times 35 \mathrm{~mm} \times 3 \mathrm{~mm} \\ 621 & \text { Soda-Lime, Container } & 3 \text { disks: } 38 \mathrm{~mm} \text { diameter } \times 5 \mathrm{~mm} \\ 1411 & \text { Soft Borosilicate } & 10 \text { platelets: } 32 \mathrm{~mm} \times 32 \mathrm{~mm} \times 3 \mathrm{~mm} \\ 1412 & \text { Multicomponent } & 8 \text { platelets: } 32 \mathrm{~mm} \times 32 \mathrm{~mm} \times 3 \mathrm{~mm} \\ 1413 & \text { Glass Sand (high alumina) } & 75 \\ 1830 & \text { Soda-Lime, Float } & 3 \text { platelets: } 32 \mathrm{~mm} \times 32 \mathrm{~mm} \times 6 \mathrm{~mm} \\ 1831 & \text { Soda-Lime, Sheet } & 3 \text { platelets: } 37 \mathrm{~mm} \times 37 \mathrm{~mm} \times 3 \mathrm{~mm} \\ 1834 & \text { Fused Ore Glass } & \text { disk: } 30 \mathrm{~mm} \mathrm{diameter} \times 3 \mathrm{~mm}\end{array}$

\section{Trace Elements (powder and wafer)}

These SRMs are for calibrating instruments and evaluating analytical techniques used to determine trace elements in inorganic matrices. SRMs 610 through 617 come in units of 6 wafers with wafer thicknesses of $3 \mathrm{~mm}$ for even numbered SRMs and $1 \mathrm{~mm}$ for odd numbered SRMs.

Also certified for isotopic ratio: ${ }^{87} \mathrm{Sr} /{ }^{86} \mathrm{Sr}=1.20039$

\section{SRM Description \\ 607 Trace Elements in Potassium Feldspar (5 g)}

\section{Trace Elements in Glass}

$610 / 611$

$612 / 613$

$614 / 615$

$616 / 617$

\section{Certified Elements}
33 elements
33 elements
33 elements
33 elements 
C E M EN T S

Portland Cements (powder)

SRM

Unit Size

Calcium Aluminate Cement

$\begin{array}{ll}1882 a & 4 \times 5 g \\ 1883 a & 4 \times 5 g\end{array}$

Portland Cement

$1880 a$

$4 \times 5 g$

$1881 a$

$4 \times 5 g$

$1884 a$

$4 \times 5 g$

$1885 a$

$4 \times 5 g$

1886 (Cranberry cap)

$3 \times 5 g$

1886a (In Prep)

$4 \times 5 \mathrm{~g}$

1887 (Brown cap)

$3 \times 5 g$

$1887 a$

$4 \times 5 \mathrm{~g}$

1888a (In Prep)

$4 \times 5 g$

1889a

$4 \times 5 g$

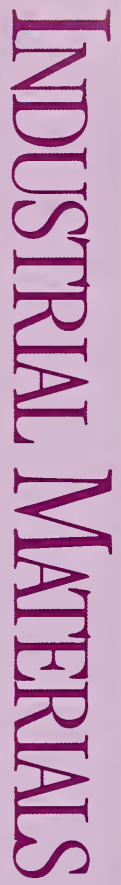

\section{Portland Cement Clinkers (solid)}

Unit Size: $3 \times 10 \mathrm{~g}$

SRM

Portland Cement Clinkers (5 phases certified)

2686

2687

2688 


\section{PHYSICAL PROPERTIES}

65 Ion Activity

68 Polymeric Properties

70 Thermodynamic Properties

74 Optical Properties

77 Electrical Properties

78 Optoelectronics

78 Metrology

81 Ceramics and Glasses

83 X-ray Spectrometry 


\section{ION ACTIVITY \\ pH Calibration}

\begin{tabular}{|c|c|c|c|}
\hline SRM & Description & $\begin{array}{l}\mathrm{pH}(\mathrm{S}) \text { Values } \\
\left(\text { at } 25^{\circ} \mathrm{C}\right)\end{array}$ & Unit Size (g) \\
\hline 2193 & Calcium Carbonate (In Prep) & - & - \\
\hline $723 d$ & Tris(Hydroxymethyl) aminomethane (In Prep) & - & - \\
\hline $185 \mathrm{~g}$ & Potassium Hydrogen Phthalate & 4.006 & 60 \\
\hline 188 & Potassium Hydrogen Tartrate & 3.557 & 60 \\
\hline $189 b$ & Potassium Tetroxalate & 1.719 & 65 \\
\hline $187 d$ & Sodium Tetraborate Decahydrate (Borax) & 9.182 & 30 \\
\hline \multicolumn{4}{|c|}{$\begin{array}{l}\text { Admixtures } \\
\text { Unit Size: } 3\end{array}$} \\
\hline $\begin{array}{l}186 \text { If } \\
186 \text { IIf }\end{array}$ & Potassium Dihydrogen Phosphate & $6.860^{*}$ & \\
\hline 186Iff & Disodium Hydrogen Phosphate & $7.414 * *$ & \\
\hline $\begin{array}{l}191 b \\
192 b\end{array}$ & $\begin{array}{l}\text { Sodium Bicarbonate }(25 \mathrm{~g}) \\
\text { Sodium Carbonate }\end{array}$ & $10.015 *$ & \\
\hline
\end{tabular}

*This $\mathrm{pH}$ results only when the two SRMs listed are used as an admixture in solution.

** Physiological buffer preparation. 


\section{Biological Buffer Systems}

Unit Size: $60 \mathrm{~g}$

\begin{tabular}{llll} 
SRM & Description & \multicolumn{1}{c}{$\mathbf{p H}(\mathbf{S})$ Values (at $\mathbf{3 7} \mathbf{\circ}^{\circ} \mathbf{C}$ ) } \\
\cline { 3 - 4 } & & $\mathbf{0 . 0 5}$ molal & $\mathbf{0 . 0 8}$ molal \\
2181 & HEPES Free Acid & $7.364^{*}$ & $7.373^{*}$ \\
2182 & NaHEPESate & & \\
2183 & MOPSO Free Acid & $6.699^{*}$ & $6.694^{*}$ \\
2184 & NaMOPSOate & &
\end{tabular}

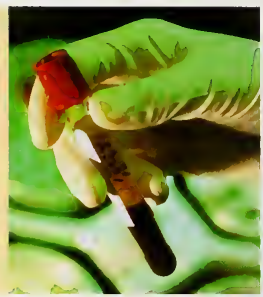

*This $\mathrm{pH}$ results only when the two SRMs listed are used as an admixture in solution.

\section{pD Calibration}

\begin{tabular}{|c|c|c|c|}
\hline SRM & Description & $\begin{array}{l}\mathrm{pD}(\mathrm{S}) \text { Values } \\
\left(\text { at } 25^{\circ} \mathrm{C}\right)\end{array}$ & Unit Size (g) \\
\hline 2185 & Potassium Hydrogen Phthalate & 4.518 & 60 \\
\hline $\begin{array}{l}2186 I \\
2186 I I\end{array}$ & $\begin{array}{l}\text { Potassium Dihydrogen Phosphate } \\
\text { Disodium Hydrogen Phosphate }\end{array}$ & $7.428 *$ & $\begin{array}{l}30 \\
30\end{array}$ \\
\hline $\begin{array}{l}2191 a \\
2192 a\end{array}$ & $\begin{array}{l}\text { Sodium Bicarbonate } \\
\text { Sodium Carbonate }\end{array}$ & $10.732^{*}$ & $\begin{array}{l}30 \\
30\end{array}$ \\
\hline
\end{tabular}

*This $p D$ results only when the two SRMs listed are used as an admixture in solution.

\section{Ion-Selective Electrode Calibration}

$\begin{array}{ll}\text { SRM } & \text { Description } \\ 2201 & \text { Sodium Chloride } \\ 2202 & \text { Potassium Chloride } \\ 2203 & \text { Potassium Fluoride }\end{array}$

Certified Property

$\mathrm{pNa}, \mathrm{pCl}$

$\mathrm{pK}, \mathrm{pCl}$

$\mathrm{pF}$
Unit Size (g)

125

160

125 


\section{Electrolytic Conductivity}

SRM Description

$3190 \mathrm{HCl}$ in Deionized Water (In Prep)

$\mathrm{KCl}$ in Deionized Water

3191 (In Prep)

3192 (In Prep)

3193

3194

3195

KCl in n-Propanol/Deionized Water

3198 (In Prep)

3199

$\mathrm{NaCl}$ in deionized Water

3196 (In Prep)

\section{Positive Electrophoretic Mobility}

$\begin{array}{ll}\text { SRM } & \text { Description } \\ 1980 & \text { Goethite }(\alpha-\mathrm{FeOOH})\end{array}$

Certified Property

$+\mu \mathrm{E}, 2.53 \mu \mathrm{m} \cdot \mathrm{cm} / \mathrm{V} \cdot \mathrm{s}$
Nominal Conductivity $(\mu \mathrm{S} / \mathrm{cm})$

一

1000

10000

100000

15 


\section{POLYMERIC PROPERTIES}

\section{Molar Mass/Molecular Weight $\left(\mathbf{M}_{\mathrm{w}}\right)$}

SRM $\quad M_{w}(g / m o l)$

Unit Size (g)

Poly(ethylene oxide)

$1924 M_{w} \approx 120900\left(M_{w} / M_{n} \approx 1.04\right)$

0.2

$1923 \quad M_{w} \approx 26900\left(M_{w} / M_{n} \approx 1.06\right)$

Poly(methylmethacrylate)

1489* $M_{n} \approx 115000\left(M_{w} / M_{n} \leq 1.1\right)$

1.1

1488* $M_{n} \approx 29300\left(M_{w} / M_{n} \leq 1.1\right)$

2

$1487^{*} \quad M_{w} \approx 6300$

Polyethylene

$2887^{*} \quad M_{w} \approx 196400$

0.3

2885* $M_{w} \approx 6280$

0.3

2886* $\quad M_{w} \approx 87000$

Polyethylene, linear

1475a* $M_{w} \approx 52000\left(M_{w} / M_{n} \approx 2.90\right)$ (see also melt flow)

50

1484a* $M_{w} \approx 119600\left(M_{w} / M_{n} \approx 1.19\right)$

0.3

1482a* $M_{w} \approx 13600\left(M_{w} / M_{n} \approx 1.19\right)$

0.4

1483* $M_{w} \approx 32100\left(M_{w} / M_{n} \approx 1.11\right)$

Polystyrene, linear, broad molecular weight distribution

$706 \mathrm{a} \quad \mathrm{M}_{\mathrm{w}} \approx 285000$

Polystyrene, linear, narrow molecular weight distribution
1478* $M_{w} \approx 37400\left(M_{w} / M_{n} \approx 1.04\right)$
705a* $\quad M_{w} \approx 179300\left(M_{w} / M_{n} \approx 1.07\right)$
$1479 \quad M_{w} \approx 1050000$
Polyurethane
$1480 \quad M_{w} \approx 47300$

2

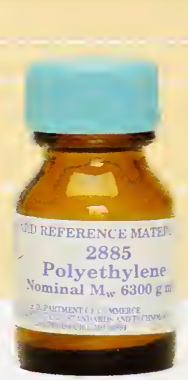

* Also certified for viscosity 
Melt Flow Rate

$\begin{array}{llll}\text { SRM } & \text { Description } & \begin{array}{l}\text { Melt Flow Rate } \\ \text { (g/10 min) }\end{array} & \text { Unit Size (g) } \\ & & 1.17 & 60 \\ 1473 a & \text { Polyethylene Resin, Low Density } & 2.02 & 50 \\ 1475 a & \text { Polyethylene, Linear } & 5.03 & 60 \\ 1474 & \text { Polyethylene Resin } & 0.186 & 9080 \\ 1497 & \text { Polyethylene Gas Pipe Resin, Pigmented } & 0.26 & 908 \\ 1496 & \text { Polyethylene Gas Pipe Resin, Unpigmented } & \end{array}$

\section{Viscosity}

SRM Description

Unit Size $(\mathrm{mL})$

2490 Non-Newtonian Polymer Solution for Rheology (Polyisobutylene Dissolved in 2,6,10,14-Tetramethylpentadecane)

100

\section{Biomaterials}

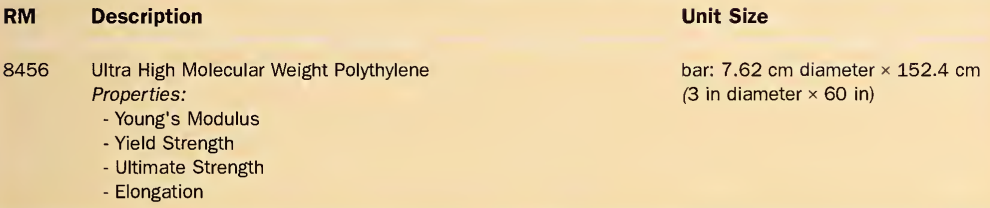

Unit Size

bar: $7.62 \mathrm{~cm}$ diameter $\times 152.4 \mathrm{~cm}$

( 3 in diameter $\times 60$ in) 


\section{THERMODYNAMIC PROPERTIES \\ Calorimetry - Combustion}

\begin{tabular}{|c|c|}
\hline SRM & Description \\
\hline 39j & Benzoic Acid \\
\hline $2692 b$ & Coal, Bituminous: $\% \mathrm{~S}=1.170$ \\
\hline $2685 b$ & Coal, Bituminous: $\% S=4.730$ \\
\hline $2682 b$ & Coal, Sub-Bituminous: $\% \mathrm{~S}=0.4917$ \\
\hline 2151 & Nicotinic Acid \\
\hline $2684 b$ & $\begin{array}{l}\text { Coal, Bituminous, Sulfur and Mercury: } \% \mathrm{~S}=3.08 \\
\mathrm{Hg}=97.4 \mu \mathrm{g} / \mathrm{kg}\end{array}$ \\
\hline 1657 & Synthetic Refuse-Derived Fuel \\
\hline $2683 b$ & $\begin{array}{l}\text { Sulfur and Mercury in Coal: \% S = 1.955, } \\
\mathrm{Hg}=90.0 \mu \mathrm{g} / \mathrm{kg}\end{array}$ \\
\hline 1656 & Thianthrene \\
\hline 2152 & Urea \\
\hline
\end{tabular}

\begin{tabular}{ll}
$\begin{array}{l}\text { Heat of Combustion } \\
(\mathbf{M J} / \mathbf{k g})^{*}\end{array}$ & $\begin{array}{l}\text { Unit Size } \\
(\mathbf{g})\end{array}$ \\
26.434 & 30 \\
$(32.81)^{* *}$ & 50 \\
$(26.94)^{* *}$ & 50 \\
$(25.66)^{* *}$ & 50 \\
22.184 & 25 \\
$28.56 * *$ & 50 \\
\hline $13.87^{* *}$ & 100 \\
30.62 & 50 \\
\hline 33.480 & 30 \\
\hline 10.536 & 25 \\
\hline
\end{tabular}

* The calorific values (MJ/kg) may decrease upon the aging or normal oxidation of the coals. NIST will continue to monitor these calorific values and report any substantive change to the purchaser.

** Gross calorific value or HHV (Higher Heating Value).

\section{Calorimetry - Solution}

$\begin{array}{llll}\text { SRM } & \text { Description } & \text { Heat of Solution } & \text { Unit Size } \\ 1655 & \begin{array}{l}\text { Potassium Chloride } \\ \text { (Water Solution Calorimetry) }\end{array} & \text { Absorbed }(235.86 \mathrm{~J} / \mathrm{g}) & 30 \mathrm{~g}\end{array}$

\section{Enthalpy and Heat Capacity}

$\begin{array}{llll}\text { SRM } & \text { Description } & \text { Unit Size } & \text { Temperature Range (K) } \\ \text { RM 5 } & \text { Copper } & 1.9 \mathrm{~cm} \text { diameter } 12 \mathrm{~cm} & 25 \text { to } 300 \\ 781 \mathrm{D} 2 & \text { Molybdenum } & 0.64 \mathrm{~cm} \text { diameter } 10 \mathrm{~cm} & 273.15 \text { to } 2800 \\ 705 a & \begin{array}{l}\text { Polystyrene } \\ \text { (Molecular Weight: } 170900 \mathrm{~g} / \mathrm{mol})\end{array} & 5 \mathrm{~g} & 10 \text { to } 350 \\ 720 & \text { Synthetic Sapphire } & 15 \mathrm{~g} & 10 \text { to } 2250\end{array}$




\section{Differential Scanning Calorimetry}

$\begin{array}{lllll}\text { SRM } & \text { Description } & \begin{array}{l}\text { Melting } \\ \text { Temperature (K) }\end{array} & \begin{array}{l}\text { Enthalpy of } \\ \text { Fusion }(\mathbf{J} / \mathrm{g})\end{array} & \text { Unit Size } \\ 2222 & \text { Biphenyl }(99.984 \%) & 342.41 & 120.41 & 1 \mathrm{~g} \\ 2232 & \text { Indium }(99.9999 \%) & 156.5985{ }^{\circ} \mathrm{C} & 28.51 & 1 \mathrm{~g} \\ 2225 & \text { Mercury } & 234.30 & 11.469 & 2.5 \mathrm{~g} \\ 2220 & \text { Tin (99.9995\%) } & 505.10 & 60.2 & (2.5 \times 2.5 \times 0.0127) \mathrm{cm} \\ 1514 & \text { Thermal Analysis } & 4 \text { levels of p-ABA }(0.0 \mathrm{~mol} \% \text { to } 5.0 \mathrm{~mol} \%) & 4 \times 0.5 \mathrm{~g}\end{array}$

\section{Differential Thermal Analysis}

$\begin{array}{llll}\text { RM } & \text { Description } & \text { Temperature Range }\left({ }^{\circ} \mathbf{C}\right) & \text { Unit Size } \\ \text { GM } 754 & \text { ICTA Polystyrene DTA } & 97.8 \text { to } 107.5 & 10 \mathrm{~g} \\ 8759 & \text { ICTA Set DTA } & 295 \text { to } 675 & 5 \times 10 \mathrm{~g} \\ 8760 & \text { ICTA Set DTA } & 570 \text { to } 940 & 5 \times 10 \mathrm{~g}\end{array}$

Defining Fixed Points, International Temperature Scale of 1990, ITS-90
SRM
Description
Temperature $\left({ }^{\circ} \mathrm{C}\right)$
Unit Size (g)

Pure Metals

$\begin{array}{llll}743 & \text { Mercury (Triple Point) } & -38.8344 & \text { ampoule: } 680 \\ 1745 & \text { Indium (Freezing Point) } & 156.5985 & \text { ingot: } 20 \times 10 \mathrm{~g} \\ 741 \mathrm{a} & \text { Tin (Freezing Point) } & 231.928 & \text { shot: } 200 \\ 740 \mathrm{a} & \text { Zinc (Freezing Point) } & 419.527 & \text { shot: } 200 \\ 1744 & \text { Aluminum (Freezing Point) } & 660.323 & \text { ingot: } 200 \\ 1746 & \text { Silver (Freezing Point) } & 961.780 & \text { shot: } 300\end{array}$

Devices (semi-open cell)

$\begin{array}{lllr}1747 & \text { Tin (Freezing Point), 99.9999+\% } & 231.928 & 1071 \\ 1748 & \text { Zinc (Freezing Point), 99.9999+\% } & 419.527 & 1031\end{array}$




\section{Reference Points}

$\begin{array}{llll}\text { SRM } & \text { Description } & \text { Temperature }\left({ }^{\circ} \mathbf{C}\right) & \text { Unit Size (g) } \\ 742 & \text { Alumina, } 99.9+\% \text { (Melting Point) } & 2052 & \text { powder: } 10 \\ 45 d & \text { Copper (Freezing Point) } & 1084.6 & \text { bar: } 450 \\ 49 \mathrm{e} & \text { Lead (Freezing Point) } & 327.453 & \text { bar: } 600\end{array}$

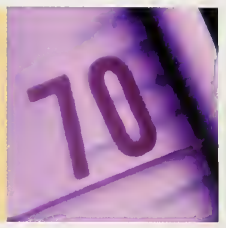

Freezing Point, Melting Point, and Triple Point Cells (sealed cell)

$\begin{array}{llll}\text { SRM } & \text { Description } & \text { Temperature }\left({ }^{\circ} \mathbf{C}\right) & \text { Unit Size (g) } \\ 1968 & \text { Gallium (Melting Point), 99.9999+\% } & 29.7646 & 25 \\ 1972 & \begin{array}{l}\text { 1,3-Dioxolan-2-one (Ethylene Carbonate) } \\ \text { (Triple Point), 99.999+\% }\end{array} & 36.3143 & 60 \\ 1969 & \text { Rubidium (Triple Point), 99.9+\% } & 39.30 & 154 \\ 1973 & \text { n-Docosane (Triple Point), 99.999+\% } & 43.879 & 60 \\ 1970 & \text { Succinonitrile (Triple Point), 99.999+\% } & 58.0642 & 60 \\ 1971 & \text { Indium (Freezing Point), 99.9999+\% } & 156.598 & 100\end{array}$

\section{Thermal Expansion of Metal and Glass}

$\begin{array}{llll}\text { SRM } & \text { Description } & \text { Temperature Range }(\mathbf{K}) & \text { Unit Size }(\mathbf{c m}) \\ \text { 731L1 } & \text { Borosilicate Glass } & 80 \text { to } 680 & 0.64 \times 5.1 \\ \text { 731L2 } & \text { Borosilicate Glass } & 80 \text { to } 680 & 0.64 \times 10.2 \\ \text { 731L3 } & \text { Borosilicate Glass } & 80 \text { to } 680 & 0.64 \times 15.2 \\ 736 \text { L1 } & \text { Copper } & 20 \text { to } 800 & 0.64 \times 5.1 \\ 738 & \text { AISI } 446 \text { Stainless Steel } & 293 \text { to } 780 & 0.64 \times 5.1\end{array}$

Thermal Resistance of Glass, Silica, and Polystyrene

$\begin{array}{ll}\text { SRM } & \text { Description } \\ 1453 & \text { Expanded Polystyrene Board } \\ 1450 \mathrm{c} & \text { Fibrous Glass Board } \\ 1449 & \text { Fumed Silica Board } \\ 1459 & \text { Fumed Silica Board }\end{array}$

$\begin{aligned} & \text { Thermal Resistance } \\ & \left(\mathbf{m}^{\mathbf{2}} \cdot \mathbf{K} \cdot \mathbf{W}^{\mathbf{1}}\right)\end{aligned}$
0.381 to 0.420
0.661 to 0.818
1.195 to 1.253
1.195 to 1.253

Unit Size $(\mathrm{cm})$

$66 \times 93 \times 1.34$

$61 \times 61 \times 2.54$

$60 \times 60 \times 2.54$

$30 \times 30 \times 2.54$ 


\section{Vapor Pressure of Metals}

$\begin{array}{lllll}\text { SRM } & \text { Description } & \begin{array}{l}\text { Pressure Range (Pa) } \\ \text { (K, ITS-90) }\end{array} & \text { Temperature Range } & \text { Unit Size } \\ 745 & \text { Gold } & 10^{3} \text { to } 10^{2} & 1300 \text { to } 2100 & \text { wire: } 0.14 \mathrm{~cm} \text { diameter } \times 15.2 \mathrm{~cm} \\ 746 & \text { Cadmium } & 10^{6} \text { to } 10^{1} & 350 \text { to } 594 & \text { rod: } 0.64 \mathrm{~cm} \text { diameter } \times 6.4 \mathrm{~cm}\end{array}$

Thermal Conductivity of Graphite and Iron

RM

Electrolytic Iron (2 K to $1000 \mathrm{~K}$ )

8420

8421

Graphite (5 K to $2500 K$ )

8424

8426
Conductivity Range $\left(\mathrm{W} \cdot \mathrm{m}^{-1} \cdot \mathrm{K}^{-1}\right)$

12.32 to 32.98

12.32 to 32.98

0.0354 to 32.96

0.0354 to 32.96
Unit Size

$0.64 \mathrm{~cm}$ diameter $\times 5.0 \mathrm{~cm}$

$3.17 \mathrm{~cm}$ diameter $\times 5.0 \mathrm{~cm}$

$0.64 \mathrm{~cm}$ diameter $\times 5.0 \mathrm{~cm}$

$2.54 \mathrm{~cm}$ diameter $\times 5.0 \mathrm{~cm}$

\section{Laboratory Thermometer (mercury in glass)}

Unit Size: 1 each

SRM Description
$934 \quad$ Clinical Laboratory Thermometer

\section{Thermocouple Material, Platinum}

Unit Size: 1 each

$\begin{array}{ll}\text { SRM } & \text { Description } \\ 1749 & \text { Gold vs. Platinum Thermocouple Thermometer } \\ 1967 & \text { Platinum Wire, High Purity (99.999+\%) } \\ 1750 & \text { Standard Platinum Resistance Thermometer }\end{array}$

Calibrated Points $\left({ }^{\circ} \mathrm{C}\right)$

-0.20 to +0.20 
OPTICAL PROPERTIES

Molecular Transmittance and Absorbance

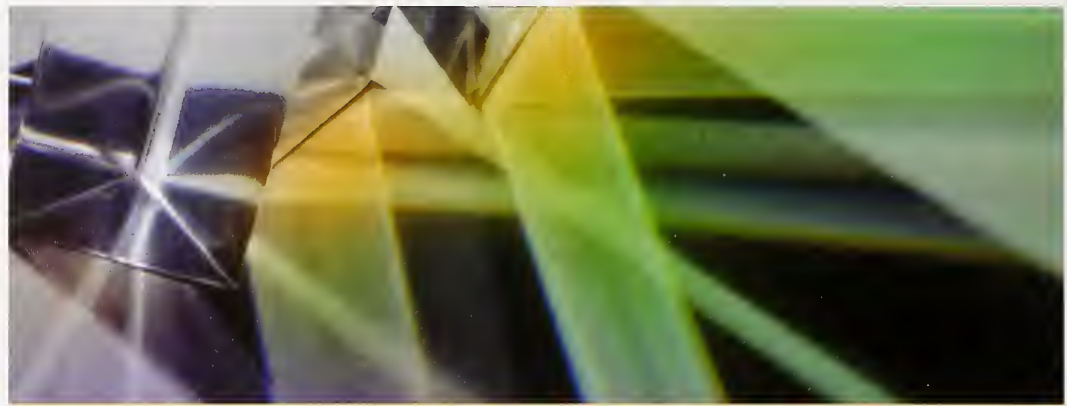

SRM Description

Crystalline and Solution Forms

935a Crystalline Potassium Dichromate, UV Absorbance

1935 Potassium Dichromate Solution, UV Absorbance

2032 Potassium lodide, Stray Light

931f Liquid Filters, Absorbance

Glass Filters, Transmittance

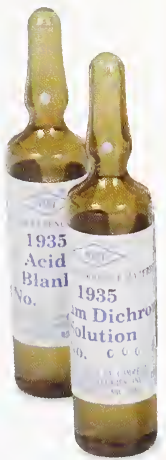

$930 \mathrm{e} \quad 10 \%, 20 \%, 30 \%$ Transmittance

$19301 \%, 3 \%, 50 \%$ Transmittance

2030a $30 \%$ Transmittance

2031a Metal-on-Quartz Filters $10 \%, 30 \%, 90 \%$ Transmittance

2046

2047

2048

2049

2050

2051

2053

2054

Optical Density $=1$

Optical Density $=2$

Optical Density $=3$

Optical Density $=4$

Optical Density $=5$

Optical Density $=6$

$20 \mathrm{~nm} \mathrm{Ni}-\mathrm{Cr}$ Film on Silica

2055

$90 \mathrm{~nm}$ Ni-Cr Film on Silica

2056
$77 \mathrm{~nm}$ Cu-Ni Film on Silica

$97 \mathrm{~nm}$ Cu-Ni Film on Silica

Wavelength Range

Unit Size

$235 \mathrm{~nm}$ to $350 \mathrm{~nm}$

$235 \mathrm{~nm}$ to $350 \mathrm{~nm}$

$240 \mathrm{~nm}$ to $275 \mathrm{~nm}$

$302 \mathrm{~nm}$ to $678 \mathrm{~nm}$

$440 \mathrm{~nm}$ to $635 \mathrm{~nm}$

$440 \mathrm{~nm}$ to $635 \mathrm{~nm}$

$465.0 \mathrm{~nm}$

$250 \mathrm{~nm}$ to $635 \mathrm{~nm}$

$1064 \mathrm{~nm}$

$1064 \mathrm{~nm}$

$1064 \mathrm{~nm}$

$1064 \mathrm{~nm}$

$1064 \mathrm{~nm}$

$1064 \mathrm{~nm}$

$2 \mu \mathrm{m}$ to $25 \mu \mathrm{m}$

$2 \mu \mathrm{m}$ to $25 \mu \mathrm{m}$

$2 \mu \mathrm{m}$ to $25 \mu \mathrm{m}$

$2 \mu \mathrm{m}$ to $20 \mu \mathrm{m}$
$15 g$

10 ampoules:

5 samples, plus 5 blanks

$25 \mathrm{~g}$

12 ampoules:

$3 \times 3$ levels, plus 3 blanks 


\section{Transmittance Wavelength Standards}

$\begin{array}{ll}\text { SRM } & \text { Description } \\ 2034 & \text { Holmium Oxide Solution } \\ 2035 & \text { Near IR Transmission } \\ 1921 a & \text { Infrared Transmission }\end{array}$

$\begin{array}{ll}\text { Wavelength Range } & \text { Unit Size } \\ 240 \mathrm{~nm} \text { to } 650 \mathrm{~nm} & 1 \text { sealed cuvette } \\ 971 \mathrm{~nm} \text { to } 1949 \mathrm{~nm} & 25 \mathrm{~mm} \text { diameter } \times 1.5 \mathrm{~mm} \\ 3.2 \mu \mathrm{m} \text { to } 18 \mu \mathrm{m} & 1 \text { polystyrene film }\end{array}$

\section{Fluorescence}

Description

Wavelength Range

Unit Size

936a Quinine Sulfate Dihydrate

$375 \mathrm{~nm}$ to to $675 \mathrm{~nm}$

$1 \mathrm{~g}$

\section{Specular Spectral Reflectance}

$\begin{array}{ll}\text { SRM } & \text { Description } \\ 2003 & \text { First Surface, Aluminum on Glass } \\ 2026 & \text { Second Surface, Aluminum on Fused Quartz } \\ 2040 & \text { PTFE Diffuser for Spectral Reflectance Factor }\end{array}$

Wavelength Range
$250 \mathrm{~nm}$ to $2500 \mathrm{~nm}$
$250 \mathrm{~nm}$ to $2500 \mathrm{~nm}$
$380 \mathrm{~nm}$ to $780 \mathrm{~nm}$

Unit Size

$5.1 \mathrm{~cm}$ diameter $\times 0.65 \mathrm{~cm}$

$5.1 \mathrm{~cm}$ diameter $\times 0.6 \mathrm{~cm}$ $5 \times 26 g$

\section{Near Infrared Reflectance Wavelength Standard}

$\begin{array}{ll}\text { SRM } & \text { Description } \\ \text { 1920a } & \text { Rare Earth Oxide Mixture }\end{array}$

\section{Wavelength Range}

$740 \mathrm{~nm}$ to $2000 \mathrm{~nm}$
Unit Size

$5.1 \mathrm{~cm}$ diameter $\times 1.2 \mathrm{~cm}$ 


\section{Optical Rotation}

$\begin{array}{llll}\text { SRM } & \text { Description } & \text { Wavelength Range } & \text { Unit Size } \\ 917 \mathrm{~b} & \text { D-Glucose (Dextrose) } & 546 \mathrm{~nm} \text { to } 589 \mathrm{~nm} & 50 \mathrm{~g} \\ 17 \mathrm{e} & \text { Sucrose } & 546 \mathrm{~nm} \text { to } 633 \mathrm{~nm} & 60 \mathrm{~g}\end{array}$

\section{Liquid Refractive Index}

$\begin{array}{llll}\text { SRM } & \text { Description } & \text { Wavelength Range } & \text { Unit Size } \\ 1922 & \text { Mineral Oil } & 468 \mathrm{~nm} \text { to } 589 \mathrm{~nm} & 30 \mathrm{~mL}\end{array}$

\section{X-ray and Photographic Imaging}

$\begin{array}{llc}\text { SRM } & \text { Description } & \text { Unit Size } \\ 1010 a & \text { Microcopy Resolution Test Chart } & 5 \text { charts } \\ 1008 & \text { Photographic Step Tablet } & 25.4 \mathrm{~cm} \times 3.5 \mathrm{~cm} \\ 1001 & \text { X-ray Film Step Tablet } & 25.4 \mathrm{~cm} \times 3.5 \mathrm{~cm}\end{array}$

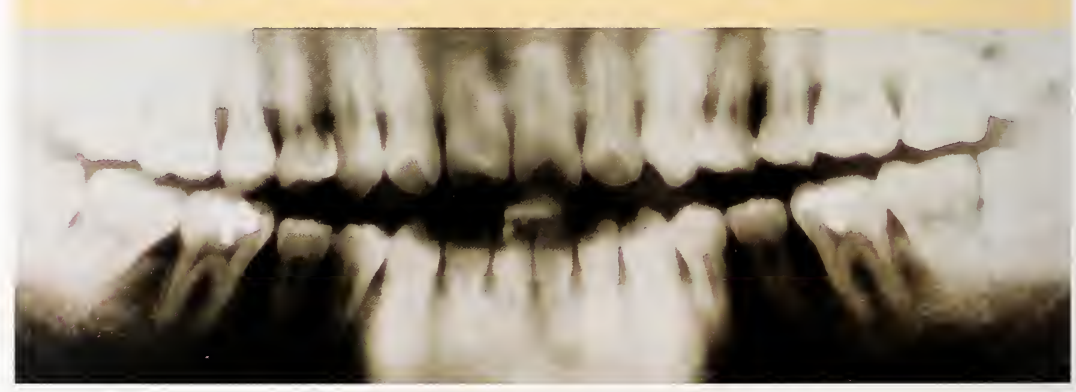




\section{ELECTRICAL PROPERTIES}

\section{Electrical Resistivity and Conductivity of Electrolytic Iron and Graphite}

Unit Size: rod: $0.64 \mathrm{~cm}$ diameter $\times 5.0 \mathrm{~cm}$

RM

Resistivity Range

Unit Size

$(\mu \Omega \cdot \mathrm{m})$

Electrolytic Iron (2 $K$ to $1000 K$ )
8420
0.004 to 0.909
$0.64 \mathrm{~cm}$ diameter $\times 5.0 \mathrm{~cm}$
8421
0.004 to 0.909
$3.17 \mathrm{~cm}$ diameter $\times 5.0 \mathrm{~cm}$

Graphite ( $5 K$ to $2500 K$ )

$\begin{array}{lll}8424 & 28.78 \text { to } 12.59 & 0.64 \mathrm{~cm} \text { diameter } \times 5.0 \mathrm{~cm} \\ 8426 & 28.78 \text { to } 12.59 & 2.54 \mathrm{~cm} \text { diameter } \times 5.0 \mathrm{~cm}\end{array}$

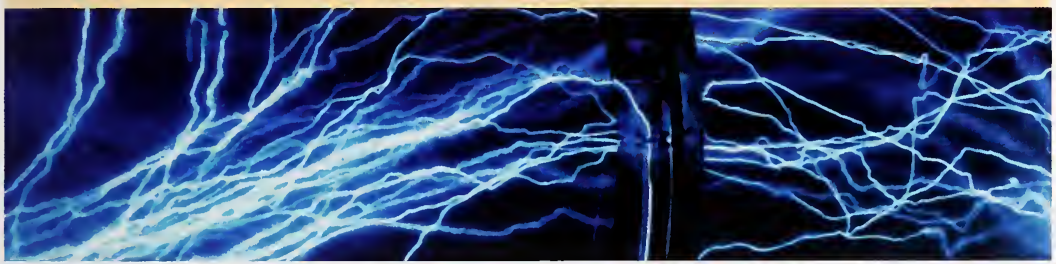

Electrical Resistivity and Conductivity of Silicon

SRM Resistivity $(\Omega \cdot \mathbf{c m})$

\section{Spreading Resistance}

Unit Size: 16 chips: $(5 \times 10 \times 0.625) \mathrm{mm}$

$2526 \quad 0.001$ to 200

$2527 \quad 0.001$ to 200

\section{Silicon Resistivity}

Unit Size: (100 diameter $\times 0.625) \mathrm{mm}$

$\begin{array}{ll}2544 & 10 \\ 2547 & 200\end{array}$

Type

\section{(111) p-type}

(111) n-type

float zone n-type silicon wafer with (III) crystallographic orientation 
OPTOELECTRONICS

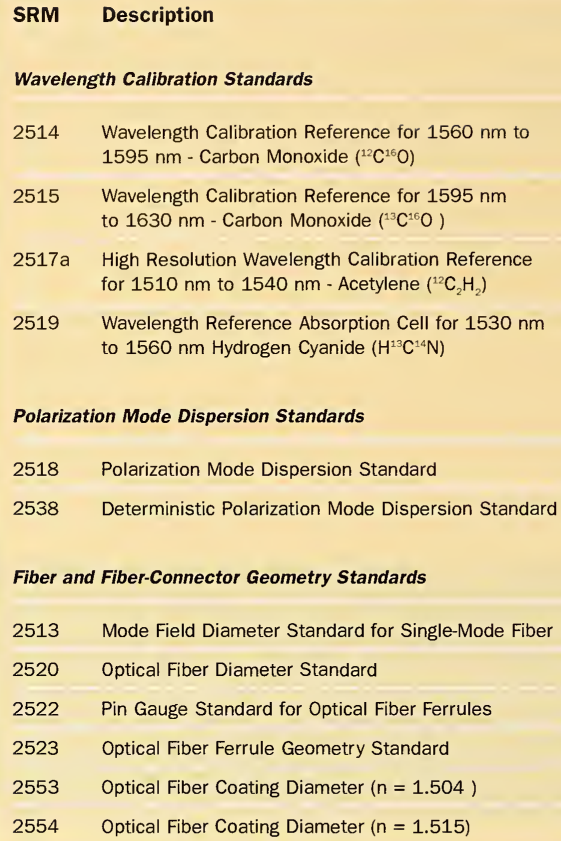

\section{Unit Size}

Gas Absorption Cell

Gas Absorption Cell

Gas Absorption Cell

Gas Absorption Cell

1 each

1 each

1 each

1 each

1 wire-sizing bore

1 ceramic connector ferrule

1 each: $250 \mu \mathrm{m}$ diameter

1 each: $250 \mu \mathrm{m}$ diameter

\section{E T R O L O G Y}

\section{Optical Microscope Linewidth Measurement}

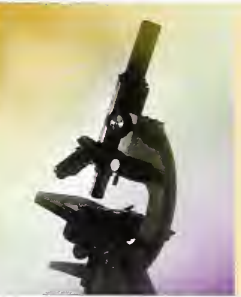

SRM Linewidth ( $\mu \mathrm{m})$

Pitch $(\mu \mathrm{m})$
Unit Size (cm)

\section{Linewidth Measurement Standards}

$\begin{array}{llll}475 & 0.9 \text { to } 10.8 & 2 \text { to } 36 & 6.35 \times 6.35 \times 0.15 \\ 476 & 0.9 \text { to } 10.8 & 2 \text { to } 36 & 6.35 \times 6.35 \times 0.15\end{array}$




\section{Scanning Electron Microscope (SEM)}

$\begin{array}{llll}\text { SRM } & \text { Description } & \text { Spacings } & \text { Unit Size }(\mathbf{m m}) \\ 2069 \mathrm{~b} & \text { SEM Performance Standard } & 2 \mathrm{~mm} \text { to } 4 \mathrm{~mm} & 12 \mathrm{~mm} \text { diameter with } 3 \mathrm{~mm} \text { peg } \\ 8091 & \text { SEM Sharpness Standard } & & \text { semiconductor chip: } 2 \mathrm{~mm} \times 2 \mathrm{~mm}\end{array}$

\section{Depth Profiling}

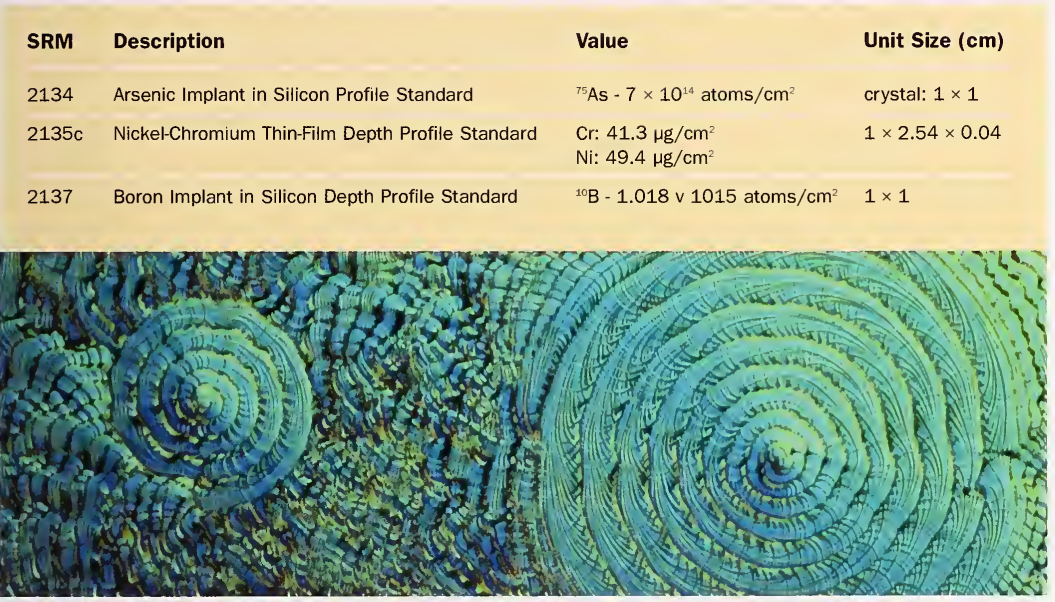

\section{Solder Thickness for X-ray Fluorescense}

Unit Size: plate: $15 \mathrm{~mm} \times 15 \mathrm{~mm}$

\begin{tabular}{|c|c|c|c|c|c|}
\hline \multirow[t]{2}{*}{ SRM } & \multirow[t]{2}{*}{ Description } & \multirow[t]{2}{*}{ Composition } & \multirow[t]{2}{*}{ Coating Mass/Area } & \multicolumn{2}{|c|}{ Coating Thickness } \\
\hline & & & & $\overline{(\mu \mathrm{m})}$ & $(\mu \mathrm{m})$ \\
\hline 2321 & Tin-Lead Alloy & $60 \% \mathrm{Sn}, 40 \% \mathrm{~Pb}$ & $6.8 \mathrm{mg} / \mathrm{cm}^{2}$ & 295 & 7.5 \\
\hline
\end{tabular}




\section{Coating Thickness}

Unit Size: $45 \mathrm{~mm} \times 45 \mathrm{~mm}$

These SRMs are suitable for calibrating instruments based on magnetic induction and magnetic pull-off techniques used in the measurement of organic and non-magnetic inorganic coatings over steel.

SRM Nominal Coating Thickness

( $\mu \mathrm{m}) \quad$ (mils)

Chromium over Copper on Steel

$\begin{array}{lll}1358 a & 80,255,1000 & 3.1,9.8,39 \\ 1359 b & 48,140,505,800 & 2.0,5.5,20,32 \\ 1361 b & 6,12,25,48 & 0.2,0.5,1.0,2.0 \\ 1362 b & 40,80,140,205 & 1.6,3.1,5.5,7.9 \\ 1363 b & 255,385,505,635 & 9.8,16,20,26 \\ 1364 b & 800,1000,1525,1935 & 32,39,59,79\end{array}$

\section{Ellipsometry}

Unit Size: $76 \mathrm{~mm}$ substrate diameter

Each unit is certified for the ellipsometric parameters delta $(\Delta)$ and psi $(\psi)$ at the vacuum wavelength $\lambda=633.0 \mathrm{~nm}$, and for the derived values of the thicknesses and indexes of refraction of the silicon dioxide and silicon layers.

SRM

Thin Film Thickness Standards

2531

2532

2533

2534

2535
Thickness (nm)

50

100

200

25

14 


\section{Oxygen Concentration in Silicon}

$\begin{array}{llll}\text { SRM } & \text { Description } & \text { Unit Size }(\mathbf{m m}) & \text { Concentration }(\mathbf{m g} / \mathbf{k g}) \\ 2551 & \text { Oxygen in Silicon } & 4 \text { wafers: } 25 \times 25 \times 2 & \text { Low: } 10 \\ & & \text { Medium: } 13 \\ & & \text { High: } 15 \\ & & \text { FZ: }(<0.1)\end{array}$

\section{Superconducting Critical Current (wire form)}

Unit Size: wire: $8.7 \mathrm{~cm}$ diameter $\times 2.2 \mathrm{~m}$

$\begin{array}{llll}\text { SRM } & \text { Description } & \text { Magnetic Field Range (T) } & \text { Critical Current Range (A) } \\ 1457 & \text { Niobium-Titanium Wire } & 2.000 \text { to } 8.000 & 293.30 \text { to } 69.72\end{array}$

\section{CERAMICSAND GLASSES}

\section{Chemical Resistance [Durability] of Glass}

$\begin{array}{lllll}\text { SRM } & \text { Description } & \mathbf{m L} \text { of } \mathbf{N} / \mathbf{5 0} \mathbf{H}_{\mathbf{2}} \mathbf{S O}_{\mathbf{4}} & \text { Unit Size (kg) } \\ 623 & \text { Borosilicate } & 0.34 & 2.2 \\ 622 & \text { Soda-Lime Silica } & 7.67 & 2.2\end{array}$

\section{Electrical Properties of Glass}

Unit Size: $5 \mathrm{~cm} \times 5 \mathrm{~cm} \times 2.5 \mathrm{~cm}$

SRM 624 is suitable for use with ASTM C 657. SRM 774 is suitable for use with ASTM D 150.

$\begin{array}{llll}\text { SRM } & \text { Description } & \text { Unit Size }(\mathbf{c m}) & \text { Value } \\ 624 & \text { Lead Silica for dc Volume Resistivity } & 5 \times 5 \times 2.5 & \log _{10} \rho \approx 9.9 \Omega \cdot \mathrm{cm} \text { at } 300^{\circ} \mathrm{C} \\ 774 & \begin{array}{l}\text { Lead Silica for Dielectric Constant } \\ \text { and ac Loss Characteristics }\end{array} & 5 \times 5 \times 2.5 & \mathrm{~K} \approx 7.47 \text { at } 100 \mathrm{~Hz}\end{array}$




\section{Viscosity of Glass}

$\begin{array}{ll}\text { SRM } & \text { Description } \\ 717 a & \text { Borosilicate Glass } \\ 710 a & \text { Soda-Lime-Silica Glass }\end{array}$

Unit Size $(\mathrm{mm})$

block: $40 \times 40 \times 150$

block: $100 \times 100 \times 40$

\section{Viscosity Fixpoints of Glass}

These SRMs are for the calibration of equipment for the determination of the softening, annealing, and strain points of glass.

$\begin{array}{ll}\text { SRM } & \text { Description } \\ 714 & \text { Alkaline Earth Alumina Silicate } \\ 717 a & \text { Borosilicate } \\ 713 & \text { Dense Barium Crown } 620 / 603 \text { Glass } \\ 709 & \text { Extra Dense Lead Silica } \\ 716 & \text { Neutral Glass } \\ 710 a & \text { Soda-Lime-Silica }\end{array}$

\section{Relative Stress Optical Coefficient}

\begin{tabular}{|c|c|c|}
\hline SRM & Description & $\begin{array}{l}\text { Relative Stress Optical Coefficient (C) } \\
\text { at } \lambda=546.1 \mathrm{~nm} \\
\left.\text { (Value } \times 10^{-12} \mathrm{~m}^{2} / \mathrm{N}\right)\end{array}$ \\
\hline 709 & Extra Dense Lead Silica & $C=-1.359$ \\
\hline
\end{tabular}

Unit Size

at $\lambda=546.1 \mathrm{~nm}$

at $\lambda=546.1 \mathrm{~nm}$
(Value $\times 10^{-12} \mathrm{~m}^{2} / \mathrm{N}$ )

709

Extra Dense Lead Silica

$C=-1.359$

\section{Density}

$\begin{array}{ll}\text { SRM } & \text { Description } \\ \text { 1827b } & \text { Lead Silica Glass } \\ \text { 211d } & \text { Toluene } \\ 2214 & \text { Isooctane }\end{array}$

\section{Density $\left(\mathrm{kg} / \mathrm{m}^{3}\right)$}

3593.800 at $20^{\circ} \mathrm{C}$

871.476 at $15^{\circ} \mathrm{C}$

695.969 at $15^{\circ} \mathrm{C}$

\section{Unit Size}

$225 \mathrm{~g}$

$40 \mathrm{~mm} \times 40 \mathrm{~mm} \times 150 \mathrm{~mm}$

$225 \mathrm{~g}$

$4 \mathrm{~cm} \times 4 \mathrm{~cm} \times 5 \mathrm{~cm}$

$250 \mathrm{~g}$

$100 \mathrm{~mm} \times 100 \mathrm{~mm} \times 40 \mathrm{~mm}$ 


\section{Glass Liquidus Temperature}

$\begin{array}{lllll}\text { SRM } & \text { Description } & \text { Unit Size } & \text { Method } & \text { Temperature }\left({ }^{\circ} \mathrm{C}\right) \\ 773 & \text { Soda-Lime-Silica } & 2.5 \mathrm{~cm} \times 2.5 \mathrm{~cm} \times 0.6 \mathrm{~cm} & \begin{array}{l}\text { A (boat) } \\ \text { B (perforated plate) }\end{array} & \begin{array}{l}988 \\ 991\end{array} \\ 1416 & \text { Aluminosilicate } & 22 \text { lengths of } 12.7 \mathrm{~cm} \text { tube }(250 \mathrm{~g}) & 1147\end{array}$

\section{X-RAY SPECTROMETRY}

\section{X-ray Diffraction}

\begin{tabular}{|c|c|c|c|}
\hline SRM & Description & XRD Application & Unit Size (g) \\
\hline 676 & Alumina (Corundum Structure) & Quantitative Analysis & 20 \\
\hline 1976 & Alumina Plate, Sintered & Instrument Response & $45 \mathrm{~mm} \times 45 \mathrm{~mm} \times 1.6 \mathrm{~mm}$ \\
\hline 2910 & Calcium Hydroxyapatite & Quantitative Analysis & 5 \\
\hline $660 a$ & Lanthanum Hexaboride Powder & Line Position, Line Shape & 6 \\
\hline 675 & Mica & Low $2 \theta$ (Large d-Spacing) & 7.5 \\
\hline $1879 a$ & Respirable Cristobalite & Quantitative Analysis & 5 \\
\hline $1878 a$ & Respirable Quartz & Quantitative Analysis & 5 \\
\hline 656 & Silicon Nitride & Quantitative Analysis & $2 \times 10 g$ \\
\hline $640 c$ & Silicon Powder 2 /d-Spacing & Line Position, Line Shape & 7.5 \\
\hline $674 b$ & $\begin{array}{l}\text { X-ray Powder Diffraction } \\
\text { Intensity Set }\left(\alpha-\mathrm{Al}_{2} \mathrm{O}_{3}, \mathrm{CeO}_{2} \text {, }\right. \\
\left.\mathrm{Cr}_{2} \mathrm{O}_{3}, \mathrm{TiO}_{2}, \mathrm{ZnO}\right) \text { (In Prep) }\end{array}$ & Quantitative Analysis & - \\
\hline
\end{tabular}

\section{X-ray Stage Calibration}

$\begin{array}{lll}\text { SRM } & \text { Description } & \text { Unit Size (mm) } \\ 1842 & \text { Calibration Board ( } \mathrm{X} \text { and } Y \text { dimensions) } & \text { Board: } 300 \times 300 \times 3 \\ 1843 & \text { Calibration Board }(Z \text { dimension }) & \text { Triangular Block: } 37 \times 20 \times 12\end{array}$


RADIOACTIVITY

85 Radioactive Solutions

87 Radioactive Point Sources

87 Radiopharmaceuticals

88 Radon Emanation

88 Beryillum Isotopic Ratio Standard

88 Carbon-14 Dating

89 Natural Matrix Materials

89 Neutron Density Monitor Wire

89 Fission Track Glass
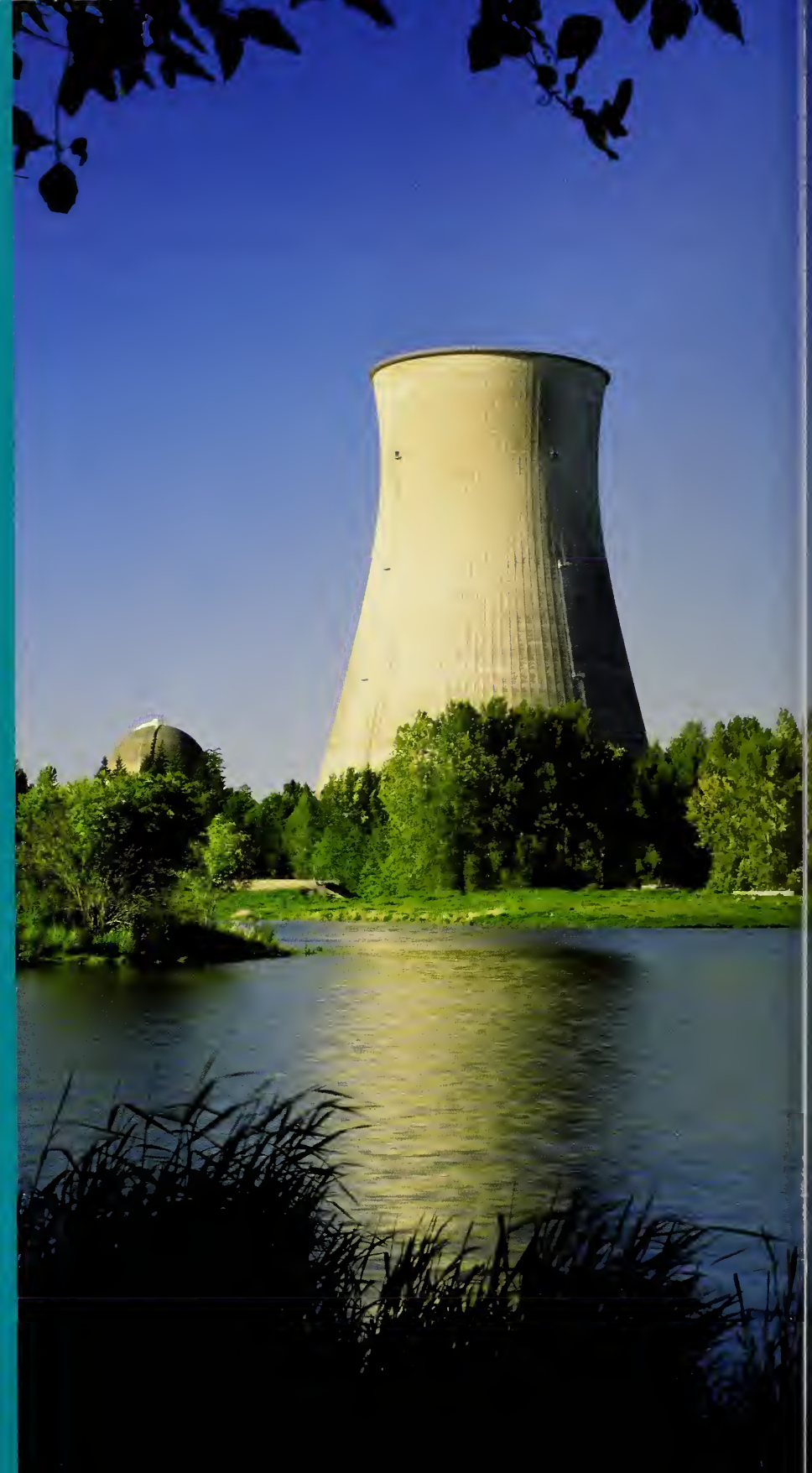


\section{Radioactive Solutions}

Each SRM is contained in a $5 \mathrm{~mL}$ flame-sealed glass ampoule and, except as noted, consists of the radionuclide dissolved in an aqueous solution (usually acidic, unless noted).

\begin{tabular}{|c|c|c|c|}
\hline SRM & Radionuclide & Massic Activity (Bq/g) & Calibration Date \\
\hline $4322 B *$ & Americium-241 & 40 & 09/1991 \\
\hline $4332 \mathrm{D} *$ & Americium-243 & 40 & 05/1995 \\
\hline $4251 C^{*}$ & Barium-133 & 500000 & $09 / 1993$ \\
\hline $4222 \mathrm{C}$ & Carbon-14 (as hexadecane) & 50000 & $09 / 1990$ \\
\hline 4233E* & Cesium-137 Burn-up Standard (In Prep) & 300000 & - \\
\hline 4943 & Chlorine-36 (3 mL) & 10000 & $12 / 1984$ \\
\hline $4915 E^{*}$ & Cobalt-60 & 75000 & 01/1995 \\
\hline $4329 *$ & Curium-243 & 70 & $06 / 1984$ \\
\hline $4320 A^{*}$ & Curium-244 & 35 & 02/1996 \\
\hline $4370 C *$ & Europium-152 & 90000 & $02 / 1987$ \\
\hline $4361 \mathrm{C}$ & Hydrogen-3 (as water) $(500 \mathrm{~mL})$ & 2 & $09 / 1998$ \\
\hline $4926 \mathrm{E}$ & Hydrogen-3 (as water) (20 mL) & 5000 & 09/1998 \\
\hline $4927 \mathrm{~F}$ & Hydrogen-3 (as water) & 600000 & 09/1998 \\
\hline $4947 C$ & Hydrogen-3 (as toluene) ( $4 \mathrm{~mL}$ ) & 300000 & 03/1987 \\
\hline $4949 C *$ & lodine-129 (dilute base solution) & 3000 & 03/1993 \\
\hline $4341 *$ & Neptunium-237 & 100 & 03/1992 \\
\hline $4226 C$ & Nickel-63 & 50000 & 08/1995 \\
\hline $4323 B^{*}$ & Plutonium-238 & 40 & $11 / 1999$ \\
\hline $4330 B^{*}$ & Plutonium-239 & 40 & $11 / 1999$ \\
\hline $4338 A^{*}$ & Plutonium-240 & 40 & 05/1996 \\
\hline $4340 B^{*}$ & Plutonium-241 (In Prep) & 250 & - \\
\hline $4334 \mathrm{G} *$ & Plutonium-242 & 25 & 06/1994 \\
\hline 4326 & Polonium-209 & 90 & 03/1994 \\
\hline
\end{tabular}

* These SRMs require a license certification.

(continued on next page) 
Radioactive Solutions (continued)

$\begin{array}{llll}\text { SRM } & \text { Radionuclide } & \text { Massic Activity } \mathbf{( B q} / \mathbf{g}) & \text { Calibration Date } \\ 4969 & \text { Radium-226 } & 3 & 09 / 1998 \\ 4965 & \text { Radium-226 } & 30 & 09 / 1991 \\ 4966 & \text { Radium-226 } & 270 & 09 / 1991 \\ 4967 & \text { Radium-226 } & 2700 & 09 / 1991 \\ 4339 B & \text { Radium-228 (In Prep) } & 500 & - \\ 4919 H^{*} & \text { Strontium-90 } & 4000 & 07 / 1995 \\ 4234 \text { A* } & \text { Strontium-90 } & 2500000 & 03 / 1995 \\ 4288 \text { A } & \text { Technetium-99 } & 30000 & 09 / 1996 \\ \text { 4328C } & \text { Thorium-229 (In Prep) } & 30 & - \\ \text { 4342A } & \text { Thorium-230 (In Prep) } & 40 & - \\ \text { 4324B } & \text { Uranium-232 (In Prep) } & 40 & 08 / 1997 \\ \text { 4321C } & \text { Uranium-238, Natural Uranium } & 250 & \end{array}$

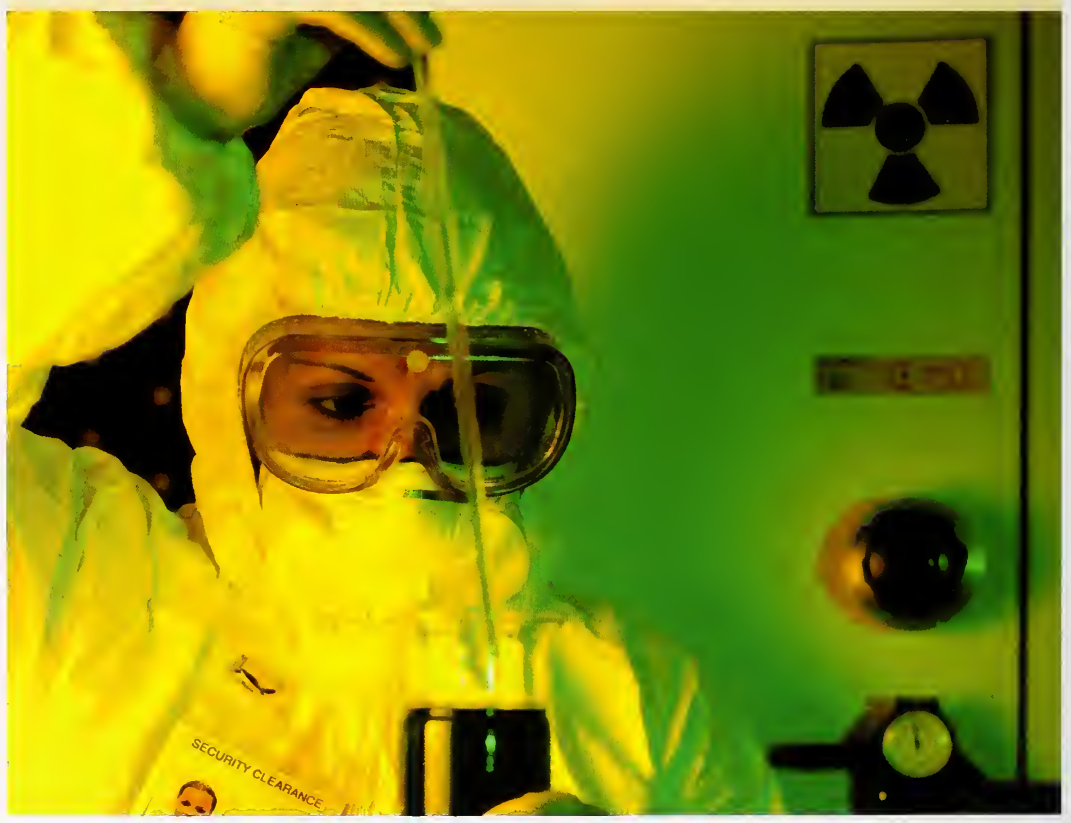

* These SRMs require a license certification. 


\section{Radioactive Point Sources}

$\begin{array}{lllll}\text { SRM } & \text { Radionuclide } & \begin{array}{l}\text { Principal Photon } \\ \text { Energies }(\mathbf{k e V})\end{array} & \begin{array}{l}\text { Activity } \\ \mathbf{( B q )}\end{array} & \begin{array}{l}\text { Calibration } \\ \text { Date }\end{array} \\ \text { 4241C } & \text { Barium-133 } & 81 \text { to } 384 & 60000 \text { to } 170000 & 01 / 1999 \\ \text { 4203D** Cobalt-60 } & 1173,1332 & 10000 \text { to } 60000 & 01 / 1995 \\ \text { 4218F* } & \text { Europium-152 } & 122 \text { to } 1400 & 50000 \text { to } 150000 & 01 / 1999 \\ \text { 4201B* } & \text { Niobium-94 } & 702,871 & 4000 & 04 / 1970\end{array}$

* These SRMs require a license certification.

\section{Radiopharmaceuticals}

\section{A typical schedule of SRMs for a year:}

$\begin{array}{lllll}\text { SRM } & \text { Radionuclide } & \text { High Level } & \text { Low Level } & \text { Month } \\ 4401^{*} & \text { lodine-131 } & 750 \mathrm{MBq}(20 \mathrm{mCi}) & 25 \mathrm{MBq}(700 \mu \mathrm{Ci}) & \text { January } \\ 4412^{*} & \text { Molybdenum-99 } & 1.5 \mathrm{GBq}(40 \mathrm{mCi}) & 75 \mathrm{MBq}(2 \mathrm{mCi}) & \text { February } \\ 4415^{*} & \text { Xenon-133 } & 7.5 \mathrm{GBq}(200 \mathrm{mCi}) & 750 \mathrm{MBq}(20 \mathrm{mCi}) & \text { March } \\ 4416^{*} & \text { Gallium-67 } & 375 \mathrm{MBq}(10 \mathrm{mCi}) & 20 \mathrm{MBq}(500 \mu \mathrm{Ci}) & \text { April } \\ & \text { OPEN } & & & \text { May } \\ 4404^{*} & \text { Thallium-201 } & 375 \mathrm{MBq}(10 \mathrm{mCi}) & 35 \mathrm{MBq}(900 \mu \mathrm{Ci}) & \text { June } \\ 442 *^{*} & \text { Samarium-153 } & 375 \mathrm{MBq}(10 \mathrm{mCi}) & 20 \mathrm{MBq}(500 \mu \mathrm{Ci}) & \text { July } \\ 4417^{*} & \text { Indium-111 } & 375 \mathrm{MBq}(10 \mathrm{mCi}) & 20 \mathrm{MBq}(500 \mu \mathrm{Ci}) & \text { August } \\ 4410^{*} & \text { Technetium-99m } & 7.5 \mathrm{GBq}(200 \mathrm{mCi})^{++} & ++ & \text {September } \\ 4407^{*} & \text { lodine-125 } & 750 \mathrm{MBq}(20 \mathrm{mCi}) & 6 \mathrm{MBq}(150 \mu \mathrm{Ci}) & \text { October } \\ & \text { OPEN } & & & \text { November } \\ 4427 * & \text { Yttrium-90 } & 200 \mathrm{MBq}(5 \mathrm{mCi}) & 20 \mathrm{MBq}(500 \mu \mathrm{Ci}) & \text { December }\end{array}$

* These SRMs require a license certification.

High level radiopharmaceutical SRMs are distributed through a program with the exception of technetium-99m. This program includes:

- Distribution of radioactivity Standard Reference Materials (usually a high level [ $10^{7} \mathrm{~Bq}$ to $10^{10} \mathrm{~Bq}$ ] and low level $\left[10^{6} \mathrm{~Bq}\right.$ to $10^{8} \mathrm{~Bq}$ ] pair of sources), for quantification, to sponsoring participating companies ten times per year.

- Two "open" months per year when the sponsors have the opportunity to send in sources of their choice for calibration.

For the more information, please contact Daniel Golas, the program's Project Manager, at NIST (301) 975-5540.

${ }^{\pi}$ Technetium-99m is also available to purchasers of low level radiopharmaceutical SRMs. 


\section{Radon Emanation Standard}

This SRM is intended for the calibration of radon-222 measuring instruments. It consists of a small heat-sealed polyethylene cylinder containing approximately $0.35 \mathrm{~g}$ of radium- 226 solution. The SRM is calibrated in terms of the radium- 226 activity and in terms of the emanation fraction of the radon- 222 under specified conditions.

$\begin{array}{llll}\text { SRM } & \text { Radionuclide } & \text { Activity }(\mathbf{B q}) & \text { Calibration Date } \\ 4968 & \text { Radium-226 } & 4,40 \text {, or } 400 & 09 / 1991\end{array}$

\section{Beryllium Isotopic Ratio Standard}

\begin{tabular}{llllll} 
SRM & Radionuclide & Isotopic Ratio & $\begin{array}{l}\text { Total Nuclide } \\
\text { Concentration } \\
\text { (mg/g) }\end{array}$ & $\begin{array}{l}\text { Calibration } \\
\text { Date }\end{array}$ & $\begin{array}{l}\text { Volume } \\
(\mathbf{m L})\end{array}$ \\
\hline 4325 & $3 \times 10^{-11}$ & 5 & $08 / 1986$ & 50 \\
\hline $\begin{array}{l}\text { Beryllium-10/ } \\
\text { Beryllium-9 } \\
\text { (in } 1 \mathrm{~N} \mathrm{HCl)}\end{array}$ & & & &
\end{tabular}

\section{Carbon-14 Dating Standard}

This SRM is an international standard for contemporary carbon-14 against which world-wide measurements can be compared.

SRI

Description

Unit Size

$4990 \mathrm{C} \quad$ Oxalic Acid

$8 \times 28 g$ 


\section{Natural Matrix Materials}

These SRMs are intended for use in testing measurements of low level, biological, and environmental radioactivity contained in matrices similar to the sample, for evaluating analytical methods, or as a generally available calibrated "real" sample matrix in interlaboratory comparisons.

$\begin{array}{lll}\text { SRM } & \text { Description } & \text { Unit Size (g) } \\ 4350 \mathrm{~B} & \text { Columbia River Sediment } & 85 \\ 4351 & \text { Human Lung } & 45 \\ 4352 & \text { Human Liver } & 45 \\ 4354 & \text { Freshwater Lake Sediment } & 25 \\ 4355 & \text { Peruvian Soil } & 75 \\ 4356 & \text { Ashed Bone } & 15 \\ 4357 & \text { Ocean Sediment } & 85\end{array}$

\section{Neutron Density Monitor Wire}

$\begin{array}{llll}\text { SRM } & \text { Description } & \begin{array}{l}\text { Cobalt Composition } \\ \text { (weight \%) }\end{array} & \text { Unit Size } \\ 953 & \text { Cobalt in Aluminum Wire } & 0.116 & 0.5 \mathrm{~mm} \text { diameter } \times 1 \mathrm{~m}\end{array}$

\section{Fission Track Glass}

Each unit consists of four unirradiated glass wafers and two irradiated wafers.

$\begin{array}{llllll}\text { SRM } & \begin{array}{l}\text { Uranium Composition } \\ (\boldsymbol{\mu g} / \mathbf{g})\end{array} & \begin{array}{l}\text { Uranium-235 } \\ (\text { Atom \%) }\end{array} & \begin{array}{l}\text { Reactor } \\ \text { Position }\end{array} & \begin{array}{l}\frac{\text { Neutron Fluence }}{\left(\times \mathbf{1 0}^{14} \mathbf{n} / \mathbf{c m}^{2}\right)} \\ \text { Copper Foil Gold Foil }\end{array} \\ 963 a & 0.823 & 0.2792 & \text { RT-4 } & 39.5 & 43.0 \\ & & & \text { RT-3 } & 41.2 & 45.8\end{array}$


INDUSTRIAL HYGIENE

91 Materials on Filter Media

91 Trace Constituent Elements in Blank Filters

91 Respirable Silica

92 Lead in Paint, Dust, and Soll 93 Asbestos 


\section{Materials on Filter Media}

These SRMs consist of potentially hazardous materials deposited on filters to be used to determine the levels of these materials in industrial atmospheres.

$\begin{array}{llllll}\text { SRM } & \text { Description } & \text { Set Size } & \begin{array}{l}\text { Elemental } \\ \text { Composition }\end{array} & \begin{array}{l}\text { Diameter } \\ (\mathbf{m m})\end{array} & \begin{array}{l}\text { Pore Size } \\ (\boldsymbol{\mu m})\end{array} \\ 2679 a & \text { Quartz on Filter Media } & \begin{array}{l}2 \times 3 \text { levels, } \\ \text { plus } 2 \text { blanks }\end{array} & \text { Quartz, Clay } & 47 & 0.45 \\ 2783 & \text { Air Particulate on Filter } & \begin{array}{l}2 \text { filters, } \\ \text { plus } 2 \text { blanks }\end{array} & \begin{array}{l}18 \text { certified values } \\ 9 \text { reference values }\end{array} & 47 & 0.4\end{array}$

\section{Trace Constituent Elements in Blank Filters}

SRMs 2678 and 2681 are for use in evaluating the performance of air sampling filter methods with either certified values (in $\mu \mathrm{g}$ ) or limits of detection $\left(\mathrm{X}_{\mathrm{p}}\right)$ for each of 30 constituent elements, as well as six leachable anions and cations.

$\begin{array}{lllll}\text { SRM } & \text { Description } & \text { Diameter }(\mathbf{m m}) & \text { Pore Size }(\boldsymbol{\mu m}) & \text { Filter Weight }(\mathbf{g}) \\ 2678 & \text { Cellulose Acetate Membrane } & 47 & 0.45 & 0.09 \\ 2681 & \text { Ashless Blank Filter } & 42.5 & - & 0.14\end{array}$

\section{Respirable Silica}

These SRMs are intended for use in determining, by X-ray diffraction, the levels of respirable silica in an industrial atmosphere according to the National Institute for Occupational Safety and Health (NIOSH) Analytical Method 7500 or equivalent methods.

$\begin{array}{llcc}\text { SRM } & \text { Description } & \text { Mass Fraction (\%) } & \text { Unit Size (g) } \\ 1878 a & \text { Respirable Alpha Quartz } & 100.00 \pm 0.21 & 5 \\ 1879 a & \text { Respirable Cristobalite } & 95.6 \pm 0.4 & 5\end{array}$




\section{Lead in Paint, Dust, and Soil}

These SRMs and RM have been developed in conjunction with the U.S. EPA to monitor paint, dust, and soil sources of lead.

SRM

Paint Film

2570

2571

2572

2573

2574

2575

2579a (Set of 6: SRMs 2570 to 2575)

2576 (High Level)

Powdered Paint

2580

2581

2582

2589

Indoor Dust, Trace Elements in (As, Cd, $\mathrm{Cr}, \mathrm{Hg}, \mathrm{Pb})$

2583

2584

Soil, Trace Elements in

2586

2587

Paint on Fiberboard

RM 8680
Lead Concentration

Unit Size

$<0.001 \mathrm{mg} / \mathrm{cm}^{2}$

$3.58 \mathrm{mg} / \mathrm{cm}^{2}$

$1.527 \mathrm{mg} / \mathrm{cm}^{2}$

$1.040 \mathrm{mg} / \mathrm{cm}^{2}$

$0.714 \mathrm{mg} / \mathrm{cm}^{2}$

$0.307 \mathrm{mg} / \mathrm{cm}^{2}$

0.307 to $3.58 \mathrm{mg} / \mathrm{cm}^{2}$

$5.59 \mathrm{mg} / \mathrm{cm}^{2}$

$4.34 \%$

$30 \mathrm{~g}$

$0.449 \%$

$35 \mathrm{~g}$

$209.8 \mathrm{mg} / \mathrm{kg}$

$20 \mathrm{~g}$

$9.99 \%$

$35 \mathrm{~g}$

1 film, plus 1 blank

1 film, plus 1 blank

1 film, plus 1 blank

1 film, plus 1 blank

1 film, plus 1 blank

5 films, plus 1 blank

1 film, plus 1 blank

$85.9 \mathrm{mg} / \mathrm{kg}$

$8 \mathrm{~g}$

$9761 \mathrm{mg} / \mathrm{kg}$

$8 \mathrm{~g}$

$432 \mathrm{mg} / \mathrm{kg}$

$50 \mathrm{~g}$

$3242 \mathrm{mg} / \mathrm{kg}$

$50 \mathrm{~g}$

1 to $2 \mathrm{mg} / \mathrm{cm}^{2}$

1 sheet:

$(10.2 \times 15.2 \times 1.3) \mathrm{cm}$ 


\section{Asbestos}

\begin{tabular}{|c|c|c|c|}
\hline SRM & Description & Asbestos Type & Unit Size \\
\hline $1866 a$ & Common Commercial Asbestos & $\begin{array}{l}\text { chrysotile } \\
\text { grunerite (Amosite) } \\
\text { riebeckite (Crocidolite) }\end{array}$ & $3 \times 4 g$ \\
\hline $1876 \mathrm{~b}$ & Chrysotile Asbestos for TEM & - & $\begin{array}{l}10 \text { sections: } \\
3 \mathrm{~mm} \times 3 \mathrm{~mm}\end{array}$ \\
\hline RM 8411 & Mixed Asbestos Research Filter & $\begin{array}{l}\text { chrysotile asbestos } \\
\text { grunerite (Amosite) }\end{array}$ & $1 \mathrm{~cm}^{2}$ \\
\hline
\end{tabular}

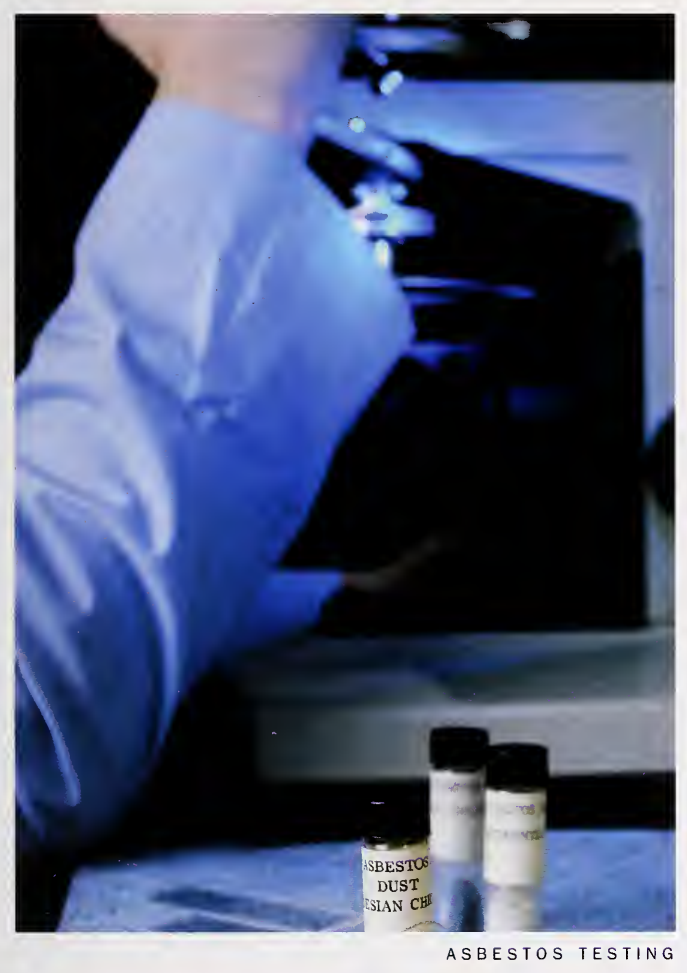


NIST develops and promotes measurements, standards, and technology to enhance productivity, facilitate trade, and improve the quality of life. As the U.S. National Metrology Institute, NIST continually strives to meet the nation's measurement needs with Standard Reference Materials, Calibration Services, and Standard Reference Data. Please visit our website at www.nist.gov for further information. 


\section{NGT}

National Institute of

Standards and Technology

Technology Administration

U.S. Department of Commerce 